\title{
WestVirginiaUniversity
}

THE RESEARCH REPOSITORY @ WVU

Graduate Theses, Dissertations, and Problem Reports

2015

\section{Comparisons of Single Drop Impact Simulations with Experiments}

Krishna Teja Medam

Follow this and additional works at: https://researchrepository.wvu.edu/etd

\section{Recommended Citation}

Medam, Krishna Teja, "Comparisons of Single Drop Impact Simulations with Experiments" (2015).

Graduate Theses, Dissertations, and Problem Reports. 6208.

https://researchrepository.wvu.edu/etd/6208

This Thesis is protected by copyright and/or related rights. It has been brought to you by the The Research Repository @ WVU with permission from the rights-holder(s). You are free to use this Thesis in any way that is permitted by the copyright and related rights legislation that applies to your use. For other uses you must obtain permission from the rights-holder(s) directly, unless additional rights are indicated by a Creative Commons license in the record and/ or on the work itself. This Thesis has been accepted for inclusion in WVU Graduate Theses, Dissertations, and Problem Reports collection by an authorized administrator of The Research Repository @ WVU. For more information, please contact researchrepository@mail.wvu.edu. 


\title{
Comparisons of Single Drop Impact Simulations with Experiments
}

By

\author{
Krishna Teja Medam
}

\author{
Thesis submitted to the \\ Benjamin M. Statler College of Engineering and Mineral Resources \\ at West Virginia University \\ in partial fulfillment of the requirements \\ for the degree of \\ Master of Science \\ in \\ Aerospace Engineering
}

Committee:

John M. Kuhlman, Ph.D., Chair

Donald D. Gray, Ph.D.

Christopher Griffin, Ph.D.

Department of Mechanical and Aerospace Engineering

Morgantown, West Virginia

2015

Keywords: Spray Cooling, VOF, CFD, ANSYS Fluent, OpenFOAM

Copyright 2015 Krishna Teja Medam 


\section{Abstract \\ Comparisons of Single Drop Impact Simulations with Experiments Krishna Teja Medam}

As the size of electronic equipment is reduced, the ability to reject waste heat is also reduced due to smaller component surface areas, thereby affecting the component performance and finally leading to the damage of the component. Spray cooling offers a means to achieve high rates of heat transfer from microelectronic components and other high energy density devices.

As a first step in investigating spray cooling, a single liquid drop impacting onto a thin liquid film was studied at isothermal conditions. 2D axisymmetric cases were simulated with ANSYS Fluent and 3D cases with OpenFOAM using the Volume of Fluid (VOF) model. The post processing of the results was performed in Surfer (Version 9) software in order to determine the liquid film thickness and then calculate the volume of the liquid under the cavity (sub-cavity liquid volume) as functions of time. These simulations agreed with the experimental data during the cavity formation phase, but did not closely match with the experiments during the refilling of the cavity in the majority of the cases. It was speculated that the discrepancies could be due to the three dimensional instabilities leading to droplet ejection from the crown during the retraction phase. These instabilities are omitted from the 2D simulations, and were not adequately resolved in the 3D simulations. For this reason, identical cases were simulated in 3D in OpenFOAM using the VOF model. The improved agreement with experiments obtained with the three dimensional simulations is discussed. 


\section{Table of Contents}

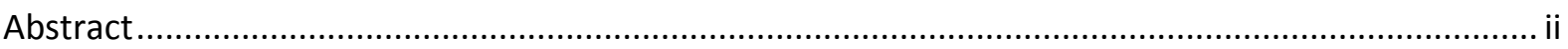

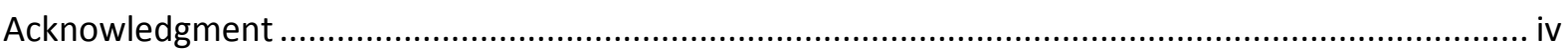

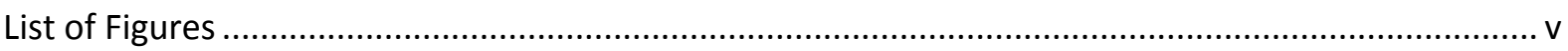

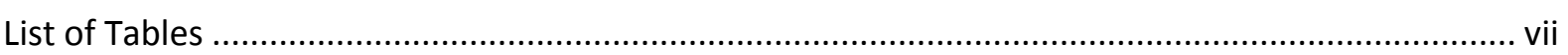

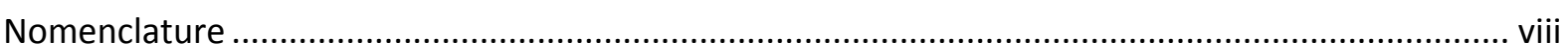

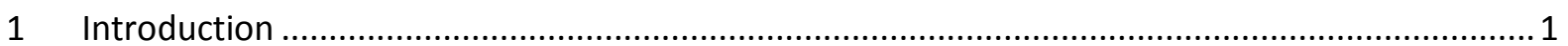

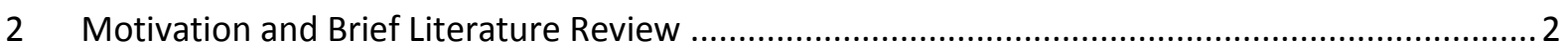

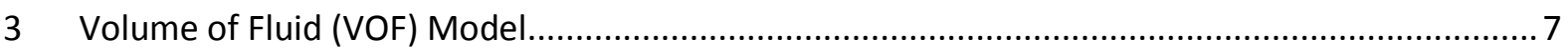

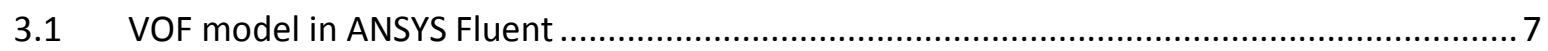

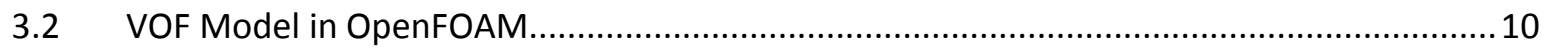

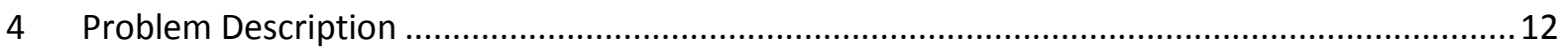

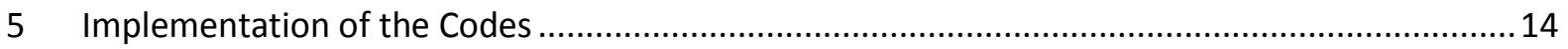

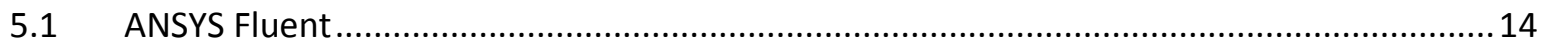

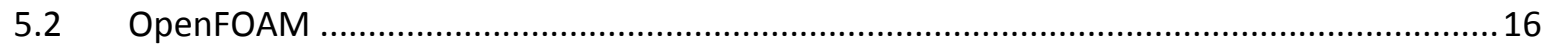

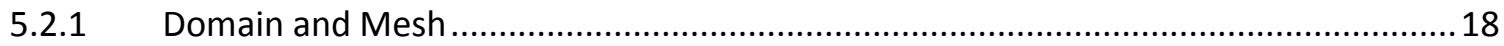

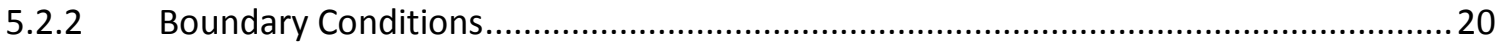

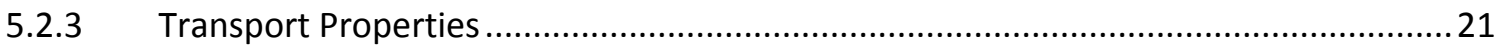

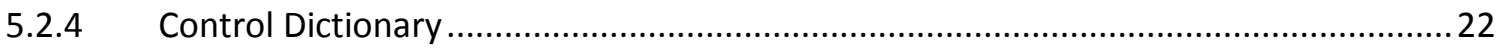

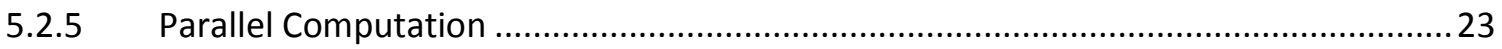

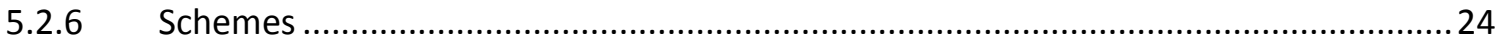

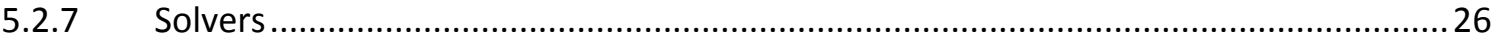

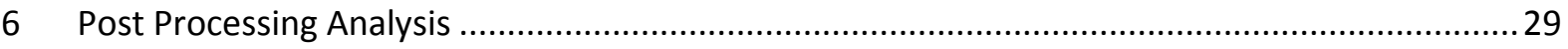

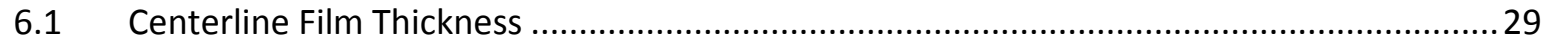

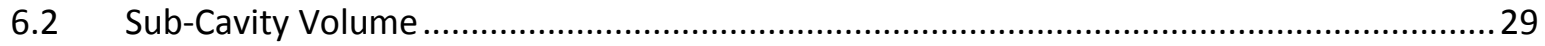

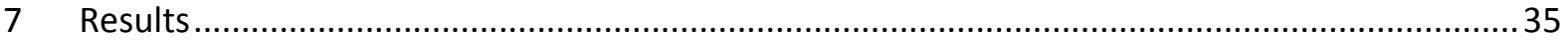

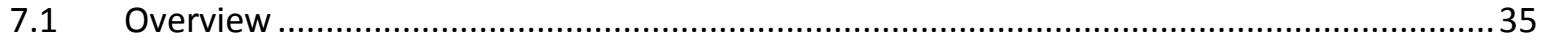

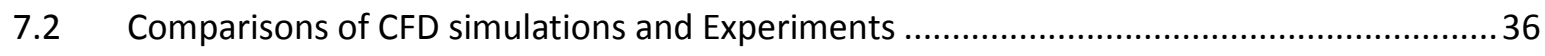

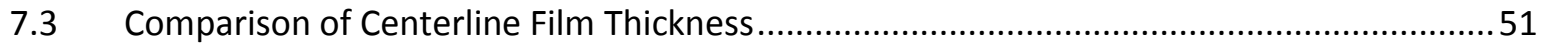

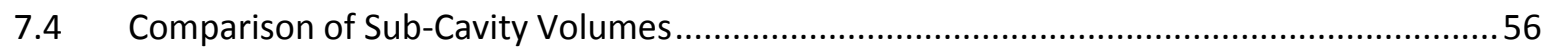

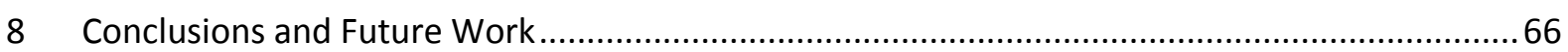

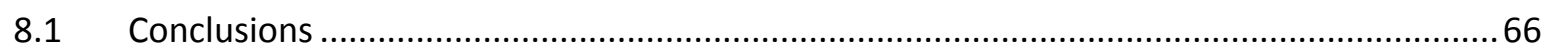

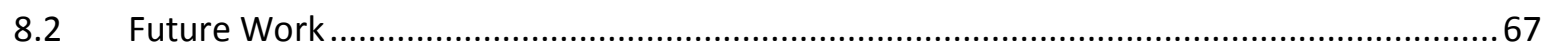

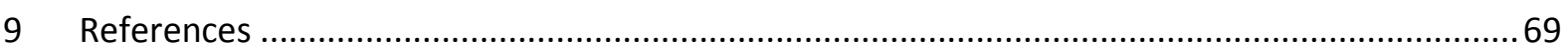




\section{Acknowledgment}

I first sincerely thank Dr. John M. Kuhlman and Dr. Donald D. Gray for recognizing me as their ideal candidate in completing the "Spray Cooling" project. Dr. Kuhlman guided me patiently even at his peak work load. I am really inspired by his time management, and was motivated to work on the same lines. I feel it's my great pleasure to work under him only to know that there is no end for learning.

I would like to thank Dr. Donald D. Gray for his patience in turning me into what I am now in research. He helped me to build up the way a researcher has to think in different ways to solve a problem. Without his constant feedback, I could have never known my mistakes and complete the tasks assigned to me. I would also like to thank Dr. Christopher Griffin, for his input in making my thesis a neat and complete work. During the initial stages of my work, he helped me to set up and get going with the simulations.

I also thank NASA for providing me an opportunity to work on their project and funding they provided to accomplish my Master's degree. I would like to specially thank National Science Foundation EPSCoR, the state of West Virginia (WVEPSCoR) and WVU for providing the crucial resource for accomplishing this work. Super Computing System (Mountaineer) is funded by them. I thank Mr. Nathan Gregg for his unmatched support and patience in answering my questions during the initial stages of running simulations in the cluster.

I cannot forget the support received from Mr. Martin Becker, developer of OpenFOAM, who helped me to solve the problems that I posted to him even during his busy schedule.

I thank my teammates, Mr. Murat Dinc, Mr. Stephen Taylor and Mr. Nicholas Hillen for their tremendous contribution in achieving the tasks. When I joined the project as naïve, Mr. Dinc spent his precious time in guiding me patiently and answering my questions. Mr. Taylor and Mr. Hillen suggested and helped me to think in a direction to approach the solution. I thank Mr. Sergio Escobar, Mr. Gennaro Campitelli, and Mr. Sai Satish Guda for helping me to solve many technical problems in setting up the simulations and working with Ansys Fluent and OpenFOAM.

I specially thank my family for their tremendous support and motivation in sending me this far to achieve my dreams even without any knowledge of what my research is. One of the most important person that I must be grateful is my would-be Kiruthika Krishnamoorthy. During the time I lost my focus and concentration, she would encourage me to get on track. I can never forget the times she helped me to push myself to the limits and bringing me out to know what I am capable of. 


\section{List of Figures}

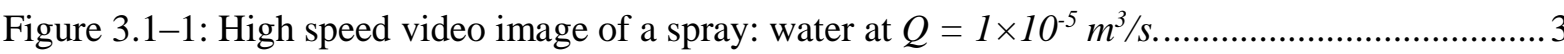

Figure 3.1-2: MC model simulation of FC-72 spray at different heater power levels with ................... 4

Figure 3.1-3: Worthington jet formed when a water drop impact on a deep pool of milk mixed with water (Yarin, 2006)

Figure 3.1-4: Drop impact pictured on an American postcard. Courtesy of Harold \& Ester Edgerton Foundation (2005) and Palm Press, Inc. (Yarin, 2006).

Figure 5.1-1: Boundary and initial conditions used in ANSYS Fluent 2D axisymmetric simulations. 15

Figure 5.2-1: Boundary conditions and domain for 3D OpenFOAM simulations............................. 17

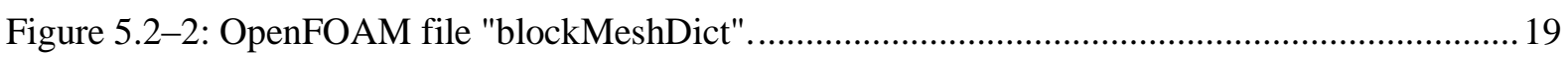

Figure 5.2-3: OpenFOAM mesh cropped and magnified to the same size as of Figure $5.1-1 \ldots \ldots \ldots . . .20$

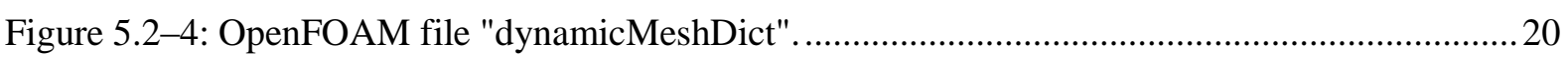

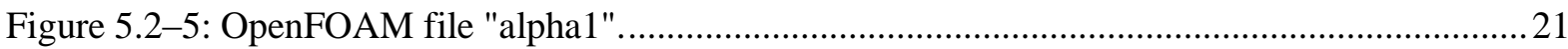

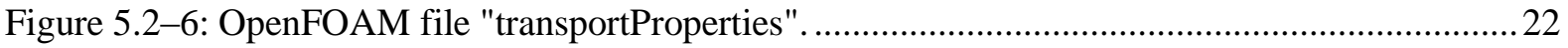

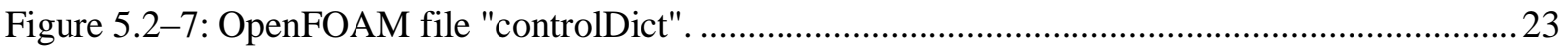

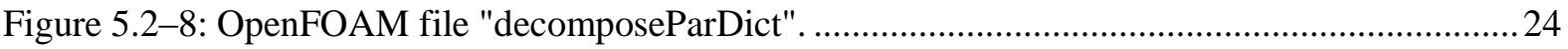

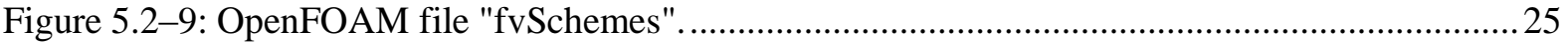

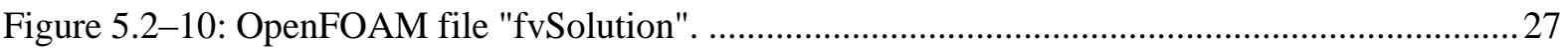

Figure 6.2-1: Example of the fillet at the outer radius of the liquid film thickness profile at ..............30

Figure 6.2-2: Radial location for outermost edge of sub-cavity region, highlighted in yellow, using cutoff value equal to 1.3 times the average centerline $\mathrm{h}$ at $\mathrm{t}=20 \mathrm{~ms}$; We $=141, \operatorname{Re}=5700, \mathrm{~h} / \mathrm{d}=$ 0.614

Figure 6.2-3: Computed sub-cavity liquid volumes vs. time, for a range of liquid film thickness cutoff values; $\mathrm{We}=141, \mathrm{Re}=5700, \mathrm{~h} / \mathrm{d}=0.614$

Figure 6.2-4: Sub-cavity liquid film thickness radial profile at $20 \mathrm{~ms}$ for $1.6 * \mathrm{~h} \_\mathrm{min}$, and horizontal axis is set at dimensional cutoff thickness value of $0.1302736 \mathrm{~mm}$; $\mathrm{We}=141, \mathrm{Re}=5700, \mathrm{~h} / \mathrm{d}=0.614$.

Figure 6.2-5: Sub-cavity liquid film thickness radial profile at $25 \mathrm{~ms}$ for $1.6 * \mathrm{~h} \_\mathrm{min}$, and horizontal axis is set at dimensional cutoff thickness value of $0.1302736 \mathrm{~mm}$; We $=141, \quad \operatorname{Re}=5700, \mathrm{~h} / \mathrm{d}=$ 0.614

Figure 7.2-1: Sequence of images for the case $1 \mathrm{~A}(\mathrm{We}=145, \mathrm{Re}=1189, \mathrm{~h} / \mathrm{d}=1.0)$ : First column is 2D Axisymmetric Fluent, Second column is 3D OpenFOAM and Third column is Experiment. ........ 37 Figure 7.2-2: Representation of the perspective view while capturing the videos for the experiments performed by Hillen in Hillen, 2013.

Figure 7.2-3: Sequence of images for the case $1 \mathrm{C}(\mathrm{We}=135, \mathrm{Re}=1176, \mathrm{~h} / \mathrm{d}=0.2)$ : First column is 2D Axisymmetric Fluent, Second column is 3D OpenFOAM and Third column is Experiment. 
Figure 7.2-4: Zoomed sequence of images for 2D axisymmetric Fluent simulation of case 1C. 40

Figure 7.2-5: Zoomed sequence of images for 3D OpenFOAM simulation of case 1C

Figure 7.2-6: Zoomed sequence of images for experiment of case 1C (Hillen, 2013) .42

Figure 7.2-7: Sequence of images for the case $3 \mathrm{~B}(\mathrm{We}=621, \mathrm{Re}=2643$, and $\mathrm{h} / \mathrm{d}=0.5)$ : First column is 2D Axisymmetric Fluent, Second column is 3D OpenFOAM and third column is Experiment. ......44 Figure 7.2-8: Sequence of images for the case $5 \mathrm{~A}(\mathrm{We}=993, \mathrm{Re}=3574$, and $\mathrm{h} / \mathrm{d}=0.9)$ : First column is 2D Axisymmetric Fluent, Second column is 3D OpenFOAM and third column is Experiment. .....45 Figure 7.2-9: Sequence of images for the case $5 \mathrm{C}(\mathrm{We}=984, \mathrm{Re}=3555$, and $\mathrm{h} / \mathrm{d}=0.2)$ : First column is 2D Axisymmetric Fluent, Second column is 3D OpenFOAM and third column is Experiment. ..... 46 Figure 7.2-10: Zoomed sequence of images for 2D axisymmetric Fluent simulation of case 5C. ...... 48 Figure 7.2-11: Zoomed sequence of images for 3D OpenFOAM simulation of case 5C. ..................49

Figure 7.2-12: Zoomed sequence of images for experiment of case 5C (Hillen, 2013)......................50

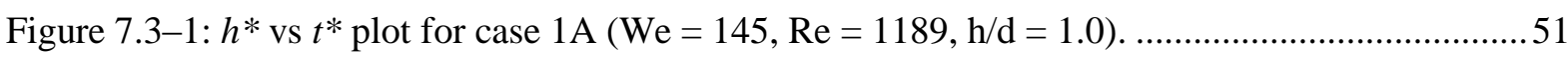

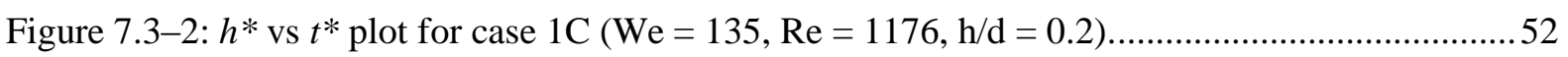

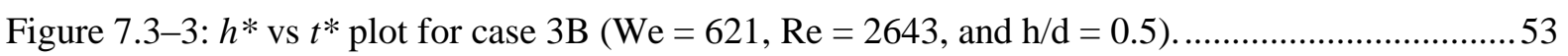

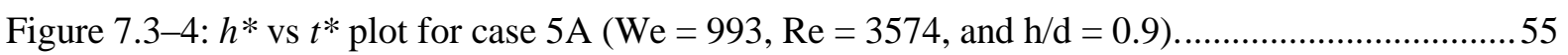

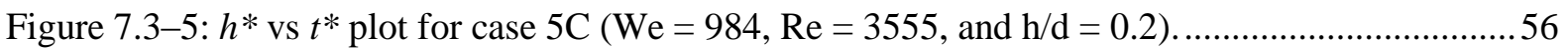

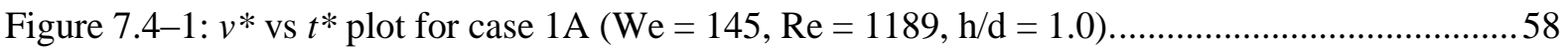

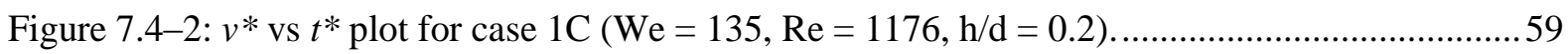

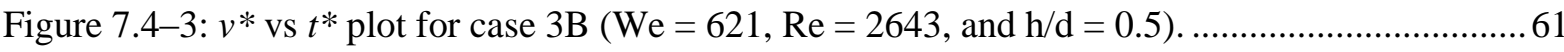

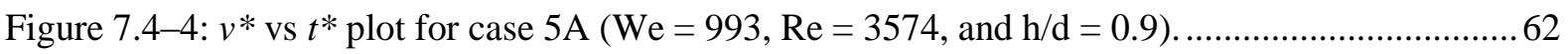

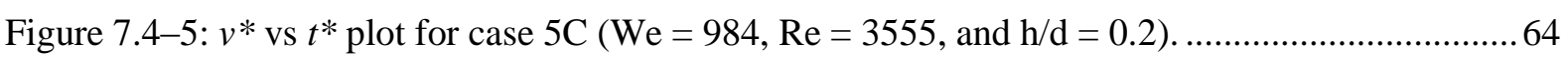




\section{List of Tables}

Table 4-1: Dimensional and non-dimensional parameters of simulated single drop cases; from experiments by Hillen (2013). .

Table 5-1: Properties of the liquid and air used in ANSYS Fluent. .14

Table 6-1: Cut-off heights used for experiments and CFD simulations...... .34

Table 7-1: Percentage deviation of sub-cavity volume for all the cases.............................................65

Table 7-2: Percentage of drop liquid volume present in the sub-cavity volume... .65 


\section{Nomenclature}

CFD: $\quad$ Computational Fluid Dynamics

MC Model: $\quad$ Monte-Carlo Model

VOF: $\quad$ Volume of Fluids

Q: $\quad$ Volume flow rate

2D: $\quad$ Two Dimensional

3D: $\quad$ Three Dimensional

We: $\quad$ Weber Number

Re: $\quad$ Reynolds Number

Fr: $\quad$ Froude Number

Oh: $\quad$ Ohnesorge Number

h: $\quad$ Height of the liquid above the bottom wall at the impact center

$h_{0}: \quad$ Initial static liquid layer height above the bottom wall

$h_{0}{ }^{*}: \quad \quad$ Normalized static liquid layer height $\left(h_{0} / d\right)$

d: $\quad$ Diameter of the impinging droplet

$\mathrm{h}^{*}$ : $\quad$ Normalized height of the liquid $(\mathrm{h} / \mathrm{d})$

Vel: $\quad$ Velocity of the impinging droplet

$\mathrm{t}^{*}: \quad=t \frac{V e l}{d}$

$V_{\text {drop }}: \quad$ Drop volume

$V_{\text {sub-cavity: }}$ Sub-cavity volume

$V^{*}: \quad=V_{\text {sub-cavity }} / V_{\text {drop }}$

h_min: $\quad$ Average height of the liquid at the impact center over time

CHF: $\quad$ Critical Heat Flux

CSF: $\quad$ Continuum Surface Force model

CSS: $\quad$ Continuum Surface Stress model

GUI: $\quad$ Graphical User Interface

RAM: $\quad$ Random Access Memory

EPSCoR: Experimental Program to Stimulate Competitive Research

WVEPSCoR: West Virginia Experimental Program to Stimulate Competitive Research 


\section{Introduction}

With the growing levels of technology in the present world, the size of the electronic components continue to reduce from generation to generation. Heat is produced by these components as the byproduct of their output. When this heat is not rejected or controlled properly, it may lead to a rise in temperature of the components and eventually cause the device to fail. Smaller sizes of electronics leads to a smaller surface area across which to reject waste heat, this requires higher heat fluxes. Spray cooling is a method to control a device's temperature, and remove the heat emitted from the components. When a liquid at normal room temperature is sprayed onto the electronic components or onto the heated surfaces, the liquid heats up due to conduction, reaching its boiling temperature, and eventually evaporates, thus transferring the waste heat from the surface. Simulating the spray and its interaction with the hot surface requires very high computational power. As a beginning step in the process of studying the heat transfer from the surface, a single drop is extracted from the spray, and is analyzed herein using Computational Fluid Dynamics (CFD). Based on the expected range of spray droplet Reynolds number, Weber number, and Froude number. This thesis compares the isothermal single drop simulations, in both full 3D and in 2D axisymmetric cases, to experimental results from the thesis by Hillen (2013), and discusses the discrepancies that arise in the calculation of centerline film thickness and sub-cavity volumes. These discrepancies are possibly due to the variation in surface tension of the liquid in the experiments, as well as limits in grid resolution due to limited computational resources for the CFD.

Isothermal cases were simulated as 2D axisymmetric using the ANSYS Fluent 14.5 code, while 3D simulations were performed using OpenFOAM 2.2.2 (Jasak et al., 2007). To be consistent with the simulations, both 2D axisymmetric and 3D simulations were simulated with the Volume of Fluid (VOF) computational model. This model gives better interface capture relative to the level set method, as the cases simulated here require precise liquid thickness height in order to calculate the sub-cavity liquid volume. This sub-cavity volume is required for calculation of the amount of heat required to evaporate the sub-cavity volume of liquid, and thus the heat transfer that is achieved from the heated surface. The present work is an extension of the single droplet studies described in the project final report by Jaridi et al. (2014). 


\section{Motivation and Brief Literature Review}

Spray Cooling is an effective method to cool heated surfaces. Due to today's rapid growth of technology, the size of the electronics has been reduced, resulting in the decrease of the surface area. The decrease in this area increases the required heat flux, which poses a great problem to the respective electronic components in terms of heat rejection. When the component is active it generates heat, causing the temperature of the component to rise. When the temperature is not adequately controlled, it may lead to the failure of the component. This is where spray cooling helps to reduce the component temperature. When a cool liquid is sprayed on to the hot surface of the component, the liquid is accumulated over the surface and starts to heat up as it absorbs heat from the component. The accumulated liquid layer quickly reaches a certain thickness, and any further spraying of liquid creates cavities in the liquid layer, due to individual droplet impacts. This impact of cool liquid drops on to the residual liquid layer results in heat transfer with high heat flux regions in the thin films beneath each impact cavity. These cavities have a very thin layer of liquid where the liquid heats up quickly and soon starts boiling. These cavity sites become initial active locations for nucleate boiling (Campbell et al., 1999) and eventually the liquid evaporates. When the liquid has evaporated, there is a reduction in heat transfer from the surface in the cavity. A heat transfer mechanism takes place where the liquid is heated, boiled and turned into vapor. Once the cavities dry out, they could contribute to the onset of critical heat flux.

This process of cooling is very effective for applications in space technology, defense, food industry, and fuel sprays in gas turbines, compared to the standard air-cooled systems. The high heat fluxes in spray cooling can keep the electronics in the required operating temperature range. This helps in avoiding any failures due to elevated component temperatures. Heat flux reaching up to $700 \mathrm{~W} / \mathrm{cm}^{2}$ was claimed to be possible at: NSA at Supercomputing Research (2009). Using a micromachined spray head cooling heat ejection of $250 \mathrm{~W} / \mathrm{cm}^{2}$ was achieved.

In order to predict any behavior associated with the sprays, the spray cooling process needs to be numerically modelled, and the numerical model must initially be validated with existing experiments. It is extremely difficult to completely model the sprays through CFD due to their complexity. It 
requires huge resources to successfully simulate the sprays even with modern meshing methods and numerical models. Kreitzer (2010) and Kuhlman et al. (2011) state that the droplet impacts in a dense spray may reach fluxes exceeding $10^{6} \mathrm{drops} /\left(\mathrm{s}-\mathrm{cm}^{2}\right)$. These types of sprays pose difficulties in simulating. Figure 3.1-1 shows an example high speed video image of the spray with the volume flow rate, $Q=1 \times 10^{-5} \mathrm{~m}^{3} / \mathrm{s}$ taken from their spray experiments. These images were used in measuring the cavity formation and its lifetime.

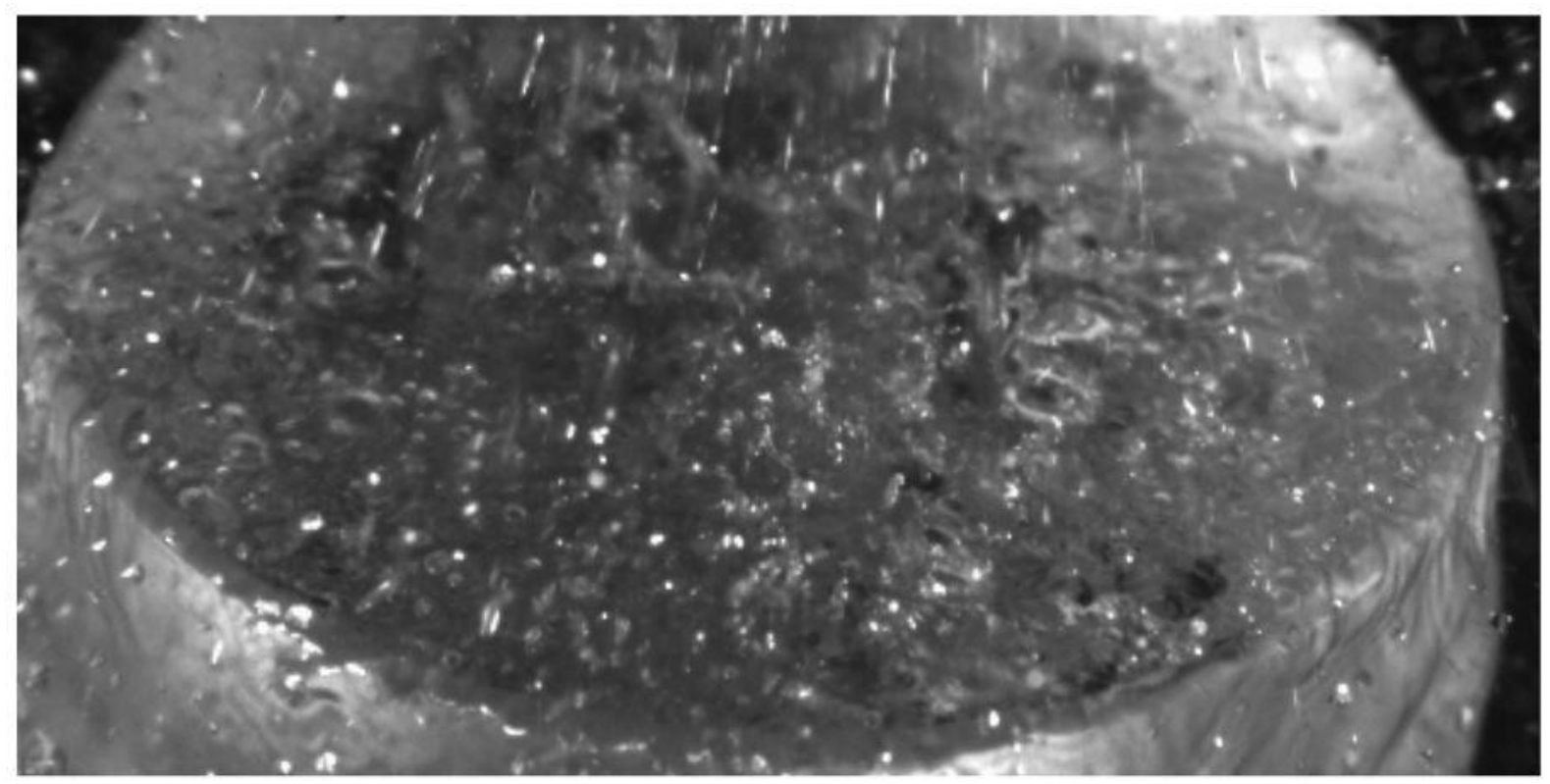

Figure 3.1-1: High speed video image of a spray: water at $Q=1 \times 10^{-5} \mathrm{~m}^{3} / \mathrm{s}$.

To develop a practical model of a spray a Monte-Carlo (MC) simulation model was developed to predict the spray characteristics similar to the experiments conducted by Kreitzer (Kreitzer, 2010). Using the MC model, the simulation was performed for varying heater power for the cooling liquid, FC-72. The heater power levels range from $60 \mathrm{~W}$ to $140 \mathrm{~W}$. These simulations predicted the same trend that is observed in the experiments regarding the onset of boiling and dry out regions near the outer edge of the heater surface. Figure 3.1-2 shows the regions on the heater surface in different colors depending on the temperature. The boiling regions are in orange and dry out regions colored in red. The dry out regions are concentrated near the outer edge of the heater surface while blue represents the original liquid layer and recent drop impact cavities with subcooled liquid. 


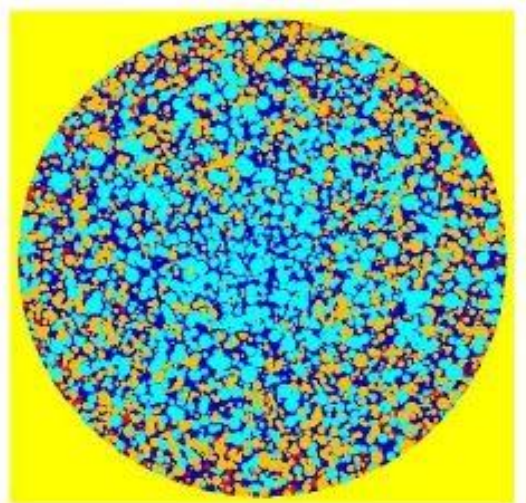

$60 \mathrm{~W}$

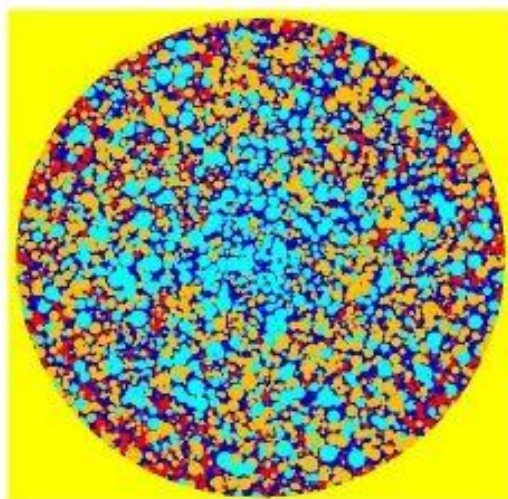

$100 \mathrm{~W}$

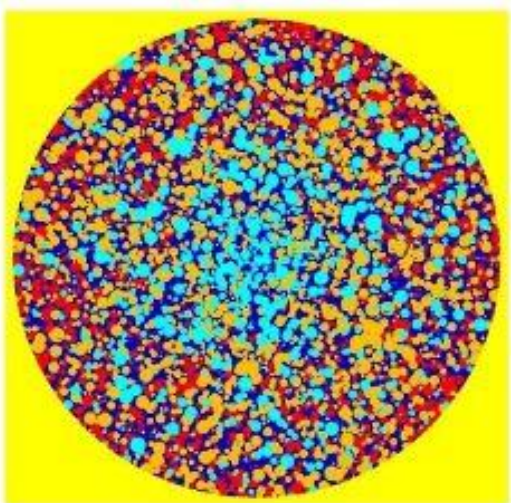

$140 \mathrm{~W}$

Figure 3.1-2: MC model simulation of FC-72 spray at different heater power levels with $Q=1 \times 10^{-5} \mathrm{~m}^{3} / \mathrm{s}$.

There are several factors that increase the complexity of the research on spray cooling. It depends on spray parameters such as: heater surface characteristics, geometry and roughness, ambient atmospheric conditions, spraying liquid, and gravity (Silk et al., 2008). When the droplets impinge on the heated surface covered with residual liquid film, they form cavities and secondary splashing occurs. Kuhlman et al. (2011), assumed that for this secondary splashing, $25 \%$ of the drop volume is splashed. Also, the thin liquid film that remains under the cavity was termed as the "sub-cavity region", and the volume of liquid was termed as the "sub-cavity volume". Due to effects of the surface tension, impact of neighboring droplets, or effect of gravity, the cavity fills in quickly, and can form a "Worthington jet" that rises from the surface to a maximum height that depends on the velocity of drop at the time of impact, gravity and surface tension.

In order to determine the percentage of drop liquid and the percentage of film liquid in the sub-cavity volume, first the interface between the drop liquid and film liquid has to be precisely tracked, and then the calculation of the volume of the drop liquid is possible. Since it is expected that the film liquid would be hotter than the drop liquid in a spray cooling application, the percentage of the sub-cavity volume that comes from the original layer fluid will influence the amount of sensible heating that is required in order to initiate boiling of the sub-cavity liquid. The initial work to track the drop liquidfilm liquid interface was performed by Josserand in Josserand et al. 2003. For a constant We of 8000, they varied Re from 1000 to 40. Josserand observed the breaking of crown liquid depending on the viscosity and Re. For low viscous fluids splashing is seen. Conversely, when the Re is low, the drop liquid is gently deposited on the inner side of the cavity without any splashing. 
In these simulations, the interface between the drop liquid and the film liquid is distorted due to the entrapment of air at the time of impact, as explained by Josserand in Josserand et al. 2010. By creating a high resolution, adaptive mesh with levels varying from 8 to 12 (mesh size of $2 \mu \mathrm{m}$ to $8 \mu \mathrm{m}$ ), the air trapped in between the two liquid layers is resolved in these more recent simulations, and a dimple and gas bubbles are formed at the interface. This is due to the creation of high pressure regions formed by the viscosity of the gas. Sub-cavity volume percentages of drop liquid and film liquid were calculated and presented in Kuhlman et al. 2014. In this paper, the simulations were done with a mesh resolution of level 3 and level 4 . Level 3 corresponds to a minimum mesh size of $d / 42$ and level 4 is of $d / 84$, where $d$ is the diameter of the drop. For high Re values, but with We values similar to those used in this thesis, the percent of drop liquid volume and percent film liquid volume is separately calculated in order to understand the percentage of drop liquid present in the sub-cavity volume.

The present thesis discusses the impact of single drops on to a static liquid film, with varying $h / d$ ratio and Weber number. Here the $h_{0} / d$ ratio is equivalent to $h^{*}$ - the non-dimensional height, which is equal to the ratio of static liquid film thickness $\left(h_{0}\right)$ to the drop diameter $(d)$. In the investigation of spray cooling, the central aim is to accurately predict the amount of heat transfer that is taking place, and to calculate the Critical Heat Flux (CHF). Yarin (2006), has documented the details of individual drop impact, crown formation, criteria for secondary splashing, Worthington jet formation (Figure 3.1-3), and the effects of drop impact on both thin liquid films and on dry surfaces. Figure 3.1-4 shows the crown shape and secondary splashing when a drop of milk impacts a thin liquid film. This image was first printed on an American postcard, which shows the interest in studying the drop impact characteristics (Yarin, 2006).

The single drop cases presented in this thesis have been derived from the Hillen, 2013 thesis. When a drop impacts the heated liquid film, a cavity is formed which consists of some of the heated film liquid, as well as some drop liquid in the thin liquid film beneath the cavity. Due to the contact of heated liquid film and cool drop liquid, an increased transient local heat flux is developed. Sarkar and Selvam (2009) found in their simulations that the sub-cavity region has a higher local heat flux compared to the surrounding liquid. This is due to the higher temperature difference between cold drop liquid and the heated film liquid in the thin sub-cavity region. The liquid present in this cavity 
region is dried out more quickly due to onset of boiling than in the surrounding liquid, thereby contributing to onset of CHF.

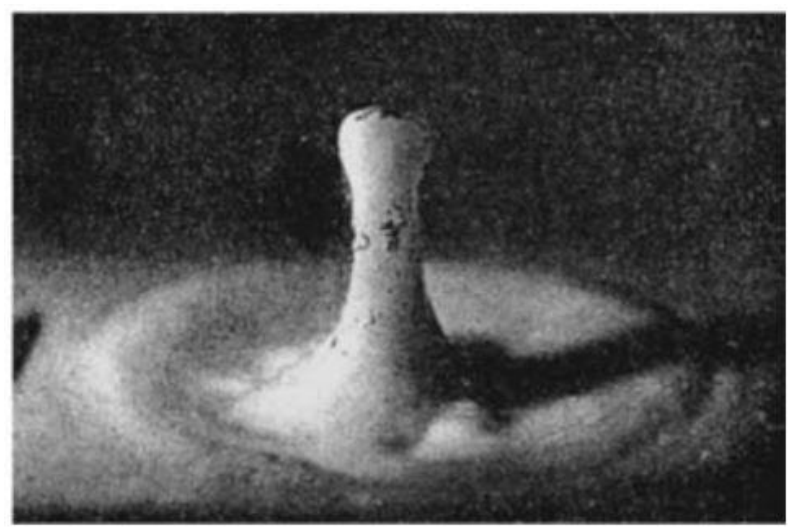

Figure 3.1-3: Worthington jet formed when a water drop impact on a deep pool of milk mixed with water (Yarin, 2006).

The forces of surface tension and gravity pulls the liquid in the crown downwards to refill the cavity, and thus the cold liquid again comes in contact with the heated surface, increasing the heat flux. This became the motivation for the present work: because the local heat flux is increased in the sub-cavity region, it is necessary to quantitatively compute the amount of liquid present in the sub-cavity region. Gehring et al., (2010), also used OpenFOAM by coupling the energy equation with the VOF model, to qualitatively investigate the region of interest while studying spray cooling.

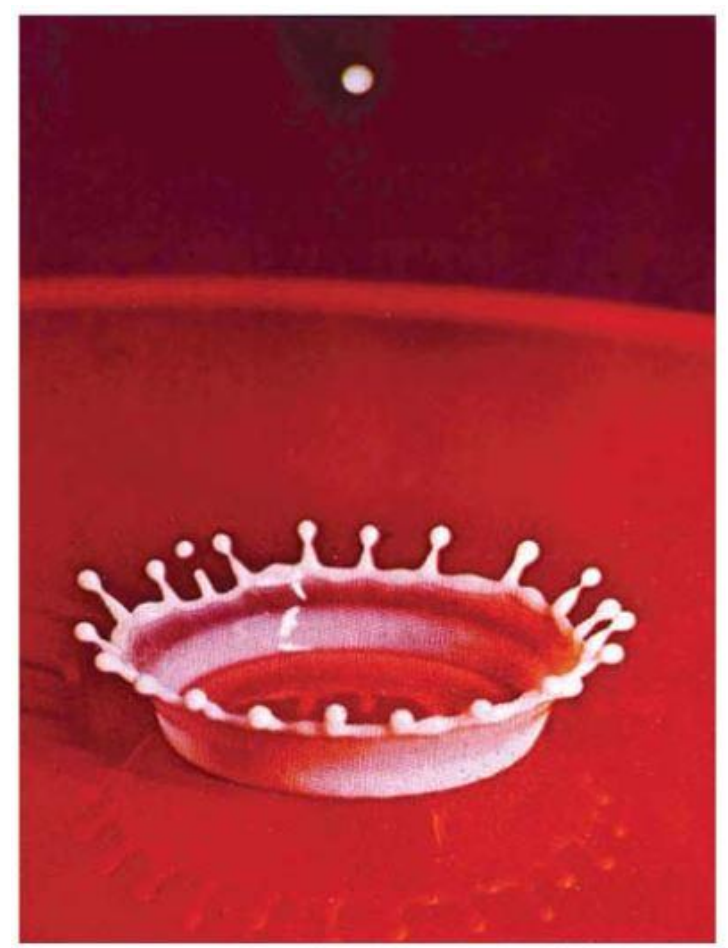

Figure 3.1-4: Drop impact pictured on an American postcard. Courtesy of Harold \& Ester Edgerton Foundation (2005) and Palm Press, Inc. (Yarin, 2006). 


\section{Volume of Fluid (VOF) Model}

The VOF model (Hirt et al., 1981) is a widely used method in the field of Computational Fluid Dynamics (CFD), which involves tracking of the interface between liquid and gas or two immiscible liquids. Applications of this model are columns of air bubbles rising in the liquid, liquid sloshing in a tank, and the dam break problem. This model is based primarily on computing the liquid volume fraction that is contained in a cell. Checking for the presence of liquid in a particular mesh cell is simple, but when the mesh is so refined and has many cells looking for an interface consumes a lot of computational power and time. Instead, a variable known as the liquid volume fraction is created to identify the interface between the gas and liquid in a cell. When the cell is filled with the liquid, this variable takes the value of 1 and when the cell is without any liquid, it takes the value of 0 . If the variable has a value between 0 and 1, then the gas-liquid interface must exist somewhere in that cell.

In this work for validation of experimental cases, 2D axisymmetric cases and 3D simulations were simulated using the ANSYS Fluent and OpenFOAM CFD codes, respectively. The following sections discuss the equations involved in the VOF model in both of the codes.

\subsection{VOF model in ANSYS Fluent}

Taking a set of momentum equations and a variable for monitoring the volume fraction, this model can model behavior of two or more immiscible fluids for the entire domain. The number of variables used for volume fraction increases with increase in the number of different fluids that are considered. On the whole, the sum of all the volume fractions must sum up to unity in every cell. For a volume fraction for fluid " $n$ ", the volume fraction, $\propto_{n}$ satisfies:

$$
\begin{aligned}
& \qquad \begin{array}{l}
\alpha_{n}=0 \rightarrow \text { the cell is empty of } n^{\text {th }} \text { fluid } \\
\propto_{n}=1 \rightarrow \text { the cell is full of } n^{\text {th }} \text { fluid }
\end{array} \\
& 0<\propto_{n}<1 \rightarrow \text { the cell has a liquid interface of } n^{\text {th }} \text { fluid with other fluid(s) }
\end{aligned}
$$

The location of the interface between phases is determined by solving the continuity equation for each phase. The equation for the $n^{t h}$ phase is, 


$$
\frac{1}{\rho_{n}}\left[\frac{\partial\left(\propto_{n} \rho_{n}\right)}{\partial t}+\nabla \cdot\left(\propto_{n} \rho_{n} \vec{v}_{n}\right)=S_{\alpha_{n}}+\sum_{m=1}^{r}\left(\dot{m}_{m n}-\dot{m}_{n m}\right)\right]
$$

where $\dot{m}_{m n}, \dot{m}_{n m}$ are mass transfer rates from phase $\mathrm{m}$ to $\mathrm{n}$ and phase $\mathrm{n}$ to $\mathrm{m}$, respectively. These are set to zero since there is no mass transfer in the present simulations. Also, $S_{\alpha_{n}}$ is the source term, which is also set to zero since there is no production of $n^{\text {th }}$ phase, and $\rho_{n}$ is the density of the $n^{\text {th }}$ phase.

The sum of all volume fractions must be unity in every cell throughout the domain.

$$
\sum_{n=1}^{r} \alpha_{n}=1
$$

Due to (3-3), there is one less continuity equation to be solved than the number of fluids. The above continuity equation can be solved either implicitly or explicitly. There are many discretization schemes available to discretize the equation in an Implicit scheme as well as in an Explicit scheme. There is a very different way of treating the interface between the 2 phases. A reconstruction scheme known as the Geometric Reconstruction scheme (Youngs, 1982) is used. The goal for this scheme is to calculate the interface shape and location precisely. The implicit and explicit schemes do not treat the cells containing an interface differently from the cells filled with liquids completely or empty cells. A piecewise-linear approach is used to represent the interface. A linear slope is assumed in each cell for the interface of the phases. This linear shape is then used for the calculation of mass transfer from the cell faces.

Scalar properties in the transport equations are calculated using the respective volume fractions for the phases present in the cell. The following equations give the volume-fraction averaged quantities for the cells throughout the domain.

$$
\begin{gathered}
\rho=\sum_{n=1}^{r} \alpha_{n} \rho_{n} \\
T=\sum_{n=1}^{r} \alpha_{n} T_{n}
\end{gathered}
$$




$$
\mu=\sum_{n=1}^{r} \alpha_{n} \mu_{n}
$$

For the velocity field, a momentum equation is solved throughout the domain, where this momentum equation is coupled to the volume fraction through density and dynamic viscosity terms.

$$
\frac{\partial(\rho \vec{v})}{\partial t}+\nabla \cdot(\rho \vec{v} \vec{v})=-\nabla p+\nabla \cdot\left[\mu\left(\nabla \vec{v}+\nabla \vec{v}^{T}\right)\right]+\rho \vec{g}+\vec{F}
$$

where $\vec{F}$ is the body force.

When dealing with fluids, the other two important factors which determine the dynamics of flow are values of the surface tension and the contact angle. Surface tension only acts at the interface between the phases. There are two models used in ANSYS Fluent to model surface tension: the Continuum Surface Force (CSF) model and the Continuum Surface Stress (CSS) model (Brackbill, 1992). The CSF method uses the non-conservative form for surface tension, which is very useful for constant surface tension problems. When a variable surface tension case is of interest, the method requires an additional surface tension component to be calculated in the tangential direction. The CSS method uses the conservative form for surface tension. Using this method, one need not calculate the curvature of the interface, which reduces the time required for computation. No additional term must be calculated when using this method. Due to these advantages, the CSS method has been used in the simulations presented in this paper.

Contact angle is present between the gas-liquid interface and the wall with which it makes contact. The wall adhesion model in ANSYS Fluent is taken from Brackbill, 1992. Contact angle is used to determine the cell normals near the wall which in turn determines the curvature of the liquid near the wall, according to:

$$
\hat{n}=\hat{n}_{w} \cos \theta_{w}+\hat{t}_{w} \sin \theta_{w}
$$

where,

$\theta_{w}$ is the contact angle,

$\hat{n}_{w}$ is the unit vector normal to the wall,

$\hat{t}_{w}$ is the unit vector tangential to the wall, and 
$\hat{n}$ is the interface normal in the cell near the wall.

\subsection{VOF Model in OpenFOAM}

Similar to ANSYS Fluent, in OpenFOAM, a single momentum equation and $(n-1)$ continuity equations are solved throughout the domain. When two or more fluids are in a cell, then the physical properties are again calculated as weighted averages depending on the volume fractions of the fluids present in that cell. The momentum equation is given by (3-7).

$$
\frac{\partial \rho \vec{U}}{\partial t}+\nabla \cdot(\rho \vec{U} \vec{U})=-\nabla p+\nabla \cdot \mu \nabla \vec{U}+\rho \vec{g}-\overrightarrow{F_{S}}
$$

where $\overrightarrow{F_{S}}$ is the surface tension force and $\nabla \cdot \mu \nabla \vec{U}$ is the viscous force. The continuity equation is given by (3-8).

$$
\nabla \cdot \vec{U}=0
$$

Surface tension forces and viscous forces affect the equation through the volume fraction term. Surface tension force acts only at the interface between two phases. It is modelled similar to the Continuum Surface Force (CSF) model in ANSYS Fluent. Surface tension is calculated as:

$$
\overrightarrow{F_{S}}=\sigma \kappa(x) \vec{n}
$$

where,

$$
\begin{aligned}
& \vec{n}=\frac{\Delta \alpha}{|\Delta \alpha|} \text { is the unit vector normal to the surface, } \\
& \kappa(x)=\nabla \cdot \vec{n} \text { is the curvature of the interface. }
\end{aligned}
$$

OpenFOAM uses the same concept of deciding the presence of an interface in a cell as ANSYS Fluent implements. That is,

$$
\begin{aligned}
& \qquad \propto_{n}=0 \rightarrow \text { the cell is empty of } n^{\text {th }} \text { fluid } \\
& \propto_{n}=1 \rightarrow \text { the cell is full of } n^{\text {th }} \text { fluid } \\
& 0<\propto_{n}<1 \rightarrow \text { the cell has a liquid interface of } n^{\text {th }} \text { fluid with other fluids }
\end{aligned}
$$

The amount of fluid ${ }_{\mathrm{n}}$ present in a cell is determined by the following formula:

$$
F_{v o l_{n}}=\alpha_{n} V_{c e l l}
$$

where $V_{\text {cell }}$ is the volume of the cell. 
Using the scalar transport equation, the volume fraction can be solved using the continuity equation:

$$
\frac{\partial \alpha_{n}}{\partial t}+\nabla \cdot\left(\alpha_{n} \vec{U}\right)=0
$$

As ANSYS Fluent implements the Geo-Reconstruction Scheme for extra interface compression and accuracy, OpenFOAM also uses an extra term which is added to the above transport equation (3-12). This term is modelled such that it acts only at the interface.

$$
\frac{\partial \alpha}{\partial t}+\nabla \cdot(\alpha \vec{U})+\nabla \cdot\left(\alpha(1-\alpha) \overrightarrow{U_{r}}\right)=0
$$

where $\overrightarrow{U_{r}}$ is the velocity field needed to compress the interface.

Contact angle is another deciding factor which arises at the cells containing an interface near a solid wall. As described in Patrik (2010), the equilibrium contact angle is calculated as:

$$
\theta_{c}=\arccos \left(\frac{r_{A} \cos \left(\theta_{A}\right)+r_{R} \cos \left(\theta_{R}\right)}{r_{A}+r_{R}}\right)
$$

where $\theta_{A}$ is the advancing contact angle and $\theta_{R}$ is the receding contact angle. The two radii of curvature are calculated from:

$$
r_{A}=\sqrt[3]{\frac{\sin ^{3}\left(\theta_{A}\right)}{2-3 \cos \left(\theta_{A}\right)+\cos \left(\theta_{A}\right)}} \text { and } r_{R}=\sqrt[3]{\frac{\sin ^{3}\left(\theta_{R}\right)}{2-3 \cos \left(\theta_{R}\right)+\cos \left(\theta_{R}\right)}}
$$

The advancing contact angle, $r_{A}$, refers to the contact angle formed when the liquid is advancing, and the receding contact angle refers to the contact angle formed when the liquid is receding. 


\section{Problem Description}

This chapter introduces the problem that this thesis has focused on. Simulating sprays is time consuming and needs high computational power which is expensive. To reduce the cost and increase the efficiency, Monte-Carlo models have been developed by Kreitzer (2010) and by Hussain et al. (2014) to simulate sprays. Before studying the full complexity of sprays, the behavior of single drops impacting into a residual liquid layer has been simulated in the present work, to analyze the cavity lifetime, and the sub-cavity film thickness and sub-cavity liquid volume as functions of time. These results will be useful in the Monte-Carlo spray models.

Depending on the values of the droplet Reynolds number, Weber number, Froude number, and the non-dimensional height $(h / d)$ of the liquid film, there are 5 types of single drop cases that were measured in experiments and analyzed by Hillen (2013) for cavity lifetime, centerline film thickness, and sub-cavity liquid volume. Reynolds number in this study varied from 1189 to 3555 , Weber number varied from 145 to 984 , and Froude number based on drop diameter varied from 94 to 510 . The initial two numbers are dominant in determining the cavity lifetime, centerline film thickness and sub-cavity liquid volume from case to case.

The five cases have been categorized in this study by Weber number, starting with low Weber number as case 1 to high Weber number as case 5 . Within each case, there are again 3 sub-cases depending on non-dimensional liquid layer height taking the values of $h / d$ equal to 1.0 as case $\mathrm{A}, 0.5$ as case $\mathrm{B}$, and 0.2 as case C. Based on these values of non-dimensional numbers, dimensional numbers such as impact velocity, diameter of the drop, and film thickness are computed. Table 4-1 gives details of the cases that have been studied by Hillen (2013), and simulated in the present work for comparisons. From these single droplet studies it is hoped that we will be able to predict the behavior of the full spray, and then the amount of heat transferred from the heated surface. All of these cases have been experimented and simulated at isothermal conditions. 
For the purpose of initial validation of the codes used in this paper, a case from the experimental work of Hillen et al. (2012) at $W e=141, R e=5700$ and $F r=103$ has also been simulated, again assuming isothermal conditions and all the parameters being the same as in the experiments.

The main focus of this study is to obtain the sub-cavity liquid volume versus time, which is necessary in order to estimate local heat flux that is required to evaporate this sub-cavity volume of liquid from the heated surface. Through this process, the temperature of the heated surface is controlled.

Table 4-1: Dimensional and non-dimensional parameters of simulated single drop cases; from experiments by Hillen (2013).

\begin{tabular}{|c|c|c|c|c|c|c|c|c|c|}
\hline \multicolumn{2}{|c|}{ Case } & $h_{\mathbf{0}}{ }^{*}$ & $\boldsymbol{h}_{\mathbf{0}}(\boldsymbol{\mu m})$ & $\mathbf{d}(\mathbf{m m})$ & $\begin{array}{c}\text { Vel } \\
(\mathbf{m} / \mathbf{s})\end{array}$ & We & Re & Oh & Fr \\
\hline \multirow{2}{*}{1} & $\mathrm{~A}$ & 1.0 & 3,019 & 3.028 & 1.727 & 145 & 1189 & 0.0101 & 100 \\
\cline { 2 - 10 } & $\mathrm{C}$ & 0.2 & 723.5 & 3.026 & 1.667 & 135 & 1176 & 0.0099 & 94 \\
\hline 3 & $\mathrm{~B}$ & 0.5 & 1,549 & 3.084 & 3.546 & 621 & 2643 & 0.0094 & 415 \\
\hline \multirow{2}{*}{5} & $\mathrm{~A}$ & 0.9 & 3,160 & 3.519 & 4.196 & 993 & 3574 & 0.0088 & 510 \\
\cline { 2 - 10 } & $\mathrm{C}$ & 0.2 & 707.7 & 3.518 & 4.177 & 984 & 3555 & 0.0088 & 407 \\
\hline
\end{tabular}




\section{Implementation of the Codes}

\subsection{ANSYS Fluent}

The commercial ANSYS Fluent 14.5 code has been used for all 2D axisymmetric simulations in the present work. As discussed in the Chapter 3, a VOF model has been used for the cases that are simulated in the present study. The number of Eulerian phases specified are three; i.e., taking drop liquid and film liquid separately as two different phases, plus one phase as air. For the purpose of the interface tracking scheme, an Euler explicit scheme has been used. This scheme has been briefly explained in Chapter 3. A value equal to $1 \mathrm{e}-6$ has been used as the lower cutoff limit for the volume fraction values. The lower cutoff limit defines any value of volume fraction in the entire domain that is less than this value to be zero, while the upper limit is taken as 1 . The maximum Courant number at the free surface is specified as 0.25 . Due to the presence of gravity and surface tension forces, there is a need for a body force formulation to be implemented in the model. Due to the poor convergence of algorithms, an Implicit Body Force model is implemented (ANSYS Fluent Guide, 2015), which makes the solution more robust by adding an extra term in the face flux equation (ANSYS Fluent Guide, 2015) for the correction of the body forces by correcting cell pressure and face flux. This correction will impose a realistic pressure field for the flow in the early iterative process.

The same material properties were given for both drop liquid and film liquid even though they were defined as two different phases; see Table 5-1.

Table 5-1: Properties of the liquid and air used in ANSYS Fluent.

\begin{tabular}{|c|c|c|}
\hline Property/Fluid & Liquid & Air \\
\hline Density, $\rho$ & $997 \mathrm{~kg} / \mathrm{m}^{3}$ & $1.2041 \mathrm{~kg} / \mathrm{m}^{3}$ \\
\hline Dynamic Viscosity, $\mu$ & $0.000998 \mathrm{~kg} / \mathrm{m}-\mathrm{s}$ & $1.8 e-05 \mathrm{~kg} / \mathrm{m}-\mathrm{s}$ \\
\hline reference temperature, $T$ & $288 \mathrm{~K}$ & $288 \mathrm{~K}$ \\
\hline Surface tension, $\sigma$ & \multicolumn{2}{|c|}{$0.0723 \mathrm{~N} / \mathrm{m}$} \\
\hline
\end{tabular}

For faster calculations, the CSS model (Brackbill, 1992) with wall adhesion has been used for surface tension force modelling. Wall adhesion has been included to add contact angle information. To increase the wetting of the surface, the contact angle between the bottom wall and the liquid (drop and film) has been set to $2^{\circ}$ with respect to air. With the default contact angle, liquid separation on the 
wall was observed. This would lead to a dry out of the cavity even without any heat from the bottom surface.

The overall domain dimensions are $16.5 d$ tall by $16.5 d$ radially. Boundary and initial conditions are shown in Figure 5.1-1. The right side of the domain is given an axis boundary condition. The bottom and left side of the domain are given a wall boundary condition. On these walls, wall adhesion is added to specify the contact angle of the liquid with respect to air. The top of the domain is given as a pressure outlet boundary condition. The domain has been extended enough for the cavity to collapse before the waves from the drop impact are reflected back to the impact region from the side wall.

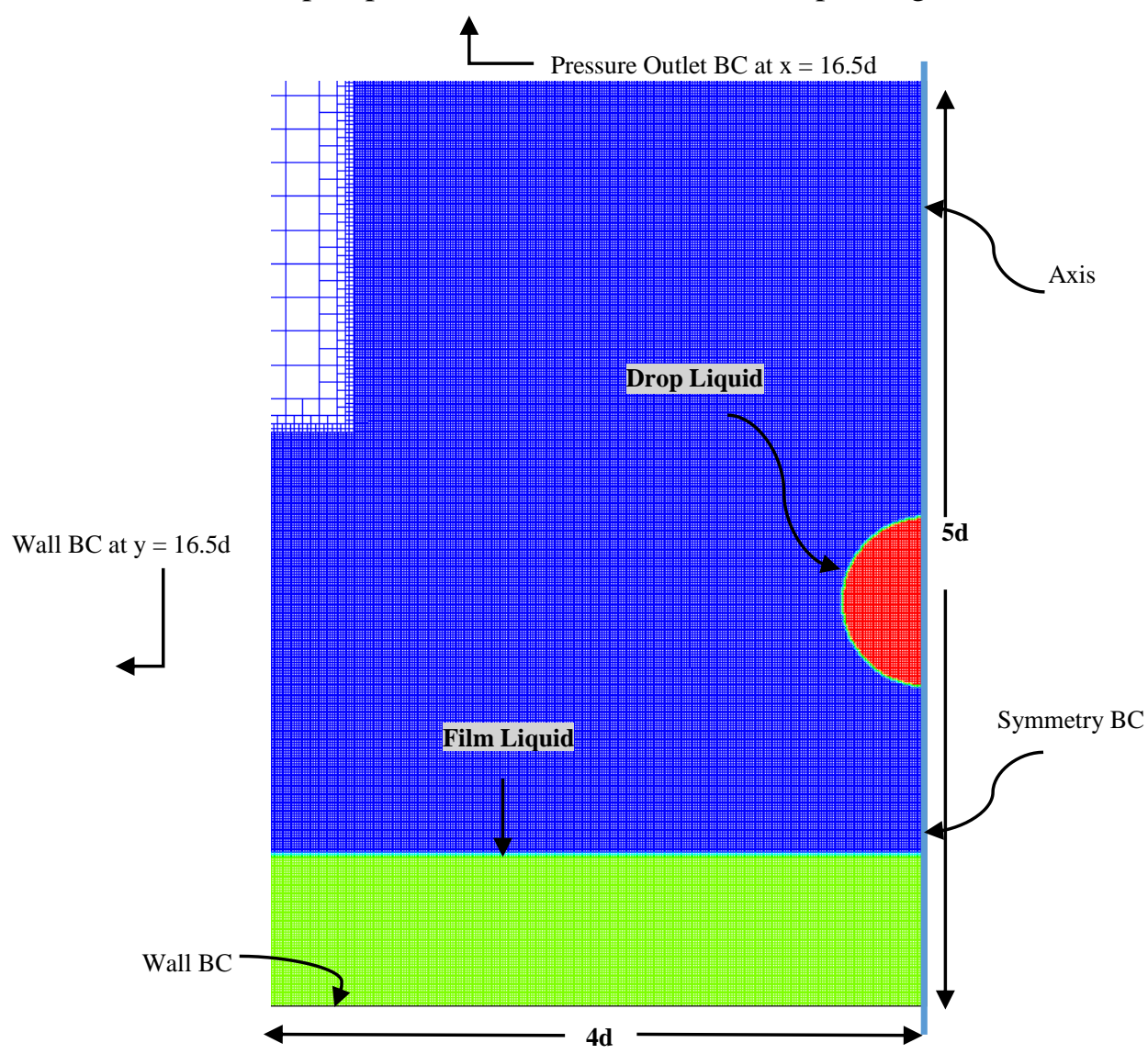

Figure 5.1-1: Boundary and initial conditions used in ANSYS Fluent 2D axisymmetric simulations.

A structured mesh of cell size $d / 5$ was created throughout the domain. This is the most coarse mesh. To capture the interface more precisely, a zonally refined mesh was created with a cell size of $d / 80$ up to a distance of $16^{*} d / 5$ from the bottom wall and from the right side of the domain. The transition of cell size from $d / 80$ to $d / 5$ is seen at the $17^{\text {th }} d / 5$ cell from the bottom wall. The change in the size of the mesh from $d / 80$ to $d / 5$ can be seen in the Figure 5.1-1. Due to the size of the domain compared to 
the smallest cell size, a cropped image of $5 d$ tall by $4 d$ radially is displayed. Red color indicates the drop liquid, green color indicates the film liquid and the blue color indicates air.

After solving the pressure-correction equation, the new velocities and the fluxes do not generally satisfy the momentum balance. Iterations need to be done to balance it. To avoid this, the Pressure Implicit with Splitting of Operators (PISO) algorithm (Issa, 1986) is used. The PISO algorithm is used for the pressure-velocity coupling in these simulations. Gradients are used for computing the scalar values at cell faces and also for secondary diffusion and velocity gradient terms. The Least Squares Cell-Based method (ANSYS Fluent Guide, 2015) is used to discretize these gradients. The PREssure STaggering Option (PRESTO!) scheme has been used for the discretization of the pressure equation, as implemented in the current version of Fluent. For the discretization of density and momentum, a second order upwind scheme is used.

As discussed in Chapter 3, ANSYS Fluent implements many different methods for the discretization of the volume fraction, but for the simulations presented in this thesis, the Geo-Reconstruction scheme (Youngs, 1982) is used. A first order implicit scheme is used for the transient formulation. Due to the limitations of the storage memory, the simulation data was saved at every 25 time steps, with a time step size of $5^{*} 10^{-6}$ seconds.

\subsection{OpenFOAM}

In an effort to remove the errors resulting from the non-physical constraint of axisymmetry when the crown becomes unstable and splashes, a full 3D simulation of each of the same cases has been performed using OpenFOAM, using the same Volume of Fluid model and two phases; i.e., liquid and air. As the simulation now became a 3D domain, there was the problem of the huge number of cells resulting in very long execution times. To run the job in ANSYS Fluent, it took a long amount of time to simulate even with a pie-shaped quarter domain. With the help of the High Performance Computing Center at West Virginia University we could run OpenFOAM with 8 processors. This simulation has been performed using the same parameters as for the 2D case (Figure 5.1-1). Figure 5.2-1 shows the domain setup used in OpenFOAM. All the side surfaces and the top surface of the domain are pressure outlet boundary conditions, while the bottom surface is a wall. The domain size 
is $36 * 18 * 36 \mathrm{~mm}$. Initial conditions were set to be the same as in the $2 \mathrm{D}$ axisymmetric case. The mesh used in OpenFOAM was set as a geometric progression in the vertical direction due to the limitations of computational power, and in order to obtain better resolution of the sub-cavity liquid film thickness. The lowest cell edge length was set equal to $d / 100$ at the bottom wall, increasing to $d / 5$ at the top wall.

To track the interface precisely between the liquid and the air, the interFoam solver is used. As explained in Chapter 3, the VOF model with interface compression scheme is used to track the interface. This scheme is modelled in such a way that it acts only at the interface, while outside the interface, there is no effect of it on the solution. Unlike ANSYS Fluent, there is no GUI for OpenFOAM and this is the reason why OpenFOAM is faster in computation. The RAM which is used for graphics in Fluent can't be used for computation. The overall structure of OpenFOAM is

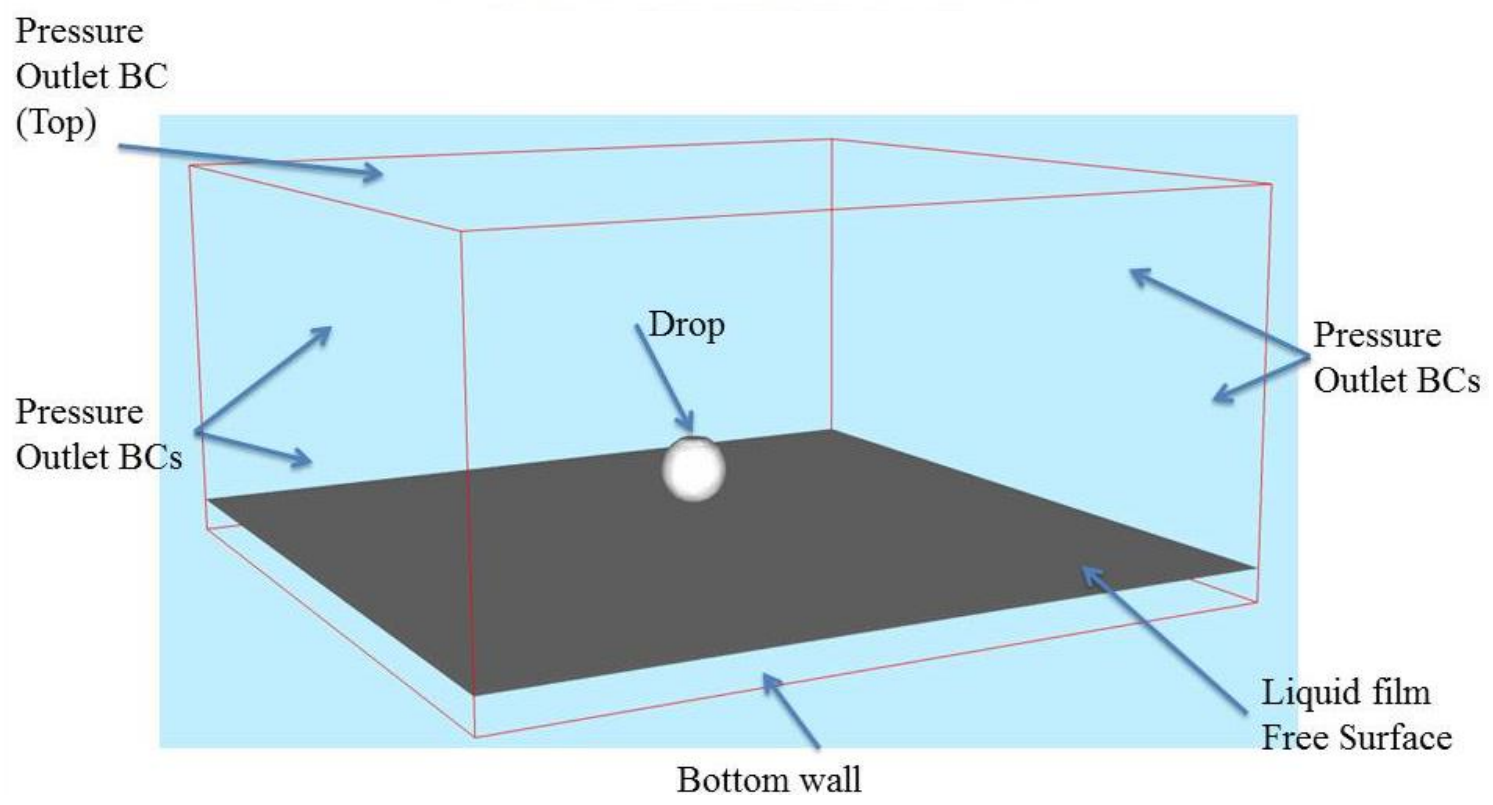

Figure 5.2-1: Boundary conditions and domain for 3D OpenFOAM simulations.

relatively simple. To setup a case, three folders are essential: 0 , constant, and system. The 0 folder contains the initial and the boundary conditions. It defines the flow state at the very beginning time of the simulation. The constant folder contains the information about the mesh, properties of fluids, simulation type, and gravity. The word constant is used for this folder because the values of all properties contained in this folder are constant throughout the simulation. The system folder contains 
the details of the type of solver, schemes, any post-processing codes, patching between the regions, duration of the simulation, and time step size.

\subsubsection{Domain and Mesh}

The file needed to specify the domain vertices, type of grading and which vertices connect to what face of the domain are specified in the file blockMeshDict which is present in the constant/polyMesh folder. The contents of the file are presented in Figure 5.2-2. The "convertToMeters" factor of 1 indicates that all vertices coordinates are specified in meters. The vertices of the domain are specified in the vertices section. The numbering of these vertices starts with 0 . There can be as many subdomains as needed in a simulation. In this thesis, the cases have been simulated with one domain so there is only one hexahedral cell that is created. Under the section blocks, a single domain with 8 vertices is created. The numbers in the first parenthesis represent the order of block creation and the second parenthesis gives the information of mesh points in $\mathrm{x}, \mathrm{y}$, and $\mathrm{z}$ coordinates, respectively. The simpleGrading term refers to how the mesh points are spaced in the respective axes. In $\mathrm{x}$ and $\mathrm{z}$ axes, the points are spaced evenly, while the points in the y axis are expanded as a geometric progression. The ratio of last cell edge length to the first cell edge length is the value to be specified. In the boundary section, the boundaries of the domain and the vertices which account for the respective faces are specified. Figure 5.2-3 shows the mesh created in OpenFOAM. The mesh near the bottom wall is so dense that the color of the film liquid near the bottom wall is faded away.

The type of mesh must be specified in OpenFOAM. Either the mesh is constant throughout the simulation ("static"), or the mesh changes versus time, which is the "dynamic mesh adaption" technique. This is specified in dynamicMeshDict file in the constant folder of the case directory, see Figure 5.2-4. All the simulations presented here have been performed in the static mesh environment. 


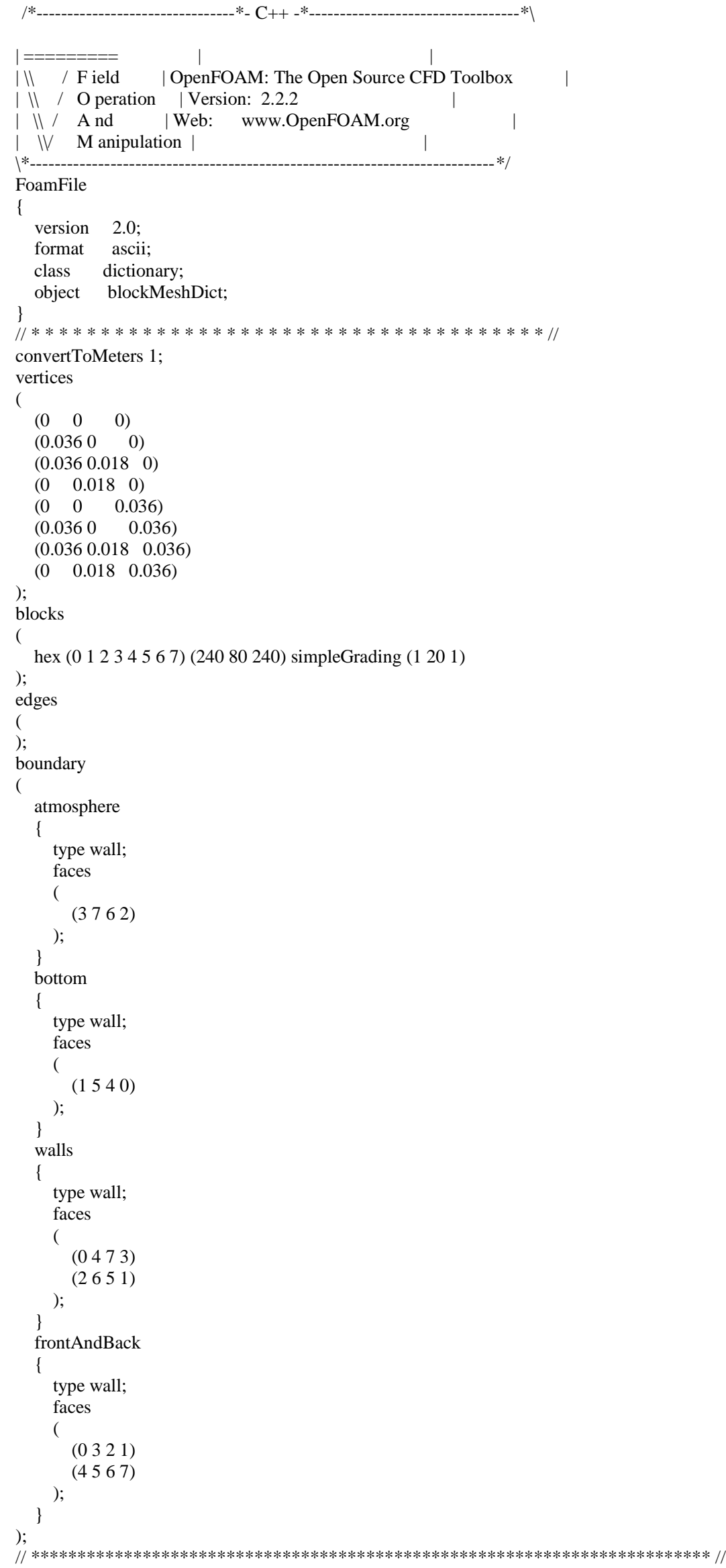

Figure 5.2-2: OpenFOAM file "blockMeshDict". 


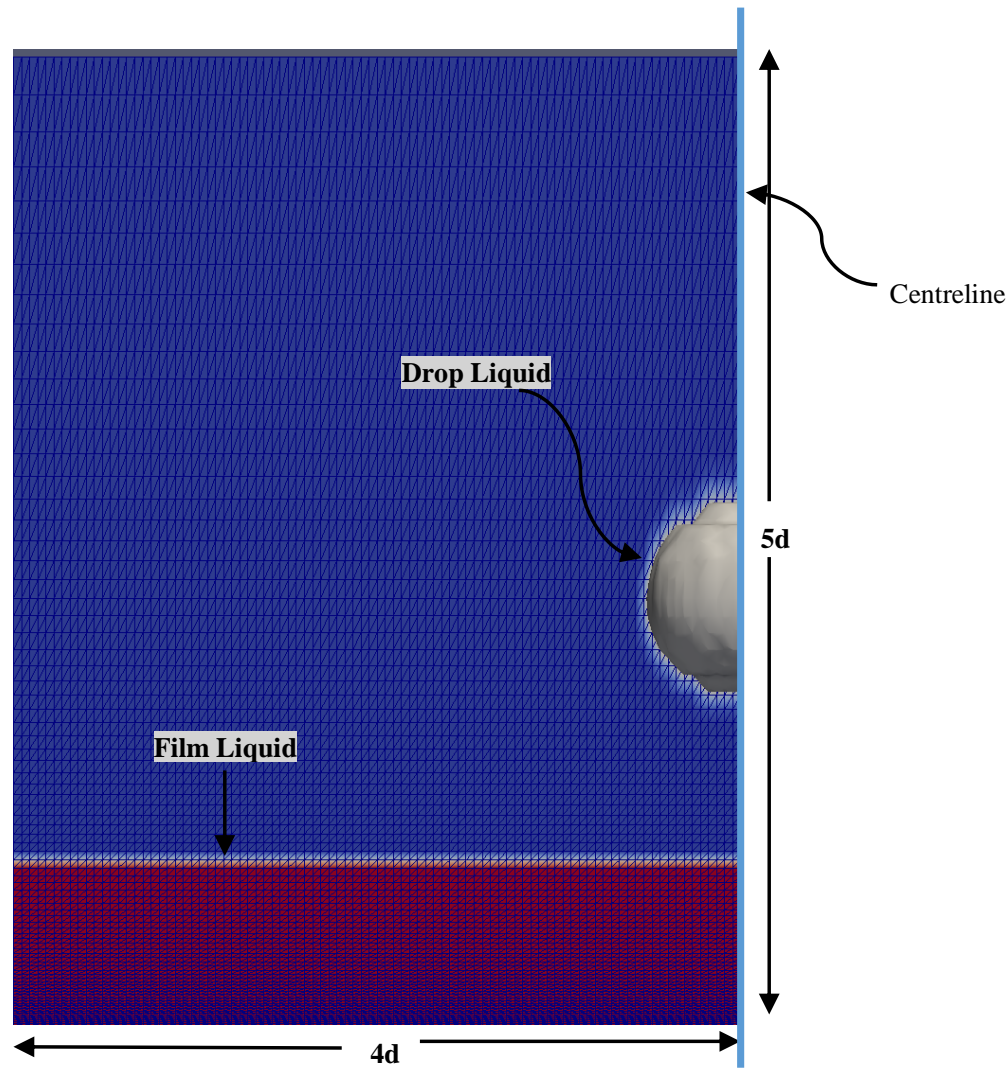

Figure 5.2-3: OpenFOAM mesh cropped and magnified to the same size as of Figure 5.1-1.

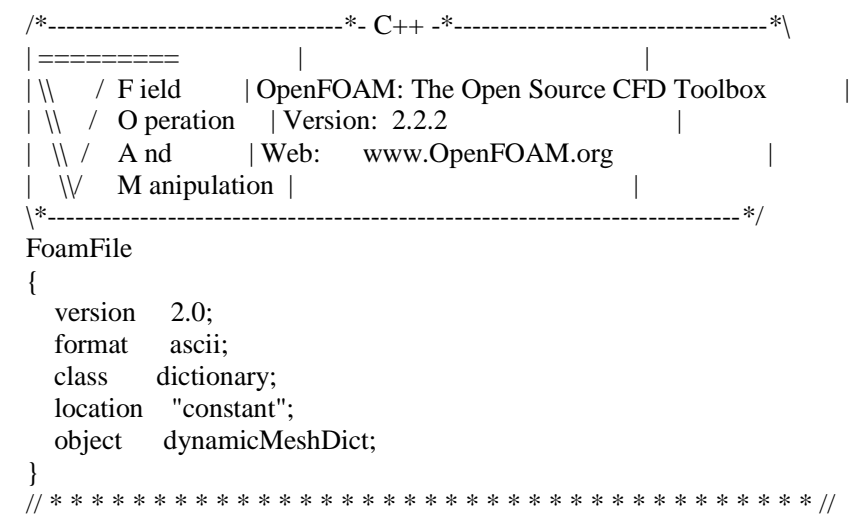

dynamicFvMesh staticFvMesh;

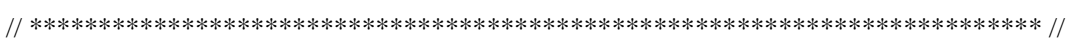

Figure 5.2-4: OpenFOAM file "dynamicMeshDict".

\subsubsection{Boundary Conditions}

After creating the faces, the type of boundary conditions have to be specified. These are done with file alphal present in 0 folder, see Figure 5.2-5. The following file shows the structure. For the bottom wall, a constant contact angle of $2^{\circ}$ has been specified. 


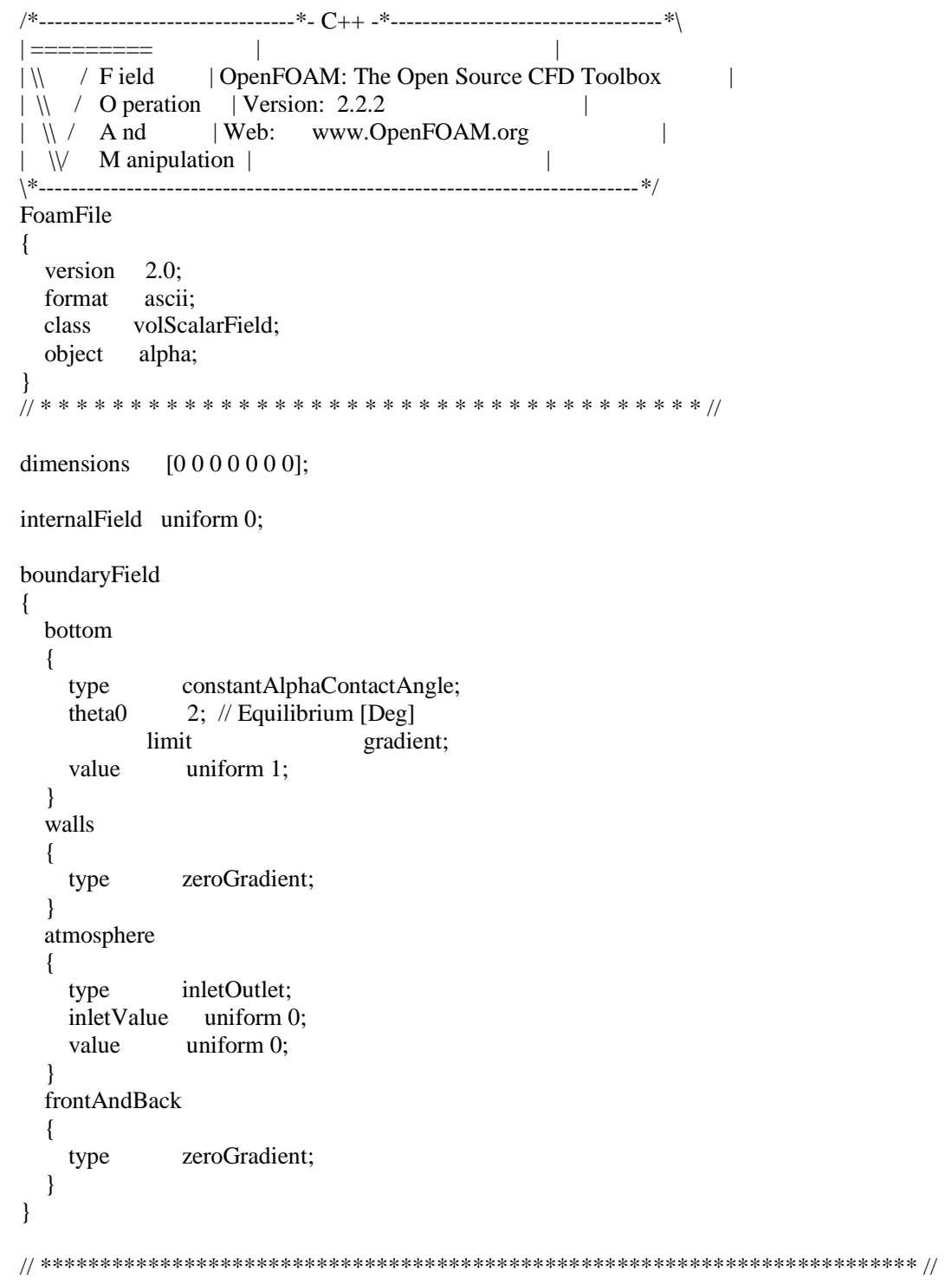

Figure 5.2-5: OpenFOAM file "alpha1".

\subsubsection{Transport Properties}

Even though all of the present cases have high Reynolds numbers, the simulations were run using a laminar model. The properties like surface tension, viscosity and density vary from case to case. These are specified in transportProperties, which is located in the constant folder, see Figure 5.2-6. Unlike in ANSYS Fluent, here we have to specify the kinematic viscosity (nu). 


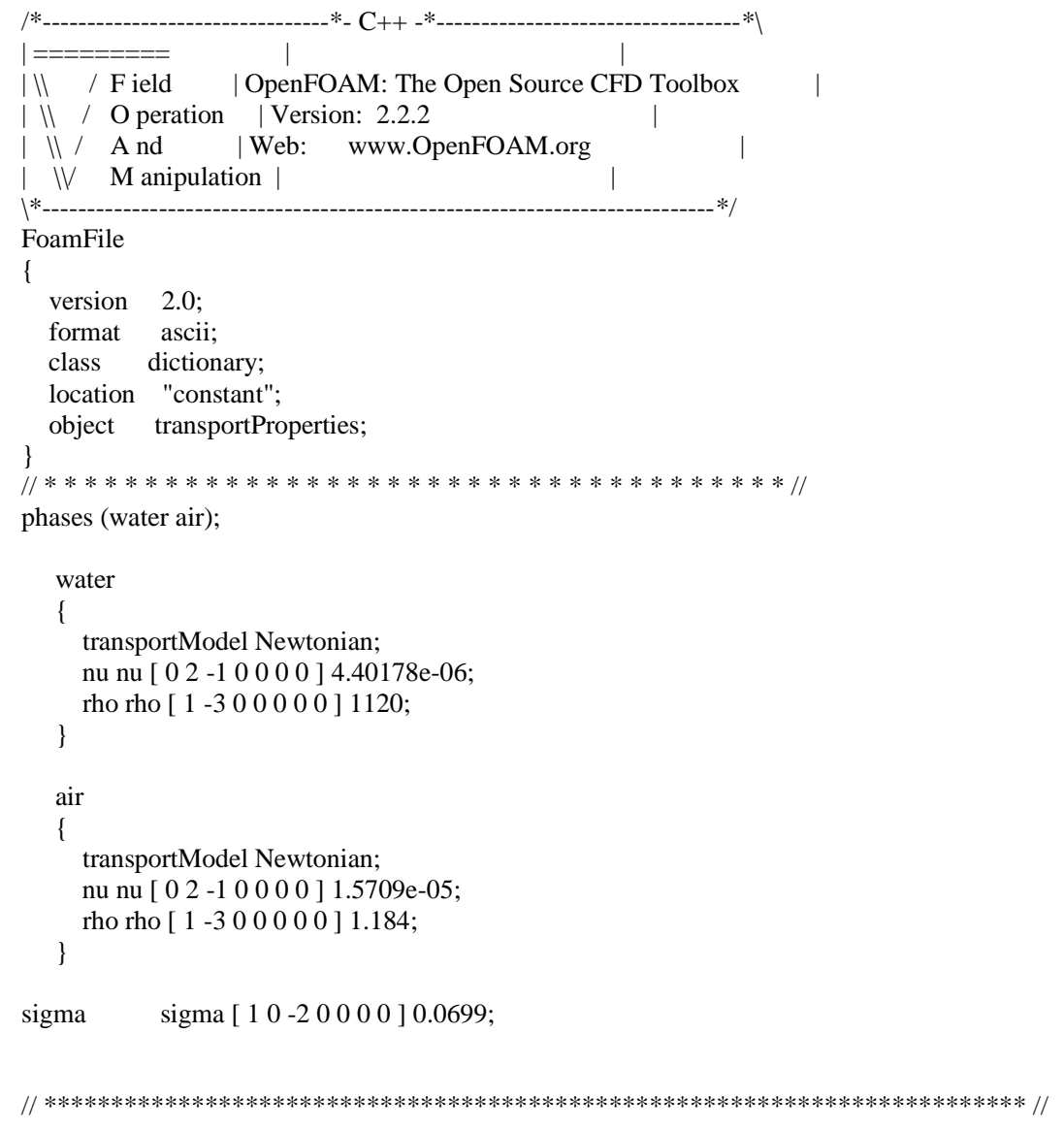

Figure 5.2-6: OpenFOAM file "transportProperties".

\subsubsection{Control Dictionary}

After specifying the conditions for the case, the next step is to specify the application, time step and data save intervals. These are written in controlDict, which is in system folder of the case folder, see Figure 5.2-7. The type of application used in the present simulations is interFoam. This is used for two incompressible, isothermal, immiscible fluids. A VOF phase-fraction based interface capturing approach is used in the simulations. When 'yes' is specified for runTimeModifiable, then it means that any changes to the controlDict file during the simulation will be implemented, while a 'no' to this condition implies that there will be no implementation in the current running simulation. The next condition adjustTimeStep indicates that the simulation time step can be modified during the simulation, keeping the constraint of maximum Courant by maxCo. The Courant number with the new time step will never exceed the number set by $\max C o$. The time step is also constrained by maxDeltaT, which explicitly dictates the maximum time step size. 


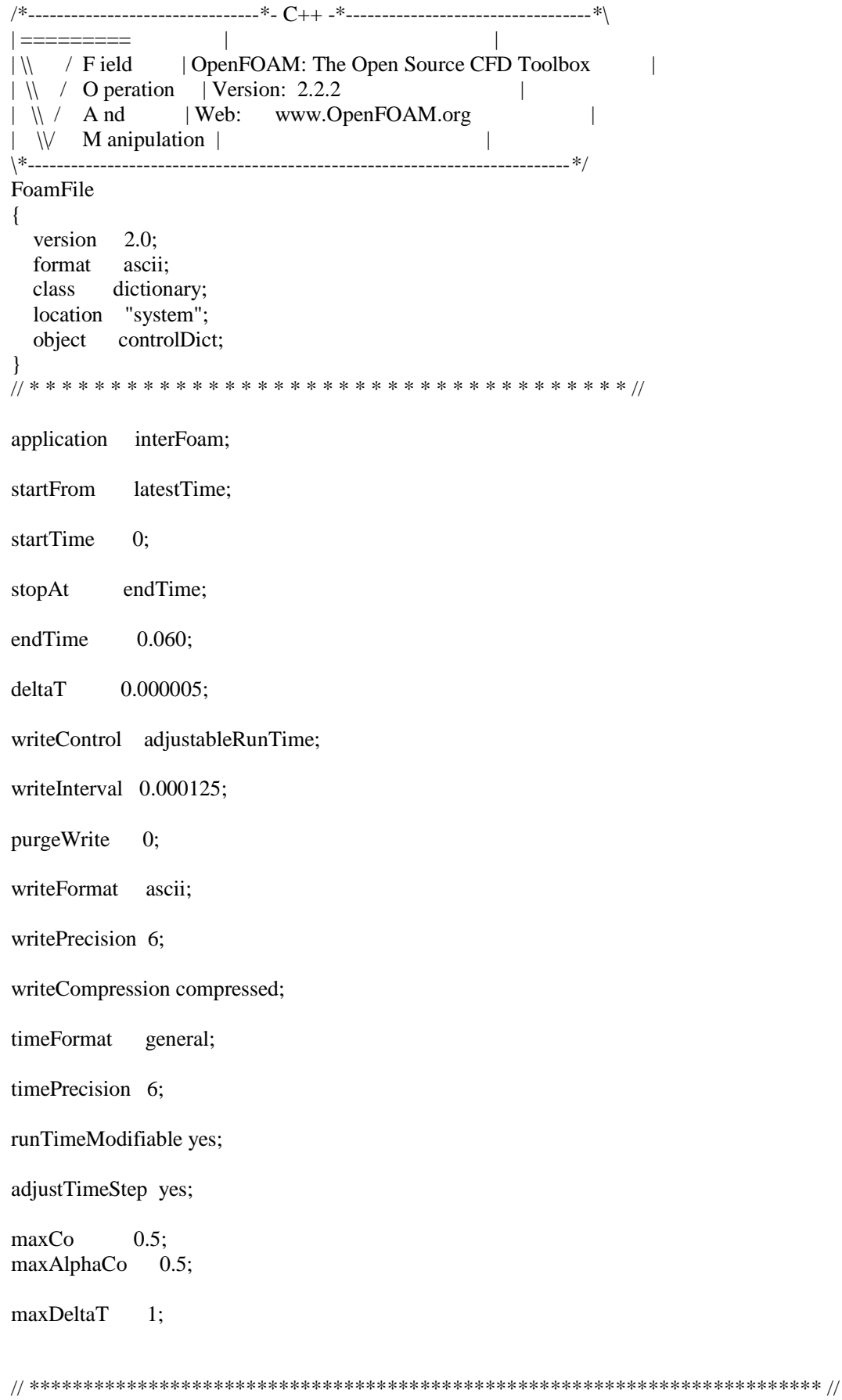

Figure 5.2-7: OpenFOAM file "controlDict".

\subsubsection{Parallel Computation}

The current mesh generated contains $4.6 \mathrm{M}$ cells which is a large number of cells to be simulated. At West Virginia University (WVU), a Super Computing System (Mountaineer) funded in part by the National Science Foundation EPSCoR Research Infrastructure Improvement Cooperative Agreement \#1003907, the state of West Virginia (WVEPSCoR via the Higher Education Policy Commission) and WVU was available to tackle this problem. Eight processors with a total of $48 \mathrm{~GB}$ of RAM has been 
used to compute the 3D solutions. The procedure to divide the domain into eight sub-domains is specified in the decomposeParDict file in the system directory, see Figure 5.2-8. A simple method is used in these simulations to divide the domain into sub-domains. The $n$ in simpleCoeffs, defines the number of divisions to be made in each axis direction. In the present simulations, a uniform discretization has been used along the axes. The term, delta, is the maximum distance between the divided sub-domains that can be applied.

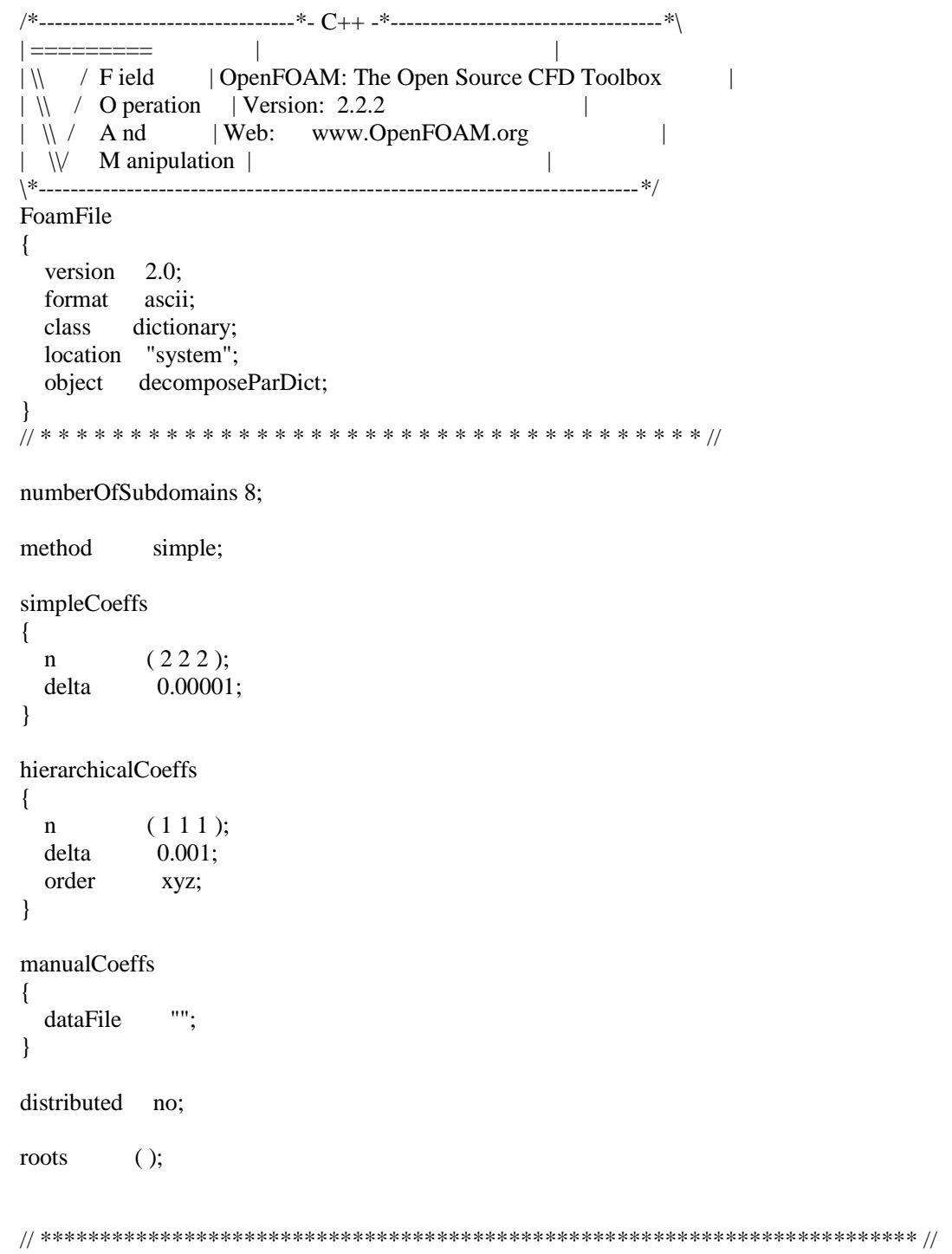

Figure 5.2-8: OpenFOAM file "decomposeParDict".

\subsubsection{Schemes}

A wide selection of numerical schemes are available for the user to select to numerically approximate the derivatives in the equations. These schemes are specified in the fvSchemes file present in the system directory, see Figure 5.2-9. Different schemes can be applied for different types of derivatives 
that are present in the equations. Standard Gauss finite volume integration is used in general. This scheme uses values on the cell faces and sums them up. These values are interpolated from neighboring cell centers. The interpolation is done as linear in the most of the cases.

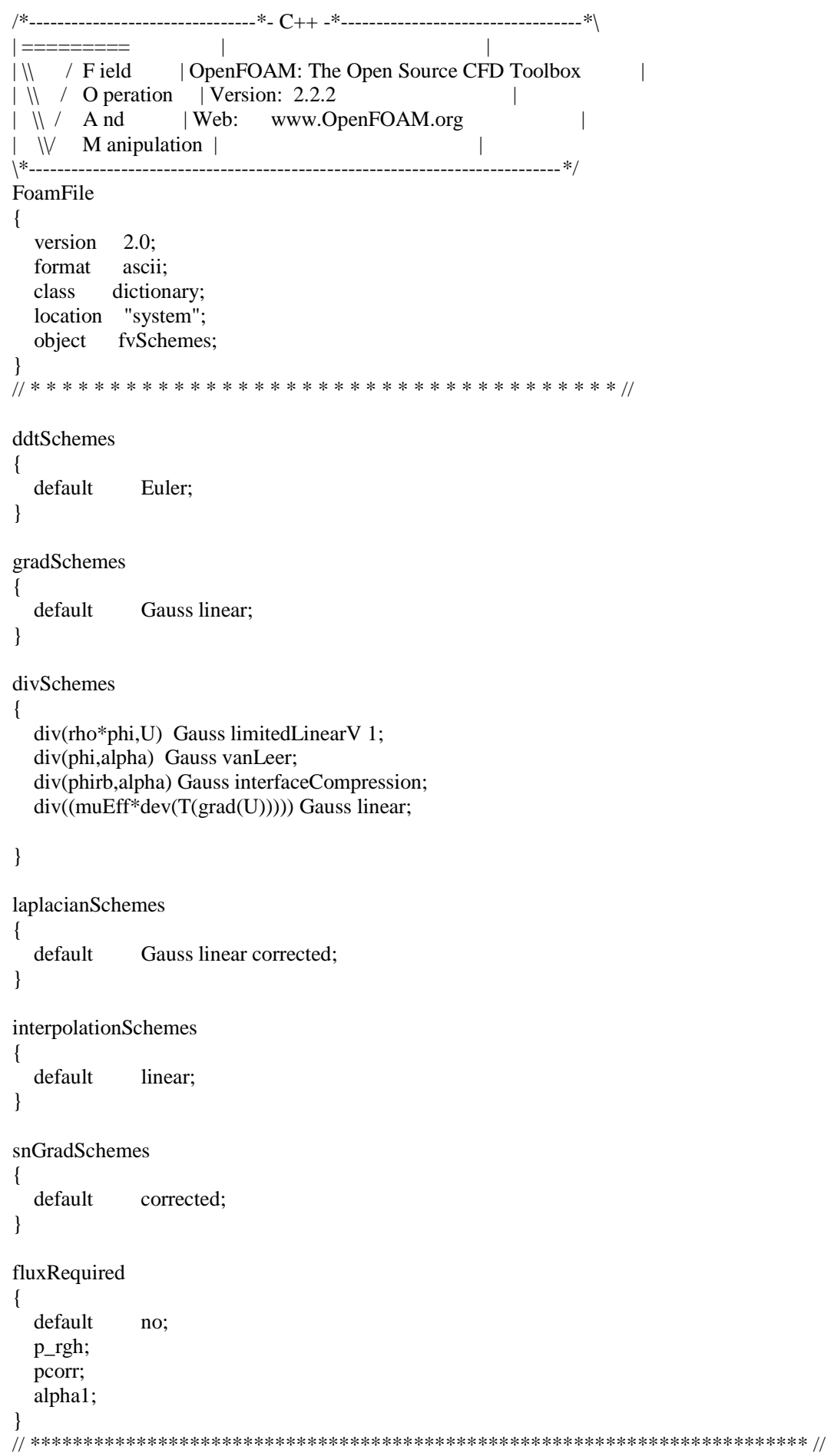

Figure 5.2-9: OpenFOAM file "fvSchemes".

For time derivatives an Euler scheme, which is an implicit and first order, is used. The gradient terms that are encountered by the OpenFOAM are approximated using a Gaussian scheme, and the 
interpolation between cell center and face center is carried out as linear by default. The convection terms are defined in divSchemes. The Gaussian scheme is used to approximate the divergence terms in the equations. An interpolation scheme is to be followed that gives the format as Gauss <interpolationscheme>. Gauss interfaceCompression is used to solve the alpha (volume fraction) equation. The convection term $(\nabla \cdot(\rho \vec{U} \vec{U}))$ in the momentum equation is represented as $\operatorname{div}(\operatorname{rho} * p h i, U)$. For the term $\operatorname{div}((\operatorname{muEff*} \operatorname{dev}(T(\operatorname{grad}(U)))))$ the numerical scheme used is Gauss linear.

Laplacian terms in the Navier-Stokes Equations are solved by the schemes defined in laplacianSchemes. The syntax to define the scheme is as follows: Gauss <interpolationScheme> $<$ snGradScheme >. For the interpolationScheme, linear scheme is used. snGradScheme represents the surface normal gradient terms, and it is evaluated at the cell face. For the snGradScheme, the corrected scheme is used, as it is unbounded, conservative and second order in behavior. By default interpolationSchemes are set to linear. And finally, the variables which require generation of flux are alpha, pcorr (pressure corrected) and p_rgh (pressure without hydrostatic pressure).

\subsubsection{Solvers}

The solvers needed to solve for the flow field variables, along with tolerances and algorithms are specified in fvSolution file which is located in the system directory; see Figure 5.2-10. In the beginning of every variable of the Figure 5.2-10, there is a keyword solver; this keyword specifies the method by which OpenFOAM has to solve the matrices that are obtained from the equations. Here interFoam solves the equations for $p c o r r, p \_r g h, p \_r g h F i n a l$ and $U$. Hence, there are entries for each variable specifying what type of solver, preconditioner and tolerance are to be used. For the variables pcorr, p_rgh and p_rghFinal, the Preconditioned Conjugate Gradient (PCG) (Caraba, 2008) method is used. This method was chosen for solving a very large sparse symmetric system because it is one of the most effective algorithms. For the variable $U$, the Preconditioned Bi-Conjugate Gradient (PBiCG) method is used. This algorithm is used to solve asymmetric sparse systems. A preconditioner is used to reduce the problem into a simple form that can be solved by numerical methods. 


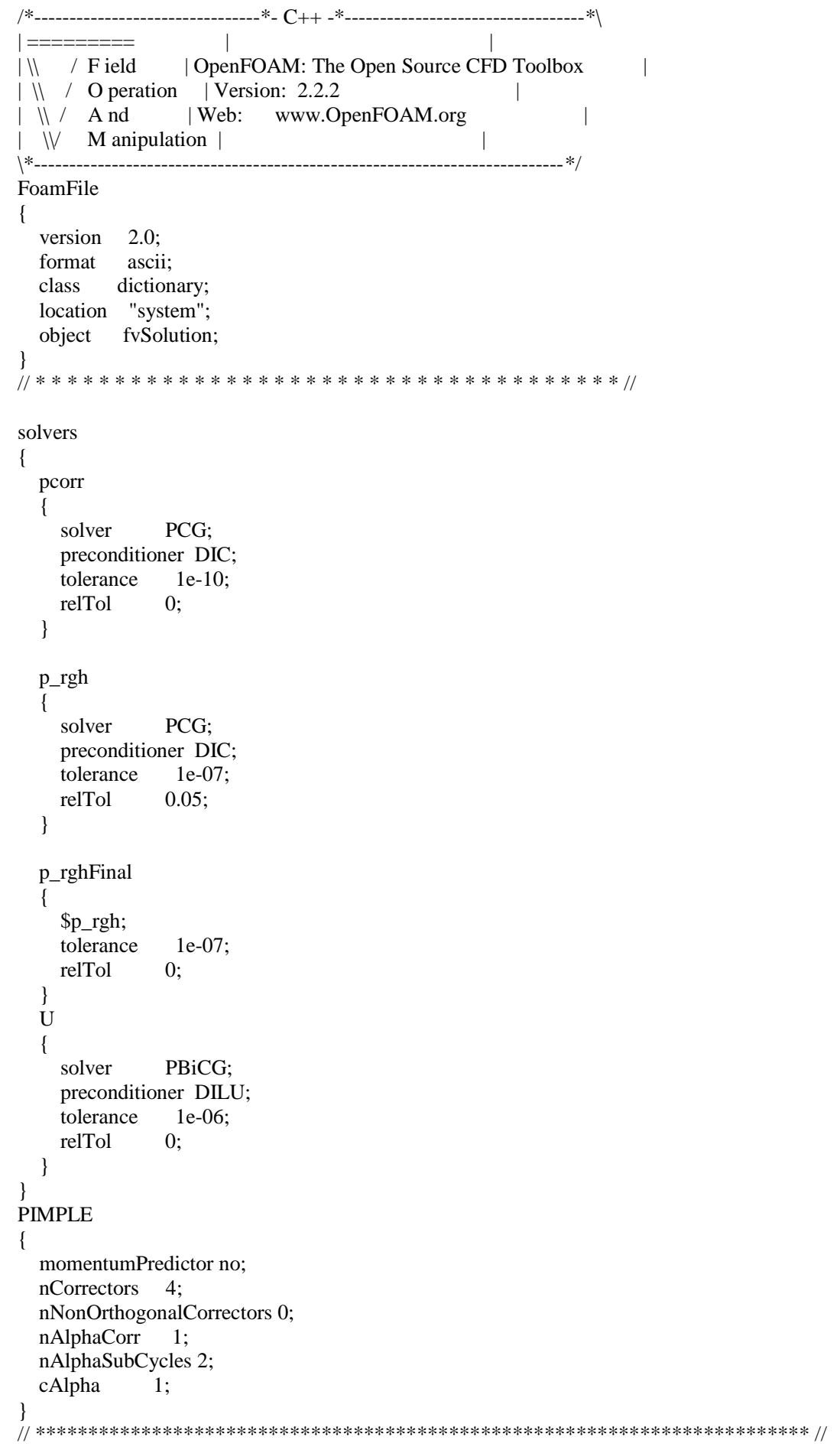

Figure 5.2-10: OpenFOAM file "fvSolution".

For pcorr, p_rgh and p_rghFinal, the Diagonal incomplete-Cholesky (DIC) preconditioner is used, due to its capability in preconditioning the symmetric matrices in conjugate gradient solvers. For $U$, the Diagonal incomplete- $L U(D I L U)$ preconditioner is used because of its capability to precondition asymmetric matrices. 
All the numerical methods used are iterative. When to stop the iteration is determined by the values of tolerance, relTol and maxIter. tolerance is similar to the residuals in ANSYS Fluent. Solution iteration is stopped when the residual value falls below the specified tolerance value, or when the ratio of current to initial residuals is less than the relTol, or when the number of iterations reaches the maxIter. The lower the tolerance or relTol is, the higher the solution accuracy is (keeping the computation time into consideration).

The PIMPLE algorithm has been used in these simulations, which is a combination of the PISO and SIMPLE algorithms. The PIMPLE loop is used for alpha (volume fraction) in the code. With the help of initial solutions, the PIMPLE algorithm evaluates and corrects the solution. The number of corrections to be made can be specified in the keyword nCorrectors. Since the cell faces of the mesh in these simulations are all aligned with the co-ordinate system (i.e. the face normal is parallel to the line joining the centers of the cells that the face joins) there is no need for any orthogonality correction. Hence zero is specified for the keyword nNonOrthogonalCorrectors. The alpha is to be calculated for the present time step to get accurate measurement of momentum transfer through the cell faces. To achieve this, within a time step, alpha is calculated many times depending on the number specified by the user for keyword nAlphaSubCycles. To improve the quality of alpha, it is iterated through fixed point iteration by the number specified for the keyword nAlphaCorr. The cAlpha keyword specifies the interface compression. 


\section{Post Processing Analysis}

\subsection{Centerline Film Thickness}

After completing the simulations, the next step is to analyze the simulation data to compute the desired cavity formation. For the purpose of getting the sub-cavity centerline film thickness data, the simulation data must be imported into different software depending on the simulation. In the case of the 2D axisymmetric simulations, i.e. the simulations that are performed in ANSYS Fluent, the simulation data is imported into ANSYS CFD-Post. An isosurface for the air volume fraction at a value of 0.5 is created. For $2 \mathrm{D}$ axisymmetric cases, the $\mathrm{x}$-axis becomes the centerline, and the intersection of $\mathrm{x}$-axis and isosurface gives the centerline film thickness. The process is done manually, where the simulation is first saved at an interval of $0.000125 \mathrm{sec}$. Due to the large amount of data, the centerline film thickness is recorded at every $0.002 \mathrm{sec}$ after the initial impact of drop.

In case of 3D simulations, i.e. the simulations done in OpenFOAM, the simulation data is imported into EnSight. The 3D simulation is also saved at an initial time interval of $0.000125 \mathrm{sec}$, and the centerline film thickness is then recorded at every $0.002 \mathrm{sec}$. The process of retrieving the thickness data for 3D simulations is a bit different compared to the procedure used for 2D axisymmetric cases. Here, first an isosurface is created for the air volume fraction at a value of 0.5 , then using the clip option the isosurface along with the whole domain is sliced exactly into two halves. The cutting plane is perpendicular to the static liquid film. A vertical reference line at the center of the clip is created; this line divides the clip in two halves. The intersection of the reference line and the isosurface gives the film thickness at the center of the drop impact.

\subsection{Sub-Cavity Volume}

The main goal of this work is to calculate the sub-cavity volume that is created after the drop impact onto the static liquid film, as a function of time. A critical problem in calculating the sub-cavity liquid volumes is defining in a robust and meaningful way the outermost radial location of the cavity, beneath which the volume is to be calculated. There generally is a film thickness fillet versus time at a fixed radius as the cavity propagates to the impact surface and transitions into a (nearly) uniform film thickness after droplet impact. From another point of view, there is also a fillet of liquid film thickness 
varying at an individual time of interest as the film transitions radially outwards into the crown wall. An example of this fillet can be seen in Figure $6.2-1$, for a simulation of droplet impact into a static liquid layer at $W e=141 ; R e=5700$; and $h / d=0.614$, at a time of $20 \mathrm{~ms}$ after the initial contact of the drop with the liquid layer, this case was studied experimentally and modelled via CFD by Hillen $e t$ at., (2012).

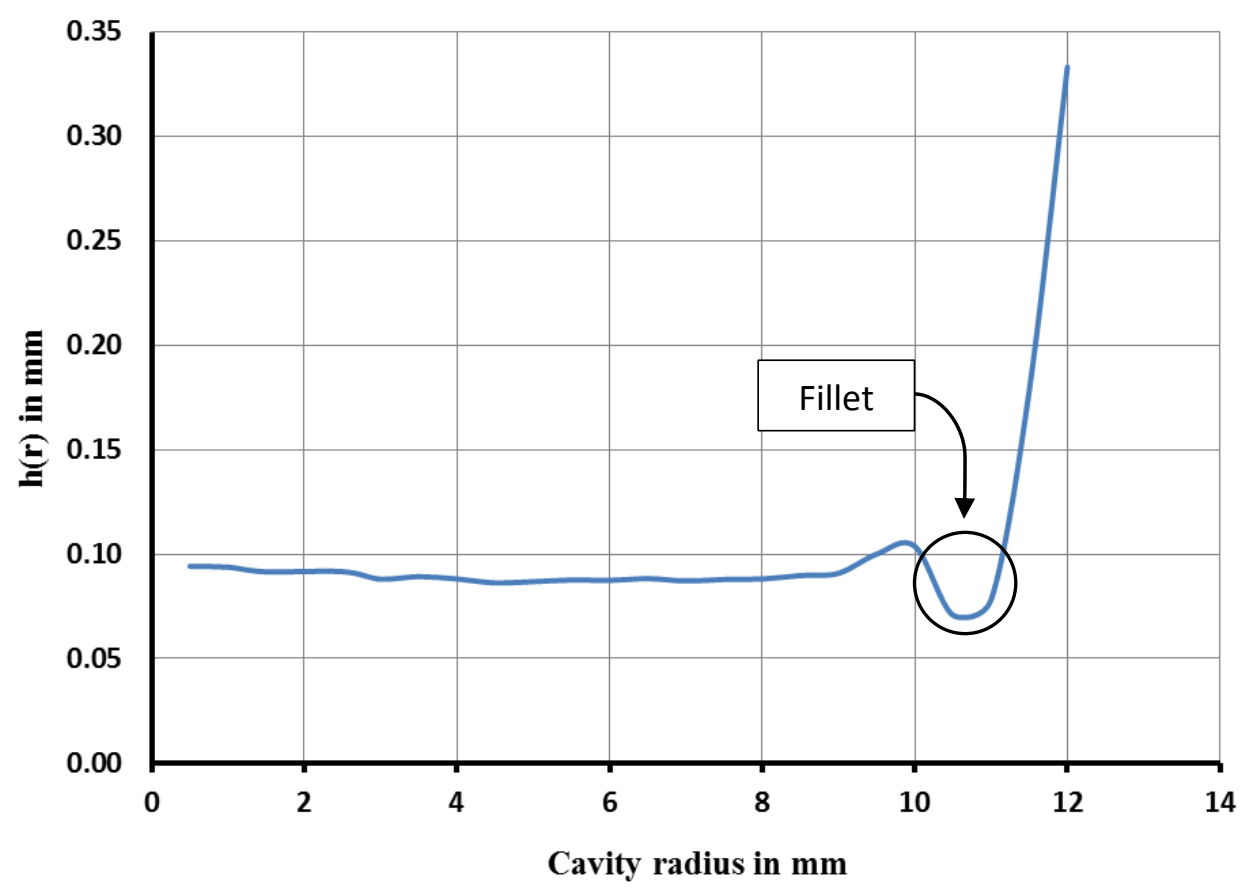

Figure 6.2-1: Example of the fillet at the outer radius of the liquid film thickness profile at $\mathrm{t}=20 \mathrm{~ms}$ where $\mathrm{h}(\mathrm{r})$ rises steeply; $\mathrm{We}=141, \mathrm{Re}=5700, \mathrm{~h} / \mathrm{d}=\mathbf{0 . 6 1 4}$.

Determining how much of the fillet where the film thickness begins to increase rapidly versus radius should be included in the volume calculation proved challenging, as most of the fillet is too thick and has too short of a lifetime compared to the sub-cavity film thickness to contribute to the cavity dryout. Rather than using an arbitrary set of conditions, or "judgment", to define the beginning and maximum liquid film thickness of the cavity, a radial location where the local film thickness first increases to be equal to a film thickness constraint value has been used. The film thickness constraint value, which is a cutoff height, is calculated using a running average of film thickness at every $0.002 \mathrm{sec}$ from the time of drop impact, multiplied by the constant values of 1.2, 1.3, 1.4, 1.6, 1.8 and 2.

The liquid film thickness profile in Figure 6.2-1 is exported from Ensight into an Excel file. The running average is calculated using a Matlab script by importing this Excel file. At each instant of 
time, the film thickness is averaged radially starting from the center of the cavity until the next radial location's film thickness is greater by a constraint value of 20,30,40,60, 80 and 100\% respectively, more than the current average. This method is compared to an alternate method used to calculate the cutoff height. In this method, the constraint limits under consideration were selected and averaged to get an average film thickness on the drop impact centerline and this average film thickness is multiplied by $1.2,1.3,1.4,1.6,1.8$ and 2 . In the first method, the cutoff height is different for every time step whereas in the second method, it remains constant for all the time steps in the simulation.

To calculate the sub-cavity volume, a 3D surface is needed. Using a Matlab script, the profile is rotated $360^{\circ}$ about the x-axis with 600 angular divisions. The resulting coordinates are exported to Surfer to create a 3D surface. The circle corresponding to the particular film thickness cutoff value of 1.3 times the running average of film thickness radially has been marked using the Surfer software package with a different color (yellow), as shown in Figure 6.2-2.

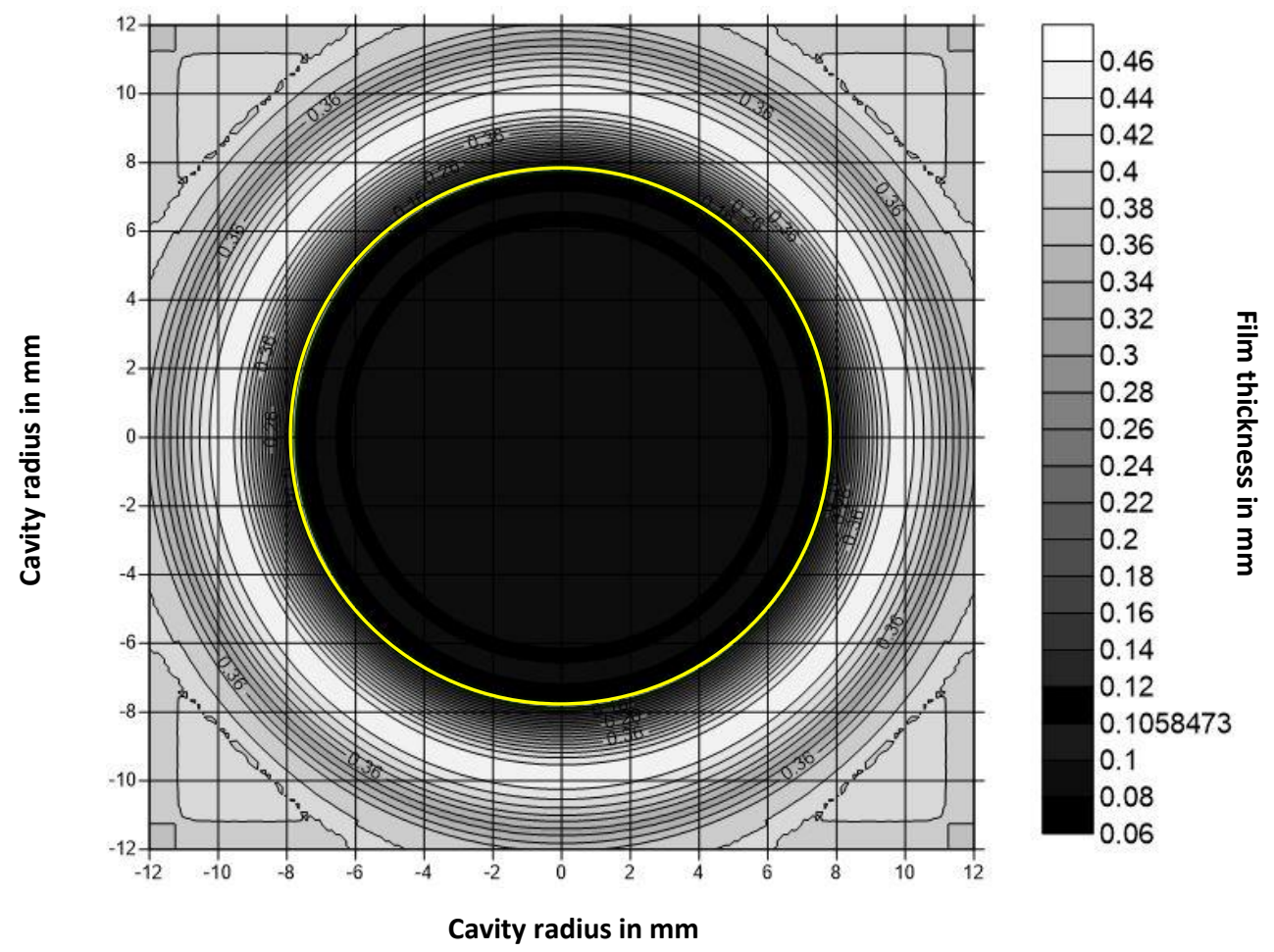

Figure 6.2-2: Radial location for outermost edge of sub-cavity region, highlighted in yellow, using cutoff value equal to 1.3 times the average centerline $\mathrm{h}$ at $\mathrm{t}=\mathbf{2 0} \mathrm{ms} ; \mathrm{We}=141, \operatorname{Re}=\mathbf{5 7 0 0}$, $\mathrm{h} / \mathrm{d}=\mathbf{0 . 6 1 4}$.

After creating the circle, and marking a set of points along this circle using the "digitize" feature in Surfer, to pick up the coordinates of the circle, then the outer region beyond the circle is "blanked", 
since the interest is only in calculating the volume of the sub-cavity region within the circle. This process is repeated for each of the other different multipliers defined above, and the respective volumes are calculated. The volumes are calculated in Surfer using the Trapezoidal Rule. This process of calculating sub-cavity volumes has been chosen because there is no liquid removed and no liquid is added, and accurate results are obtained. Initially, a method was attempted that avoided creating the circle, and filters were applied to remove all the liquid above the cutoff height, but this procedure resulted in unrealistic plots and results.

Figure 6.2-3 shows examples of the computed sub-cavity volume versus time using each of the different constraint multipliers of the running average of the film thickness in radial direction. The minimum film thickness constraint value (a multiplier equal to 1.0) proved inappropriate since this value failed to capture a significant portion of the sub-cavity volume of interest, while twice its value resulted in exaggerated results. The computed sub-cavity liquid volumes (Figure 6.2-3) match quite well for all constraint values, except for the times between approximately $15 \mathrm{~ms}$ and $35 \mathrm{~ms}$, where the largest variation is observed at around $25 \mathrm{~ms}$. This type of behavior can be explained by comparing the sub-cavity film thickness profiles versus radius at times in between this time interval. Two examples of these sub-cavity film thickness radial profiles can be seen in Figure 6.2-4 and Figure $6.2-5$, at times of $20 \mathrm{~ms}$ and $25 \mathrm{~ms}$, respectively, for a cutoff film thickness value of $1.6^{*} \mathrm{~h} \_\mathrm{min}$.

The horizontal axis in these plots has been set to $1.6 *$ h_min, which shows the intersection point with the profile below which the sub-cavity volume is calculated. Note that the profile at $20 \mathrm{~ms}$ is much steeper compared to the profile at $25 \mathrm{~ms}$. This difference in the slope of film thickness versus radius causes the increase in volume computed for times between $15 \mathrm{~ms}$ and $35 \mathrm{~ms}$, since increasing the cutoff film thickness value by a fixed percentage will increase the computed sub-cavity liquid volume more when the surface slope is smaller. From these observations, it was concluded that 1.3 times the running average liquid film thickness in the radial direction is an appropriate cutoff value to use for computing the sub-cavity liquid volumes for all future work, because of the relatively smooth time variation in the resulting liquid volumes. 


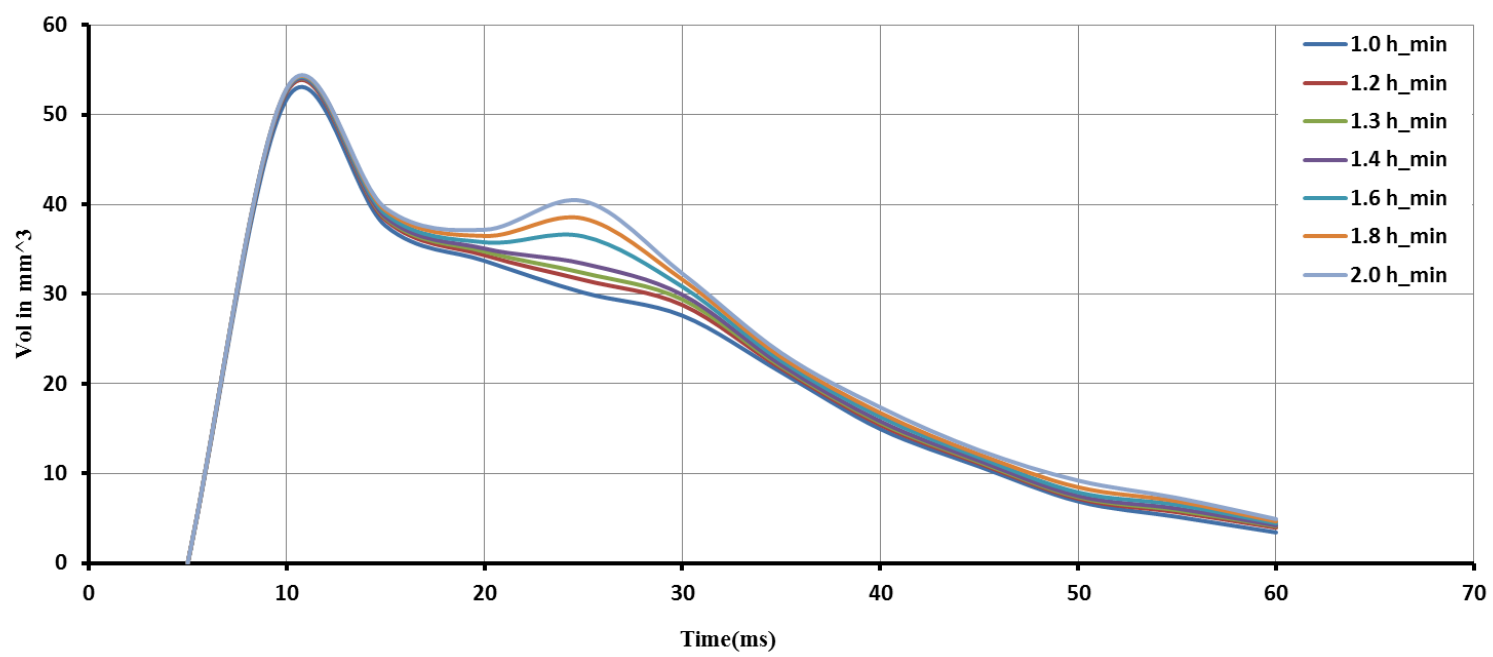

Figure 6.2-3: Computed sub-cavity liquid volumes vs. time, for a range of liquid fillm thickness cutoff values; $\mathrm{We}=141, \mathrm{Re}=\mathbf{5 7 0 0 ,} \mathrm{h} / \mathrm{d}=\mathbf{0 . 6 1 4}$.

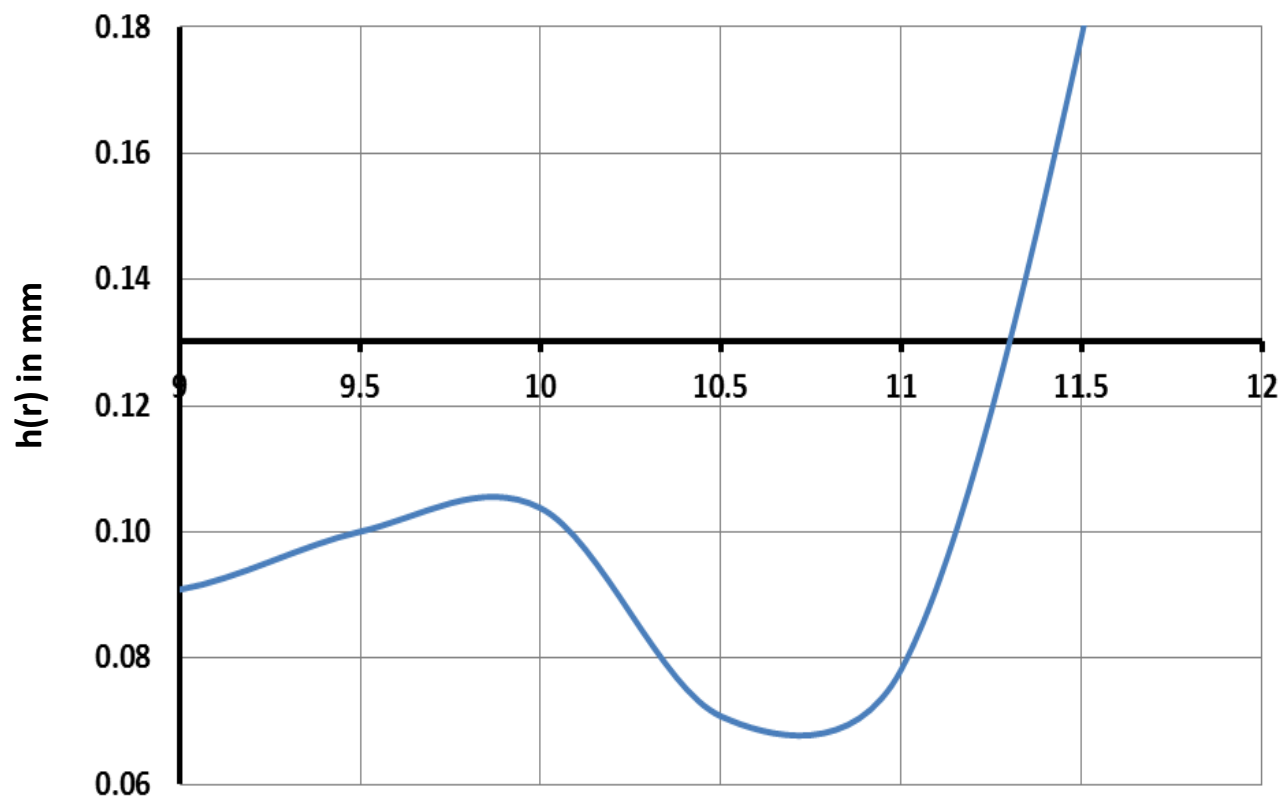

\section{Cavity radius in $\mathrm{mm}$}

Figure 6.2-4: Sulb-cavity liquid film thickness radial profille at $20 \mathrm{~ms}$ for $1.6 * \mathrm{~h} \_\mathrm{min}$, and horizontal axis is set at dimensional cutoff thickness value of $0.1302736 \mathrm{~mm}$; $\mathrm{We}=141, \mathrm{Re}=$ $5700, \mathrm{~h} / \mathrm{d}=0.614$. 


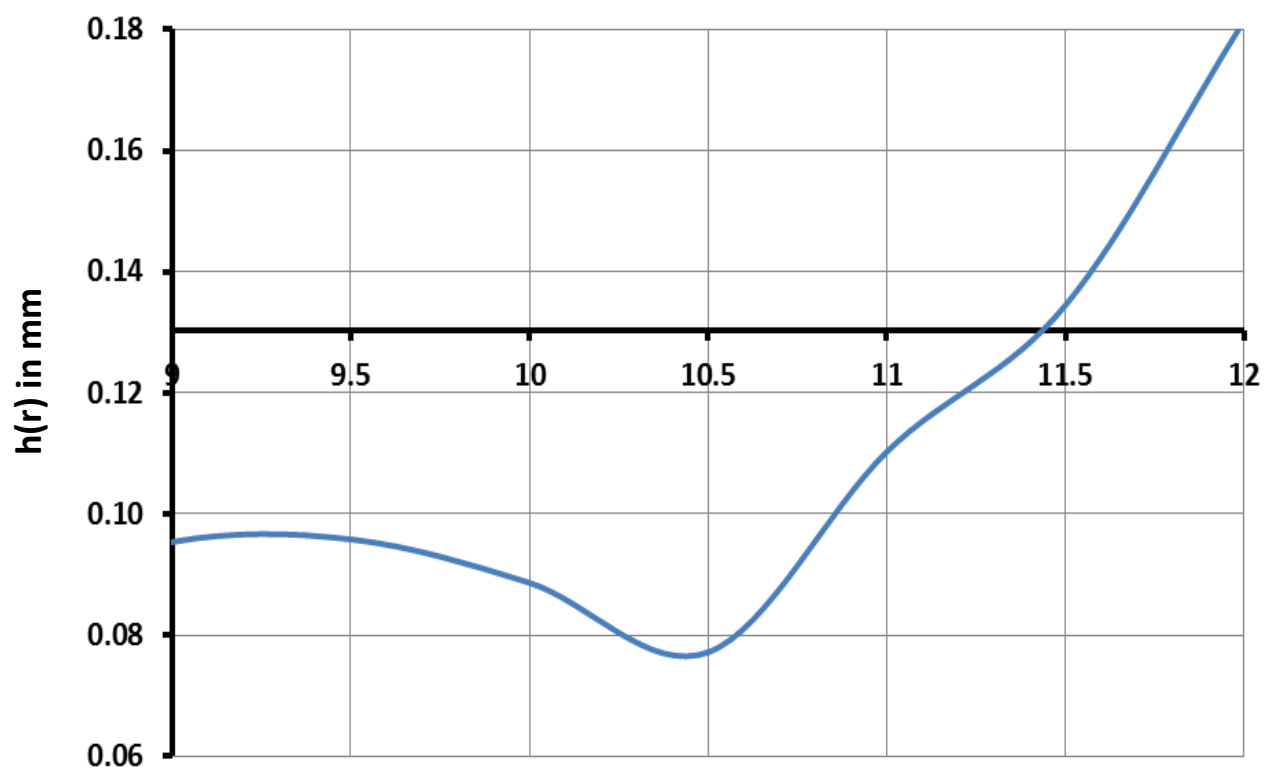

Cavity radius in $\mathrm{mm}$

Figure 6.2-5: Sub-cavity liquidd film thickness radial profile at $25 \mathrm{~ms}$ for $1.6 * \mathrm{~h} \_\mathrm{min}$, and horizontal axis is set at dimensional cutoff thickness value of $0.1302736 \mathrm{~mm}$; We $=141$, $\operatorname{Re}=5700, \mathrm{~h} / \mathrm{d}=0.614$.

Film thickness constraint values that are used for the calculation of sub-cavity volumes in experiments and in CFD simulations are listed in Table 6-1.

Table 6-1: Cut-off heights used for experiments and CFI simulations.

\begin{tabular}{|c|c|c|c|c|c|}
\hline & Experiment & \multicolumn{2}{|c|}{ 2D Fluent } & \multicolumn{2}{c|}{ 3D OpenFOAM } \\
\hline Case /Criterion & Running Average & $\begin{array}{c}\text { Constant } \\
\text { Cut-off }\end{array}$ & $\begin{array}{c}\text { Running } \\
\text { Average }\end{array}$ & $\begin{array}{c}\text { Constant } \\
\text { Cut-off }\end{array}$ & $\begin{array}{c}\text { Running } \\
\text { Average }\end{array}$ \\
\hline 1A & 193.5 & 240.8 & 295.2 & 224.5 & 208.6 \\
\hline 1C & 187.5 & 185.0 & 165.1 & 196.0 & 163.7 \\
\hline 3B & 140.0 & 135.3 & 138.3 & 133.2 & 126.2 \\
\hline 5A & 167.5 & 171.7 & 162.2 & 168.2 & 158.4 \\
\hline 5C & 122.0 & 129.4 & 126.1 & 125.0 & 125.4 \\
\hline
\end{tabular}




\section{Results}

\subsection{Overview}

This chapter discusses the results of the present work. When a drop impacts the free liquid surface, due to the momentum of the drop the static liquid is pushed downward creating a cavity until it reaches the impact surface. When it reaches the surface, a thin liquid layer is formed, termed the "subcavity liquid layer" in the current work. The thickness of this thin liquid layer is of interest, as a region of high transient heat flux in spray cooling applications (Kuhlman et al., 2007). After reaching the impact surface, the cavity starts to spread, thus increasing the cavity radius. This is continued until all the kinetic energy from the drop impact has been dissipated into the static liquid. This phase is called the cavity formation phase. In this phase, depending on the droplet Weber number, a crown is formed. At relatively low Weber numbers crown formation is not observed. The height of the crown, in the case of relatively high Weber number cases, is determined primarily by surface tension, and droplet impact velocity. The thickness of the thin liquid and the maximum cavity radius determine the maximum value of the sub-cavity volume. Calculating this volume quantitatively is the central objective of this thesis.

When the droplet kinetic energy has been completely dissipated into the surrounding liquid, the velocity of the cavity wall is decreased and soon comes to a complete stop. This distance from the center of the cavity to the cavity wall is termed as the maximum cavity radius. Now, the surrounding liquid starts exerting pressure on the cavity wall from outside. Also, surface tension pulls downwards on the crown liquid. This causes the thin liquid layer to rise and the shape of the cavity changes from a bowl to an inverted bell. Due to the liquid accelerating towards the center of the cavity, a Worthington jet often is formed.

These series of events are shown below in section 7.2 for both CFD simulations, compared with experiment, as a film strip of images arranged vertically. When the drop first comes into contact with the static liquid layer, that instant is considered as $0 \mathrm{sec}$. As the clock is started when the drop impacts the liquid layer, it is termed as the impact time. The elapsed time when the centerline sub-cavity film thickness first begins to increase again is defined as the total cavity lifetime. Section 7.3 presents the 
CFD results for the sub-cavity liquid film thickness. These results are also compared with the experiments by Hillen (2013). Finally, the computed sub-cavity volumes are compared with these experiments in section 7.4. Note that for the $2 \mathrm{D}$ axisymmetric simulations there were between 4 and 8 mesh points within the sub-cavity film thickness, and between 6 to 10 mesh points for the 3D simulations.

\subsection{Comparisons of CFD simulations and Experiments}

In this section, side by side image comparisons are made for 2D axisymmetric ANSYS Fluent, 3D OpenFOAM, and experiment cases. This type of comparison gives one an initial qualitative idea about the re-creation of the experiments in a virtual world. The images in this section for 2D axisymmetric ANSYS Fluent are the mirror image of the simulation along the axis, while for 3D OpenFOAM it's the full cavity viewed from the front of the domain and the same is for experiment. By varying the Weber number and $h^{*}$, we observe how the behavior the cavity, crown, and splashing phenomenon change dramatically. For higher Weber number cases, the cavity lifetime is observed to be significantly higher than the cases with lower Weber number.

In Figure 7.2-1, a sequence of images, organized vertically like film strips, are shown with their respective impact times displayed in red. The experimental images have been obtained from the videos within the thesis by Hillen, (2013). For the images to be compared, a common procedure was setup. For that reason, a specific methodology was developed to obtain these sequences of images. The first image is taken at an impact time of 0 sec; i.e., when the drop just touches the surface of the static liquid layer. The second image has been selected at the instant when the cavity is fully established and the centerline film thickness gets to its minimum value. The third and fourth images will be explained after the fifth image. The fifth image is selected at the instant when the centerline film thickness begins to increase and the last calculation of the sub-cavity volume can be obtained. The third and fourth images are taken at 50\% and 75\% of the timespan between the second and fifth images. 


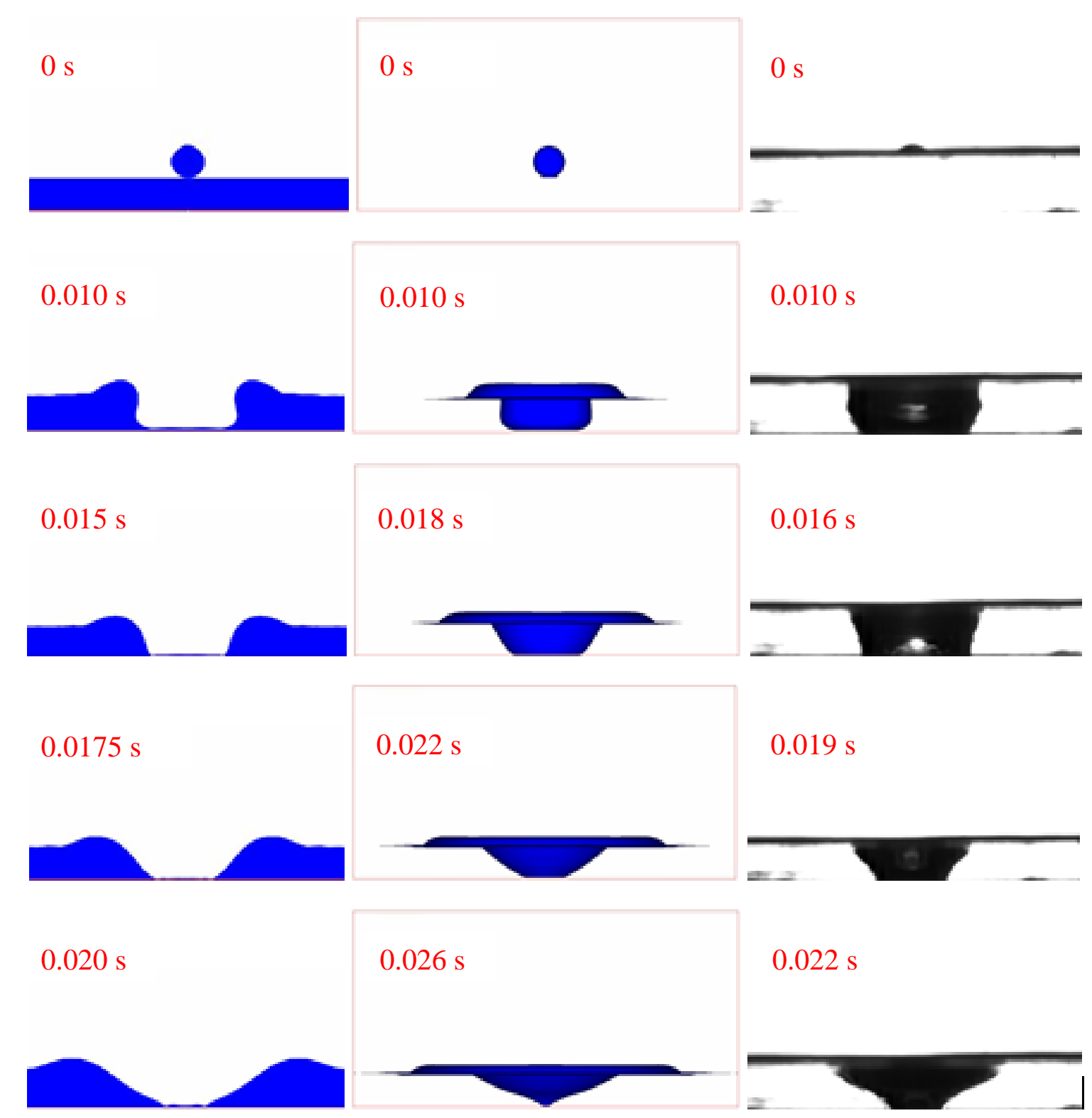

Figure 7.2-1: Sequence of images for the case $1 \mathrm{~A}(\mathrm{We}=145, \mathrm{Re}=1189, \mathrm{~h} / \mathrm{d}=1.0)$ : First column is 2D Axisymmetric Fluent, second column is 3D OpenFOAM and third column is experiment.

From the first image of the experiment, it appears as if the drop has almost disappeared into the static liquid layer, but it's just grazing the surface of the liquid layer. This is a result of the perspective view due to the position of the camera, as indicated schematically in Figure 7.2-2. The camera lens was aligned parallel to the bottom impact surface to capture the exact shape of the cavity rather than the crown and splashing. Due to the limitations of the camera used, the distance from the experiment apparatus is short. 


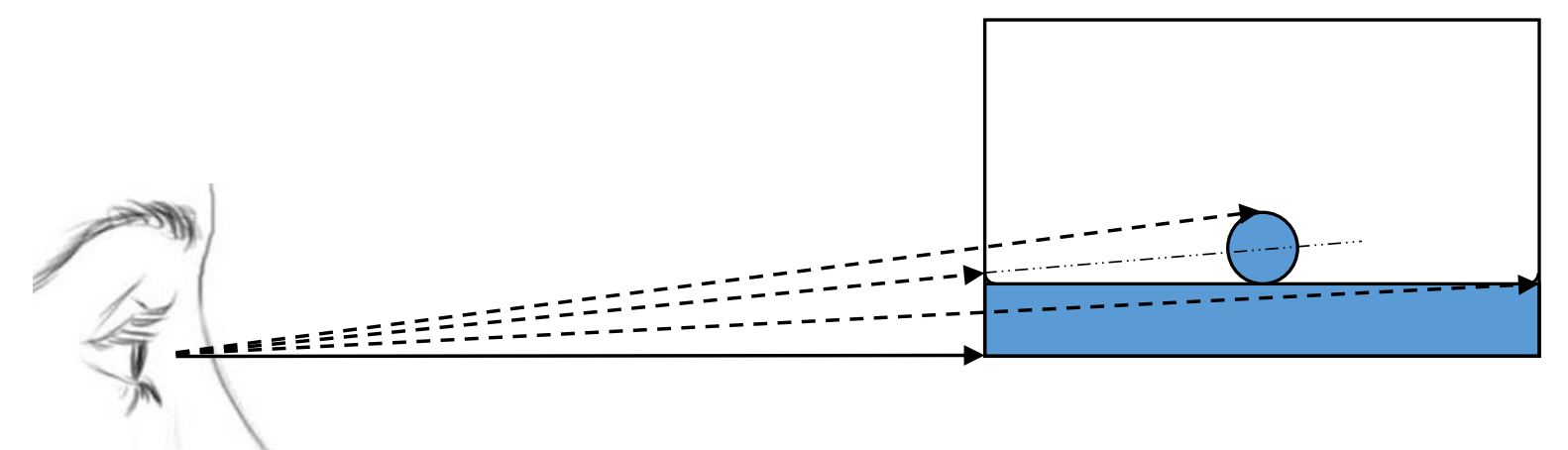

Figure 7.2-2: Representation of the perspective view while capturing the videos for the experiments performed by Hillen in Hillen, 2013. Not to scale.

In Figure 7.2-2, the human eye represents the camera position. The solid straight, horizontal line is the camera's line of sight, aligned to the impact surface. The two dashed lines represent the field of view through which we are able to see the drop and the free surface of the liquid layer. The extended long-dash-dot-dot line, cuts through the drop at the top the near wall meniscus. The portion of the drop which is below this line cannot be seen in the videos. This is the reason that much of the drop is not visible in the first image of the experiment column in Figure 7.2-1. Due to the refraction of light when it is passing through the water and glass, the light bends and block more of the drop from the view. A meniscus also obstructs the light which is coming from the wall away from the camera. A meniscus is present on both the front and rear walls of the experiment.

From the sequence of images in Figure 7.2-1, it can be seen that the CFD simulations are generally in good agreement with the experiments and with each other, for this low We case. During the cavity formation phase, the first two images are captured at the same times in all the three columns. It is during the retraction phase, where the times diverge from one another. In the second and third images of the first and second columns, the cavity shape is retained which is slightly different from the same images in third column. Due to the axisymmetric nature of the 2D simulations, the cavity starts filling in somewhat more quickly compared to both the 3D OpenFOAM simulations and the experiments.

Berberovic et al. (2009), observed a wave projecting downward on the inner wall of the cavity starting from the top of the cavity running towards the impact surface. This was seen only during the retraction phase. This action was called in that paper a "capillary wave", but Hillen, (2013) described 
it as a "cavity wave", as that wave is also partially driven by gravity. However, this type of behavior is not observed in either of the CFD simulations for this case.

Figure 7.2-3 gives the times at which the drop just touches the liquid layer, first sub-cavity volume calculation, half and $3 / 4^{\text {th }}$ of the time between first and last sub-cavity volume calculation, and last sub-cavity volume calculation of the case $1 \mathrm{C}$, again at a low We of 135 , but here with $\mathrm{h} / \mathrm{d}=0.2$. The liquid layer is so thin that it is difficult to observe the sub-cavity region from these images. Hence, a zoomed strip of images of the $2 \mathrm{D}$ axisymmetric fluent simulation has been shown for each time in Figure 7.2-4.

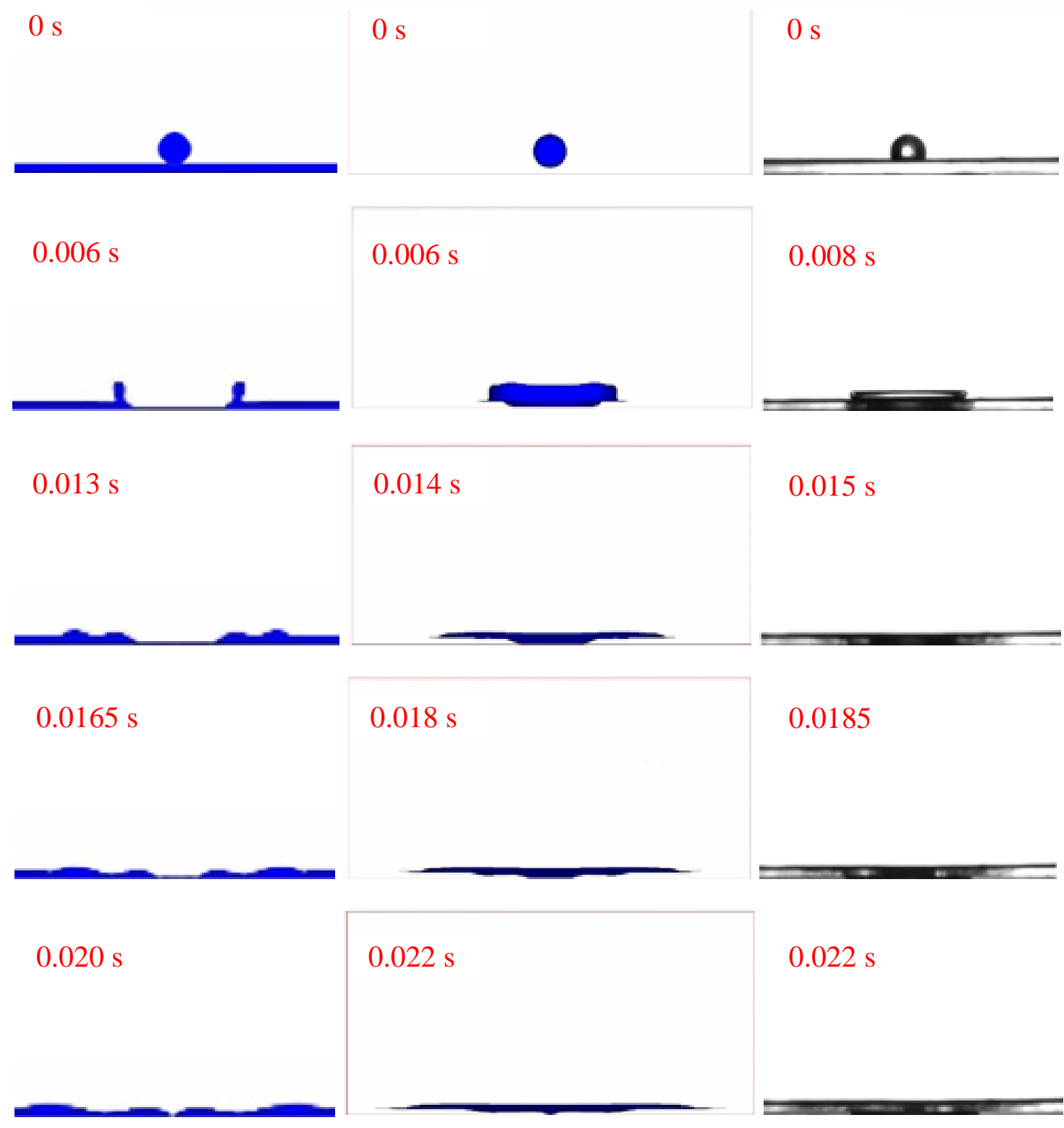

Figure 7.2-3: Sequence of images for the case $1 \mathrm{C}(\mathrm{We}=135, \mathrm{Re}=1176, \mathrm{~h} / \mathrm{d}=\mathbf{0 . 2})$ : First column is 2D Axisymmetric Fluent, second column is 3D OpenFOAM and third column is experiment. 
Since the height of the static liquid layer is $1 / 5^{\text {th }}$ of the drop diameter, much more of the drop liquid is visible at 0 seconds for the experiment. During the cavity formation phase, it can be observed that 2D and 3D simulations agree with each other but both are offset somewhat from the experiment. Figure 7.2-5 shows the zoomed images for the 3D OpenFOAM simulations, and Figure 7.2-6 presents the

$0 \mathrm{~s}$

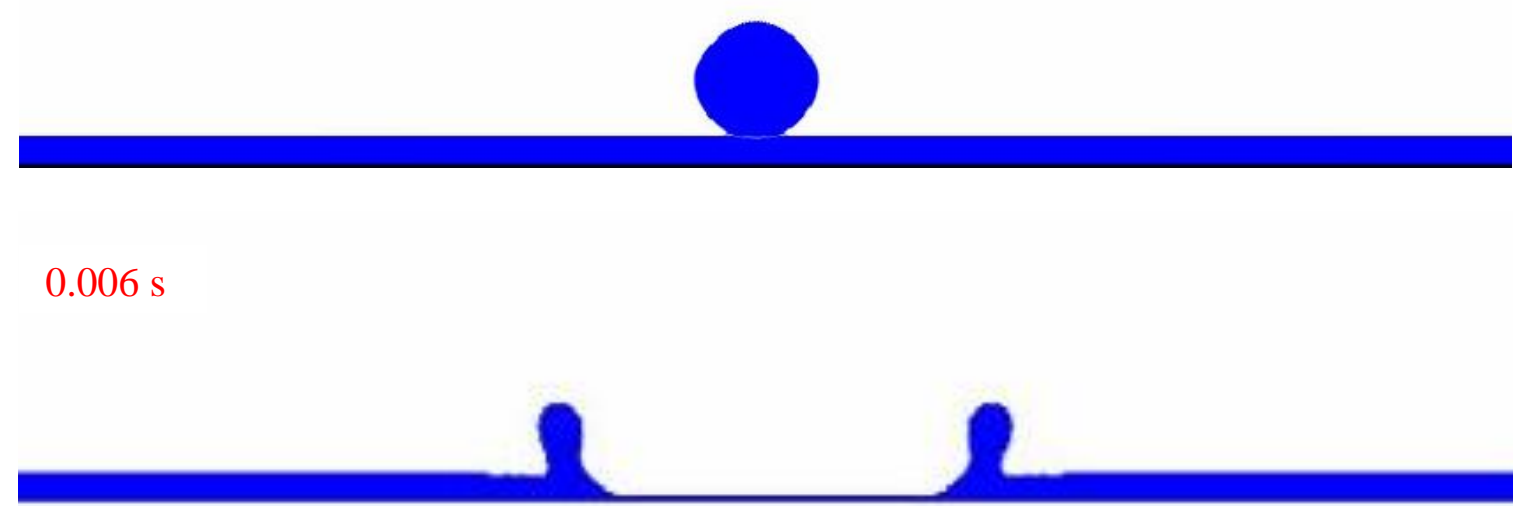

$0.013 \mathrm{~s}$

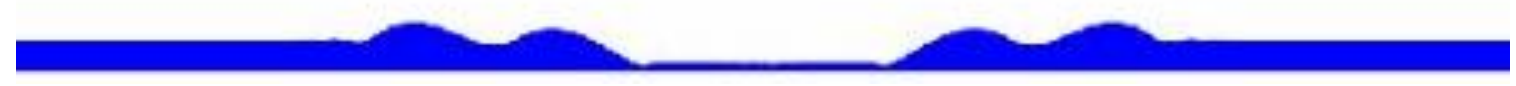

$0.0165 \mathrm{~s}$

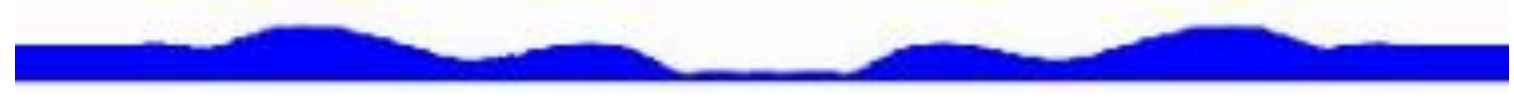

$0.020 \mathrm{~s}$

Figure 7.2-4: Zoomed sequence of images for 2D axisymmetric Fluent simulation of case 1C. corresponding zoomed images for the experiment. During the retraction phase, it can be seen that the 3D simulation is excellent in predicting the cavity lifetime. In the formation phase, the crown of the experiment is flat, while the $3 \mathrm{D}$ simulation image shows tiny bumps on its crown. When observed 
from the top, these bumps are pointed along the diagonals of the square calculation domain. Due to the slight imbalance of mass flux through the corner points and the side faces of the cell corresponding to that corner point, this anomaly is observed. As the simulation proceeds, this error is more prominently observed in the cells that are larger than the cells near to the impact surface. In order to avoid this, a cylindrical domain should be used which would eliminate the corners. This assures that the sub-cavity region is circular, as expected, and is simulated correctly.

$0 \mathrm{~s}$

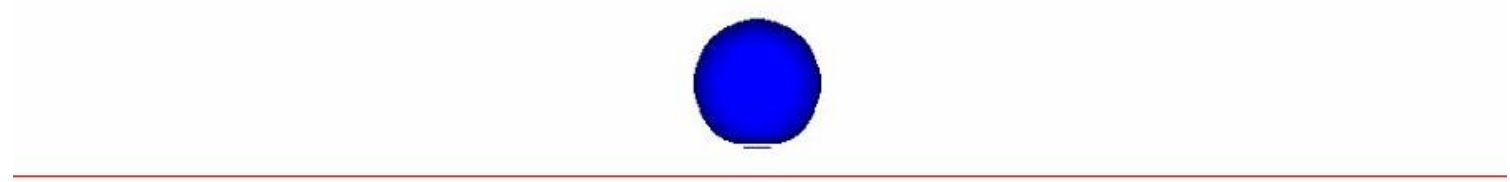

$0.006 \mathrm{~s}$

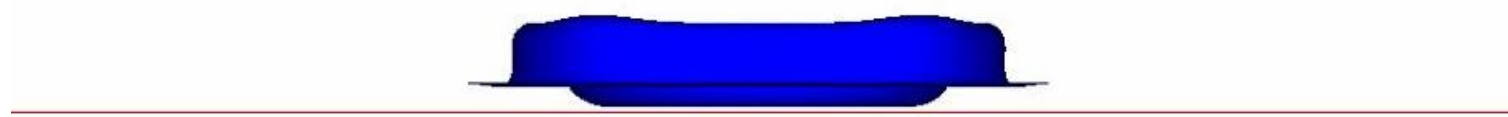

$0.014 \mathrm{~s}$

$0.018 \mathrm{~s}$

$0.022 \mathrm{~s}$

Figure 7.2-5: Zoomed sequence of images for 3D OpenFOAM simulation of case 1C. 
To see the sub-cavity region more clearly, the experiment videos have also been zoomed to the same extent as the 2D and 3D simulation images. From the images of experiments in Figure 7.2-6, it can be observed that the sub-cavity region is not clearly distinguishable from the surrounding liquid. This is due to the refraction of light through the thin liquid layer, and the disturbances caused due to the crown collapse and cavity fill in. Thus, not enough light is propagated between the (nearly) parallel liquid free surface and the bottom wall. For this type of case, centerline film thickness and the subcavity volumes as a function of time are more difficult to compare with the experiments.

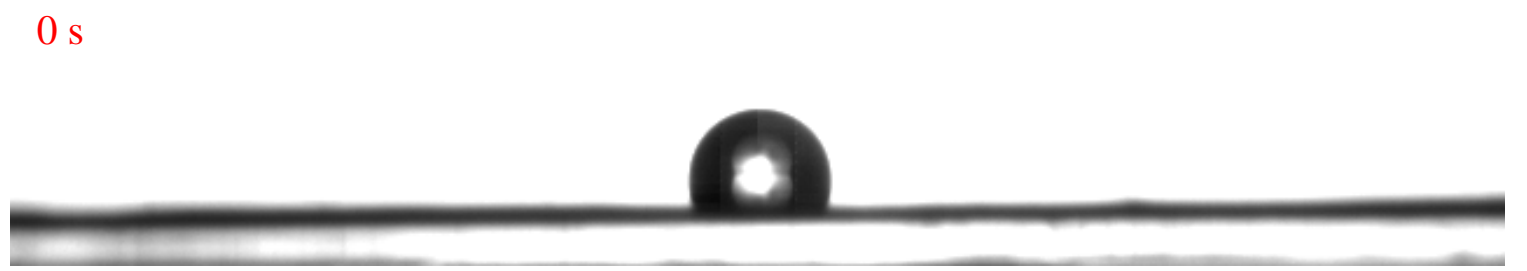

$0.008 \mathrm{~s}$

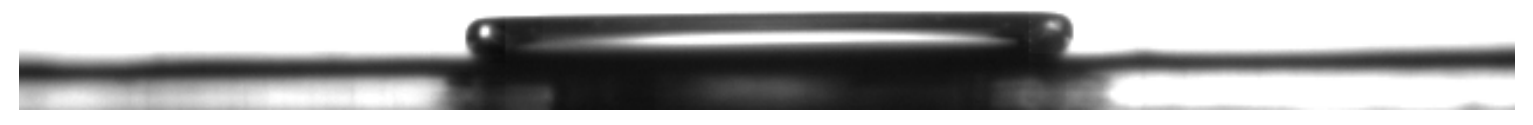

$0.015 \mathrm{~s}$

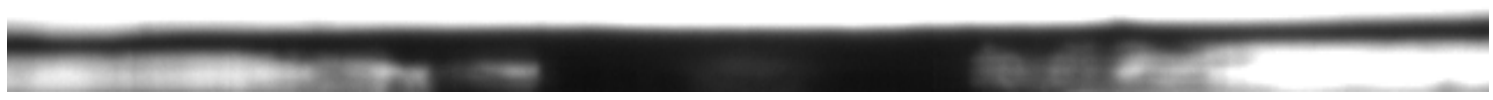

$0.0185 \mathrm{~s}$

$0.022 \mathrm{~s}$

Figure 7.2-6: Zoomed sequence of images for experiment of case 1C (Hillen, 2013). 
In Figure 7.2-7, the images for case $3 \mathrm{~B}(\mathrm{We}=621, \mathrm{Re}=2643$, and $\mathrm{h} / \mathrm{d}=0.5)$ are shown. As $\mathrm{We}$ is increased, it takes more time for the cavity to fill in, and as a result the cavity lifetime is increased. From the images of Figure 7.2-7, it can be seen that there is no secondary splashing involved in the experiment. The same is observed in the $2 \mathrm{D}$ Fluent simulation. When there is no discontinuity in the crown, the surface tension is uniform and holds the crown liquid with equal force around the circumference. A similar behavior is observed in experiment and the 2D Fluent simulation. However, in the 3D OpenFOAM CFD solution breakup of the crown is predicted and the crown liquid forms into droplets (secondary splashing), which delays the cavity fill in. This is believed to be the reason for the extended cavity lifetime predicted in the 3D simulations.

A capillary wave on the inner wall of the cavity can be observed in the experiment at $21 \mathrm{~ms}$ whereas it just starts to form in the 2D Fluent simulation at the same time. From the images, it is visible that the size of the cavity in the 2D Fluent simulation matches well with that of the experiment. The reason that the 3D OpenFOAM solution predicts onset of secondary splashing when none is observed in experiments for this case is not known, although it may be due to the computational mesh not being fine enough near the crown. A mesh adaption method in OpenFOAM requires higher computational resources than we have presently; this would be expected to improve the $3 \mathrm{D}$ simulations when the resolution of the mesh is increased at the air-liquid interface. 


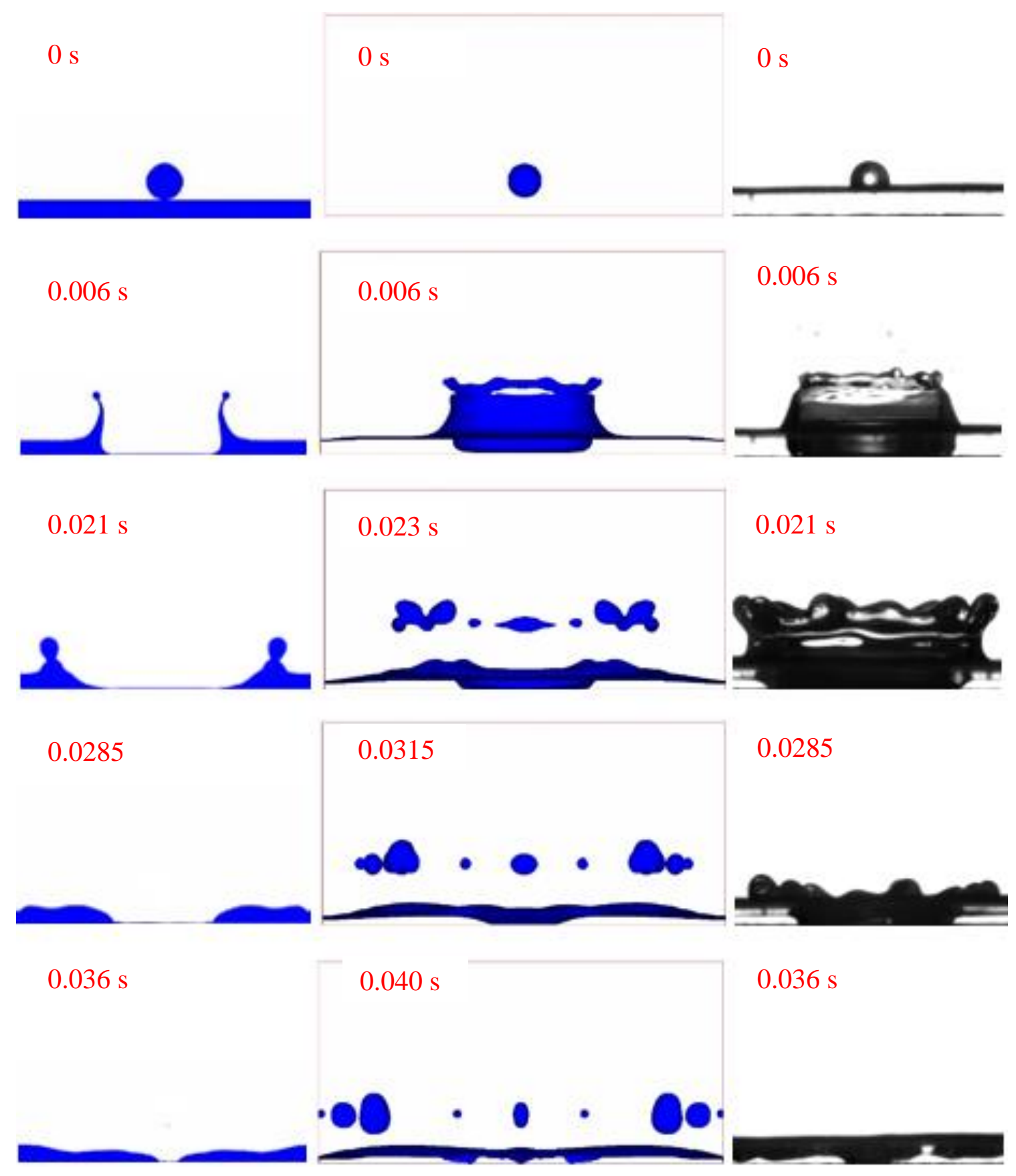

Figure 7.2-7: Sequence of images for the case $3 \mathrm{~B}(\mathrm{We}=621, \mathrm{Re}=2643$, and $\mathrm{h} / \mathrm{d}=0.5)$ : First column is 2D Axisymmetric Fluent, second column is 3D OpenFOAM and third column is experiment.

In Figure 7.2-8, the series of images of 2D Fluent, 3D OpenFOAM, and experiment drop impacts for case $5 \mathrm{~A}(\mathrm{We}=993, \mathrm{Re}=3574$, and $\mathrm{h} / \mathrm{d}=0.9)$ are shown. In the second row of images, it can be seen that the shape of the crown and cavity radius of $2 \mathrm{D}$ and $3 \mathrm{D}$ simulations match well with that of experiment. There is no secondary splashing involved in this thick layer of liquid, high-We number case. Although there are a few blobs of liquid observed on top of the crown in the third row 
(experiment) of images, there is no evidence of droplet formation. Capillary waves are propagating down the inner side of the crown wall. Since, the images are captured at regular percentage intervals of time depending on the cavity lifetime, the times at which the events occur are varied but the cavity radius is approximately the same in the CFD simulations and experiment at corresponding percentages of the cavity lifetime.

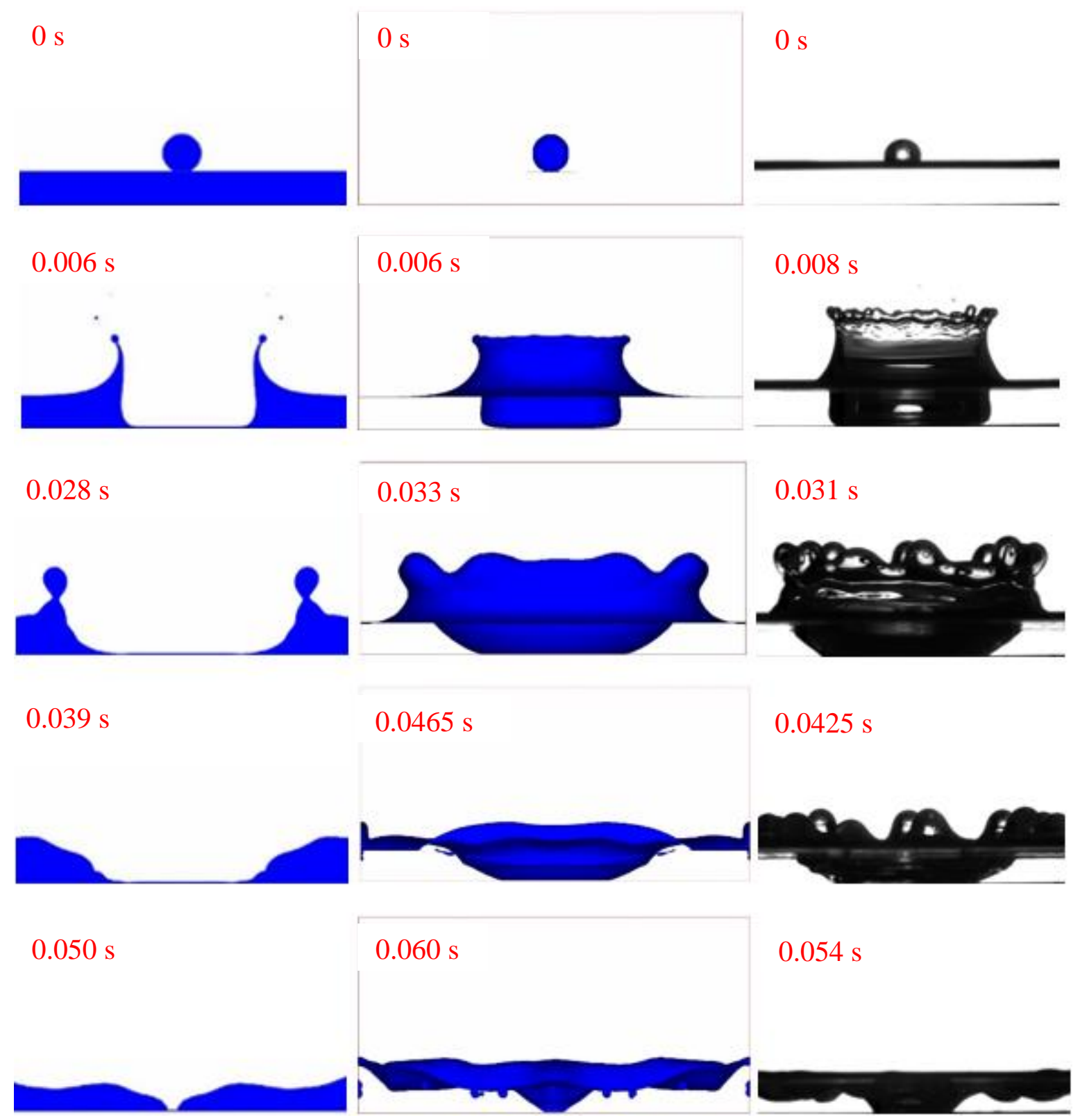

Figure 7.2-8: Sequence of images for the case $5 \mathrm{~A}(\mathrm{We}=993, \mathrm{Re}=3574$, and $\mathrm{h} / \mathrm{d}=0.9)$ : First column is 2D Axisymmetric Fluent, second column is 3D OpenFOAM and third column is experiment. 
Case $5 \mathrm{C}(\mathrm{We}=984, \mathrm{Re}=3555$, and $\mathrm{h} / \mathrm{d}=0.2)$ is very different in the CFD simulations compared to the experiment. In Figure 7.2-9, the three series of images for case 5C are presented. It is clearly evident that the formation of the cavity, collapse of the crown, and cavity fill-in are each entirely different in both of the CFD simulations than in the experiments. The crown in the experiments behaves more like an elastic membrane or surface, which is stretched due to the momentum of droplet impact but is restricted by surface tension. When the momentum has been dissipated, the surface

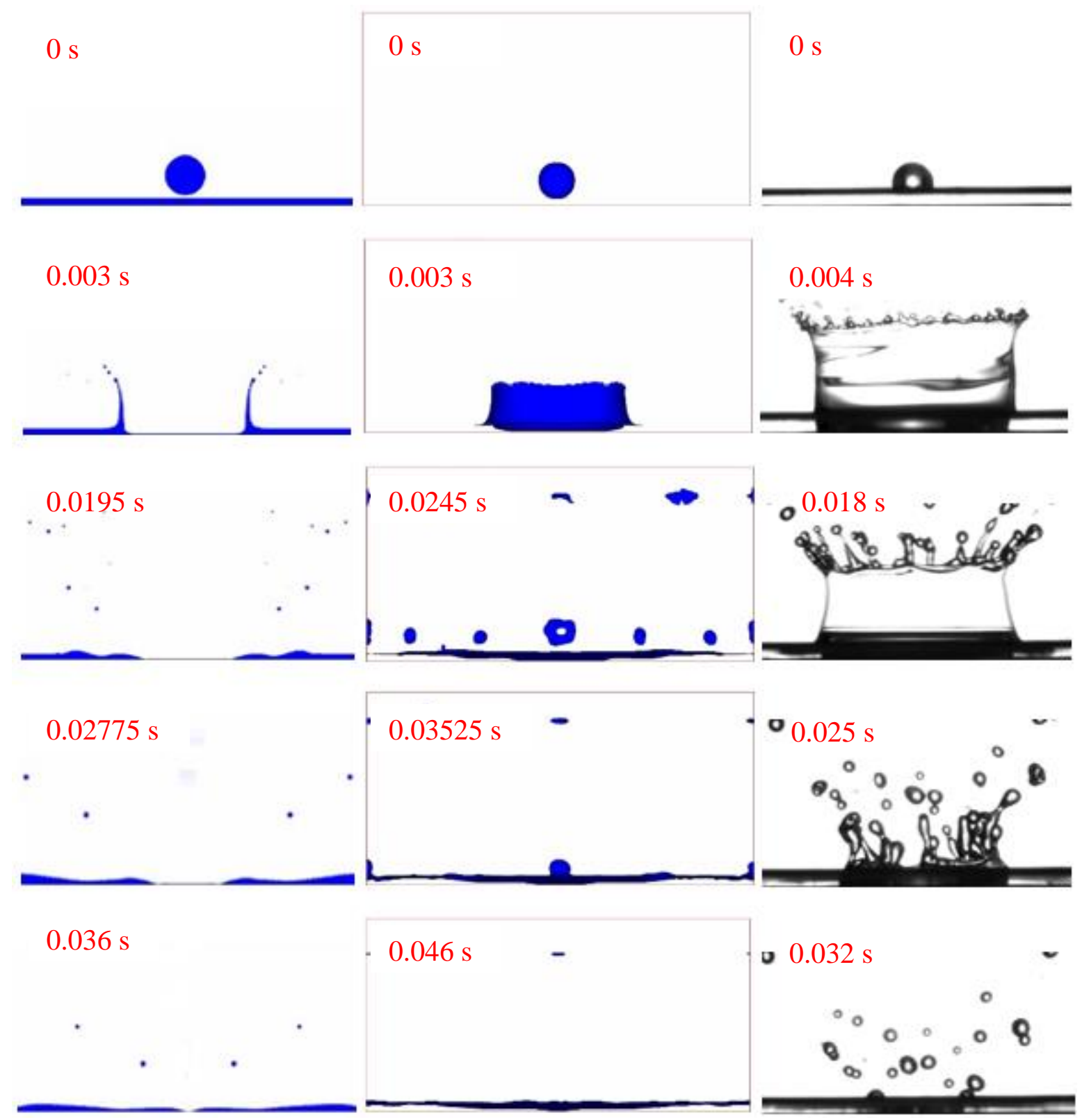

Figure 7.2-9: Sequence of images for the case $5 \mathrm{C}(\mathrm{We}=984, \mathrm{Re}=3555$, and $\mathrm{h} / \mathrm{d}=0.2)$ : First column is 2D Axisymmetric Fluent, second column is 3D OpenFOAM and third column is experiment. 
tension comes into play and contracts and pulls the crown downwards and towards the center of the cavity. All the liquid from the crown splashes into the cavity, forcing the cavity to fill in more quickly. This is the reason for the early collapse of the cavity, which is evident in the Figure 7.2-9. Whereas in CFD simulations, the crown breaks into rings in the 2D Fluent simulation, and into secondary splashing in the 3D OpenFOAM simulation very early during the retraction phase, leading to longer predicted cavity fill-in times.

Most of the liquid in the crown of the 3D simulation is dispersed into air and so there is no weight and a reduced surface tension force in the crown to force the cavity to fill in. Hence, the 3D cavity fill-in takes longer than the 2D and experiment cavity fill-in times. Because of the shallow liquid layer on the bottom wall, the cavity is not clearly visible. This may be seen more clearly in the following series of images, Figure 7.2-10 through Figure 7.2-12, which show the zoomed views of the 2D and 3D simulations and experiment, respectively. Here again it is somewhat difficult to see the details of the cavity fill in for the experiment in Figure 7.2-12. 


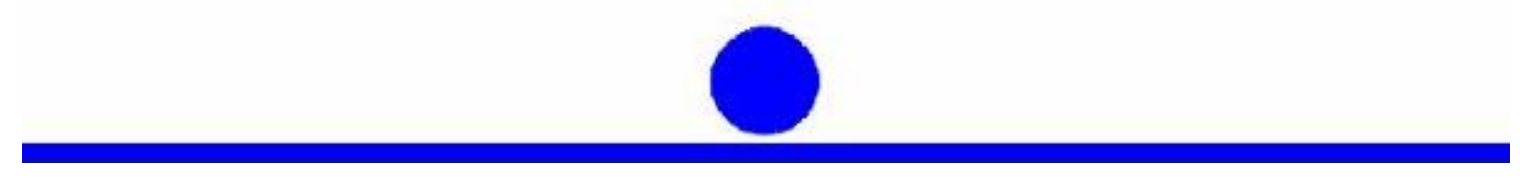

$0.003 \mathrm{~s}$
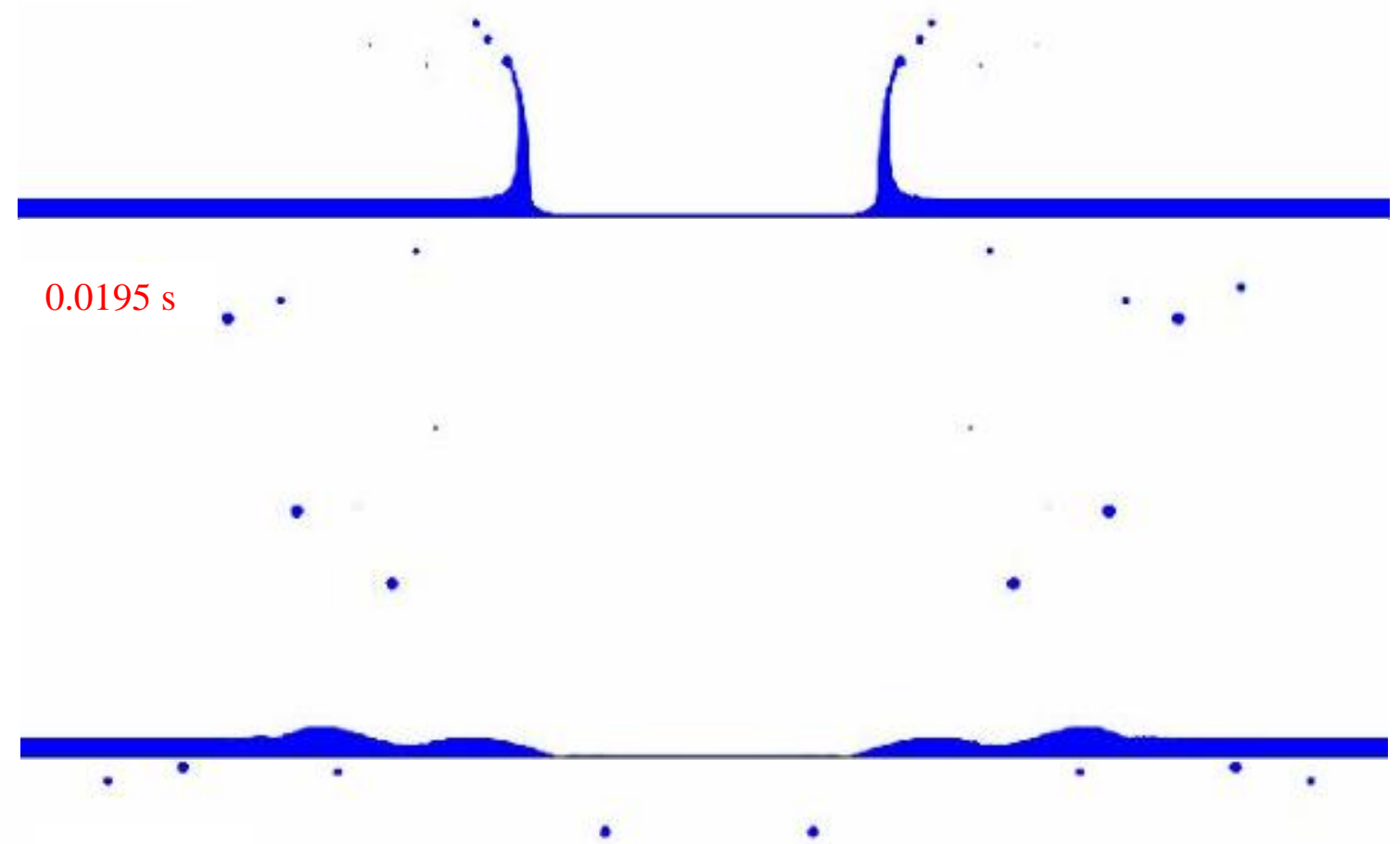

$0.02775 \mathrm{~s}$

-

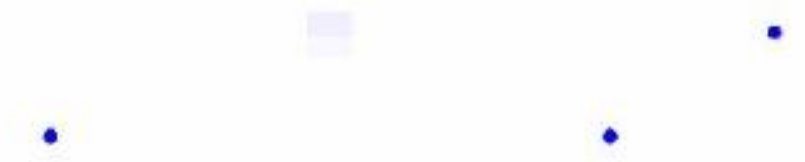

$0.036 \mathrm{~s}$

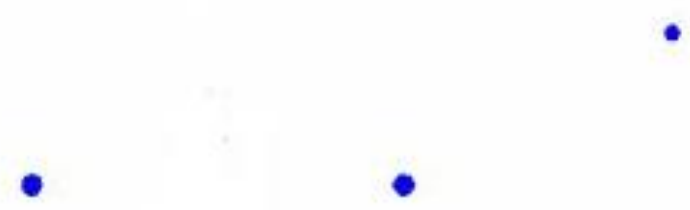

Figure 7.2-10: Zoomed sequence of images for 2D axisymmetric Fluent simulation of case 5C. 


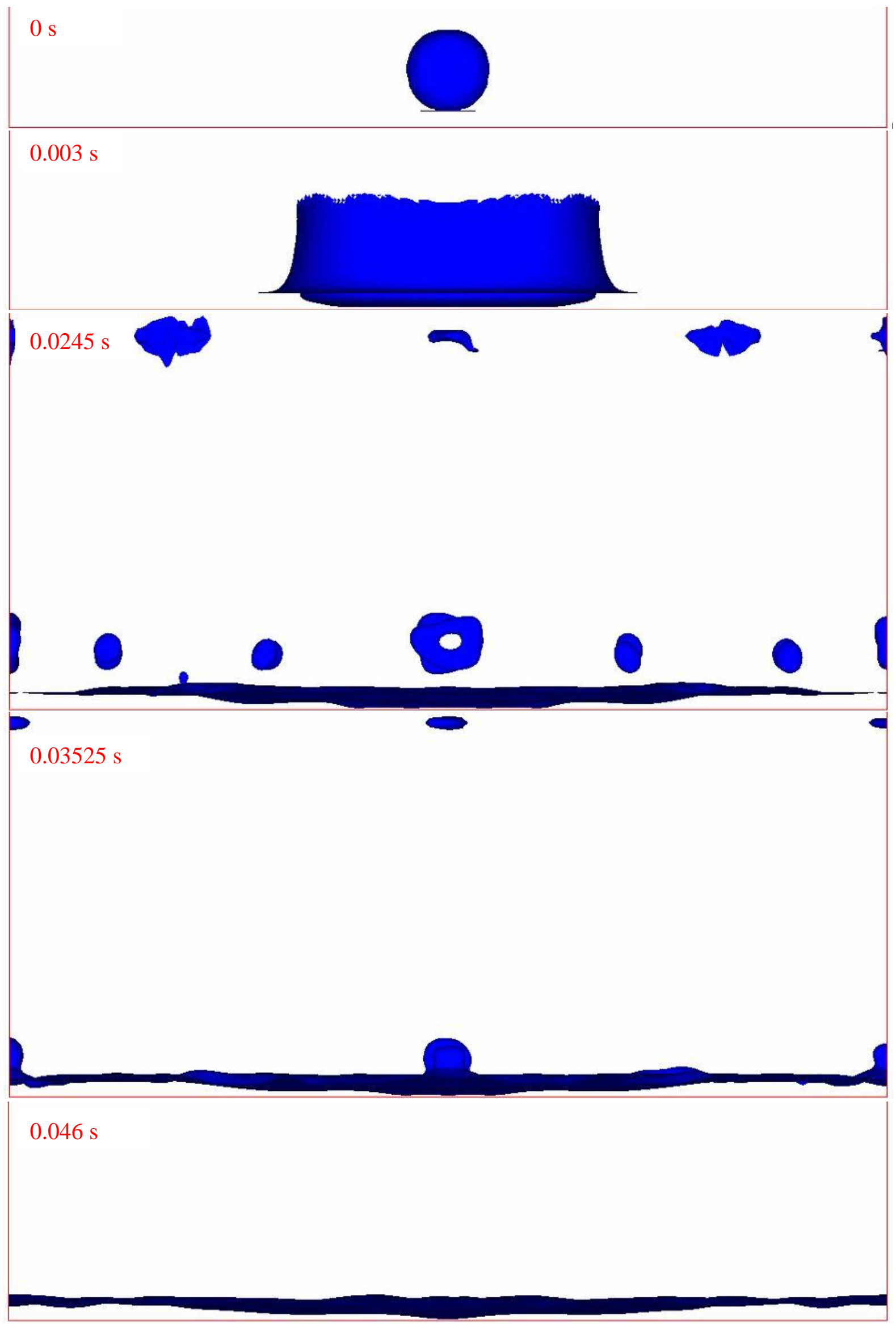

Figure 7.2-11: Zoomed sequence of images for 3D OpenFOAM simulation of case 5C. 

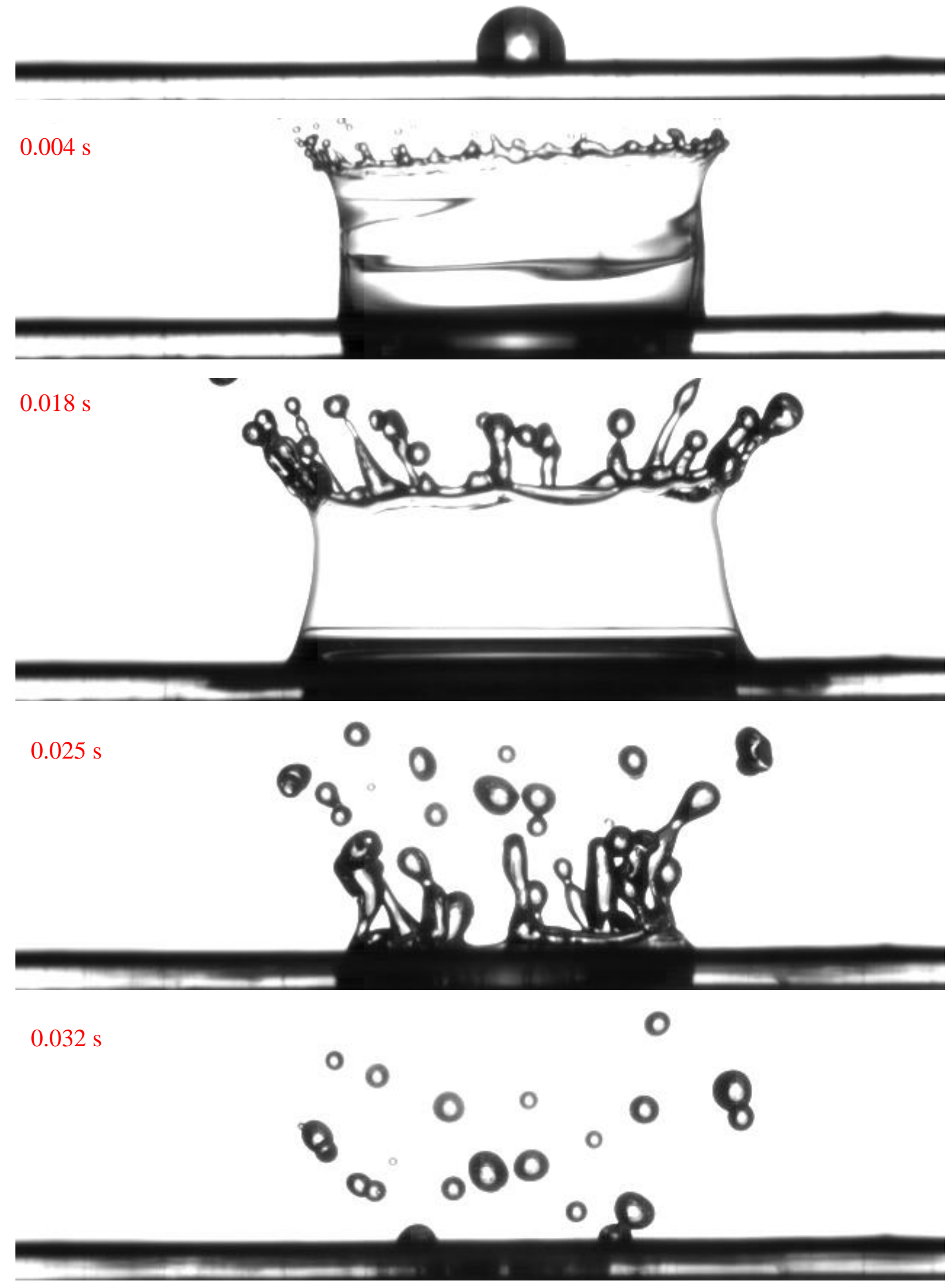

Figure 7.2-12: Zoomed sequence of images for experiment of case 5C (Hillen, 2013). 


\subsection{Comparison of Centerline Film Thickness}

After comparing the visual aspects of the simulations with experiment, the next thing that was compared was the centerline film thickness. The centerline film thickness is the height of the liquid above the bottom wall at the center of the cavity region. This centerline film thickness gives the information about cavity lifetime and the formation of any Worthington jet. This section will discuss the cavity centerline thickness histories of both CFD simulations and compare them with that of the experiment. The normalized centerline film thickness is calculated by:

$$
h^{*}=h / d
$$

The non-dimensional time is calculated as:

$$
t^{*}=t \frac{V e l}{d}
$$

Figure 7.3-1 shows the centerline film thickness profiles of 2D ANSYS Fluent, 3D OpenFOAM, and experiment for the case $1 \mathrm{~A}$. It is observed that in the initial cavity formation phase, all the three profiles nearly overlap each other indicating that the CFD simulations predict the centerline film thickness very well during early times. At $t^{*}=0$, the $h^{*}=1$ for experiments whereas it is 2 for CFD simulations. This is because in the CFD simulations even the drop diameter is included in the

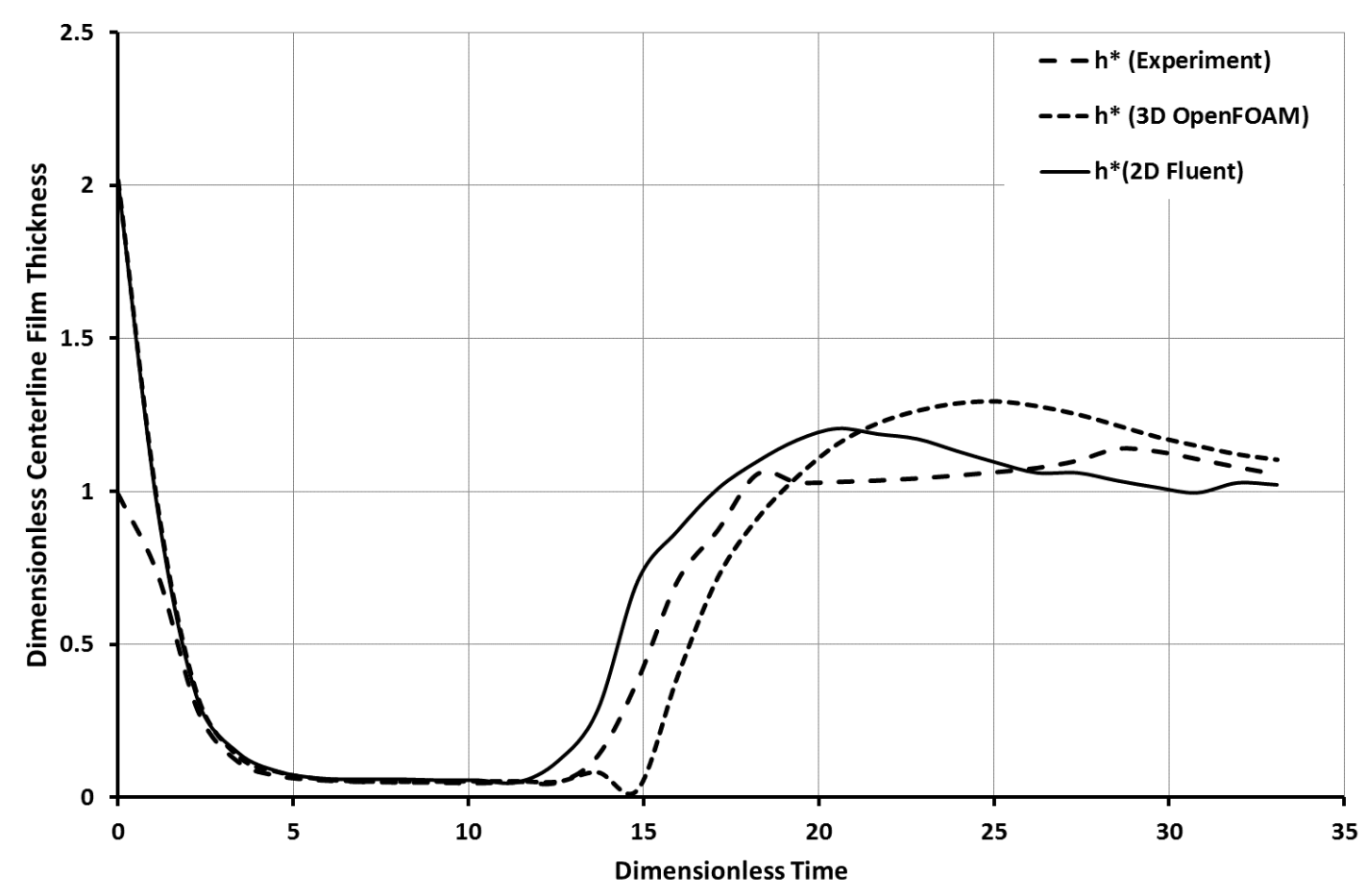

Figure 7.3-1: $h *$ vs $t^{*}$ plot for case $1 \mathrm{~A}(\mathrm{We}=145, \operatorname{Re}=1189, \mathrm{~h} / \mathrm{d}=1.0)$. 
calculations. However, during the retraction phase the 2D Fluent solution shows an early cavity fill-in compared to that of the experiment, whereas the 3D OpenFOAM solution overshoots the experiment cavity lifetime. Cavity waves are seen in the 3D OpenFOAM solution time histories while those are not seen in the 2D Fluent solution and in the experiment. It is speculated that due to the variation in surface tension of the liquid used in the experiment, because of the laboratory environment, the cavity lifetime is shorter and the maximum height reached by the Worthington jet is also less compared to the 3D OpenFOAM, where the surface tension is constant throughout the simulation. The percentage change in cavity fill-in times will be discussed quantitatively in section 7.4.

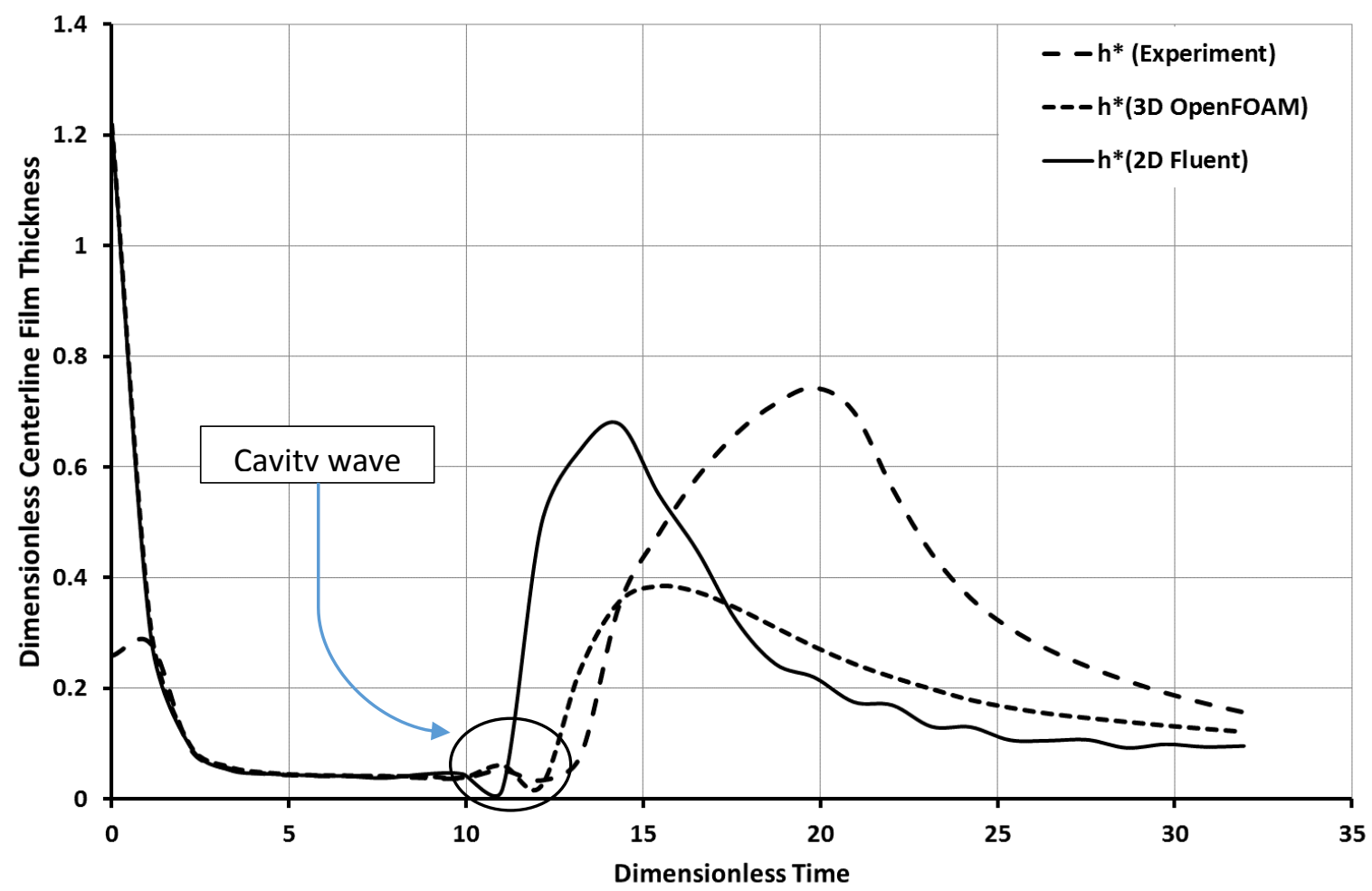

Figure 7.3-2: $h *$ vs $t *$ plot for case $1 \mathrm{C}(\mathrm{We}=135, \mathrm{Re}=1176, \mathrm{~h} / \mathrm{d}=0.2)$.

Centerline film thickness time histories for case 1C are shown in Figure 7.3-2. In the initial stages of the drop impact, for CFD simulations we see that the height of the centerline film is much higher than that of the experiment. This is because in the CFD simulations the initial height of the centerline film is a combination of drop diameter and the initial static liquid film height, whereas in the experiment it is defined as just the static liquid film height. In the cavity formation phase, the CFD solutions exactly predict the behavior of the centerline film thickness when compared with that of the experiment. The cavity waves can also be seen at the cavity fill-in time, i.e., retraction phase in all the three curves. 
The 2D Fluent solution predicts a faster cavity fill-in than the experiment. For this case, we can observe that the height of the Worthington jet for the experiment is higher than the CFD solutions predict and also the cavity fill-in time is greater.

In Figure 7.3-3, the centerline film thickness time histories of both CFD simulations and the experiment for case 3B are shown. As discussed for the case 1C, in the initial stages of impact, the centerline film thickness is calculated including the drop diameter in CFD simulations which was not done in case of experiment. In this case, we observe a centerline film thickness time history makes an angle slightly greater than $90^{\circ}$. This sharp profile is formed due to an increase in the droplet Re. The velocity of the drop is $3.546 \mathrm{~m} / \mathrm{sec}$ which is bigger than $1.727 \mathrm{~m} / \mathrm{sec}$ and $1.667 \mathrm{~m} / \mathrm{sec}$ for case $1 \mathrm{~A}$ and $1 \mathrm{C}$, respectively. If the profiles are compared with the profiles of the previous cases, we also notice that the centerline film thickness is less when the cavity has been fully established. As expected, the 2D Fluent simulation predicted an early cavity fill-in during the retraction phase. Though the CFD simulations predict the cavity formation and the centerline film thickness accurately, the retraction phase is always different between the CFD solutions and experiment. There are no cavity waves

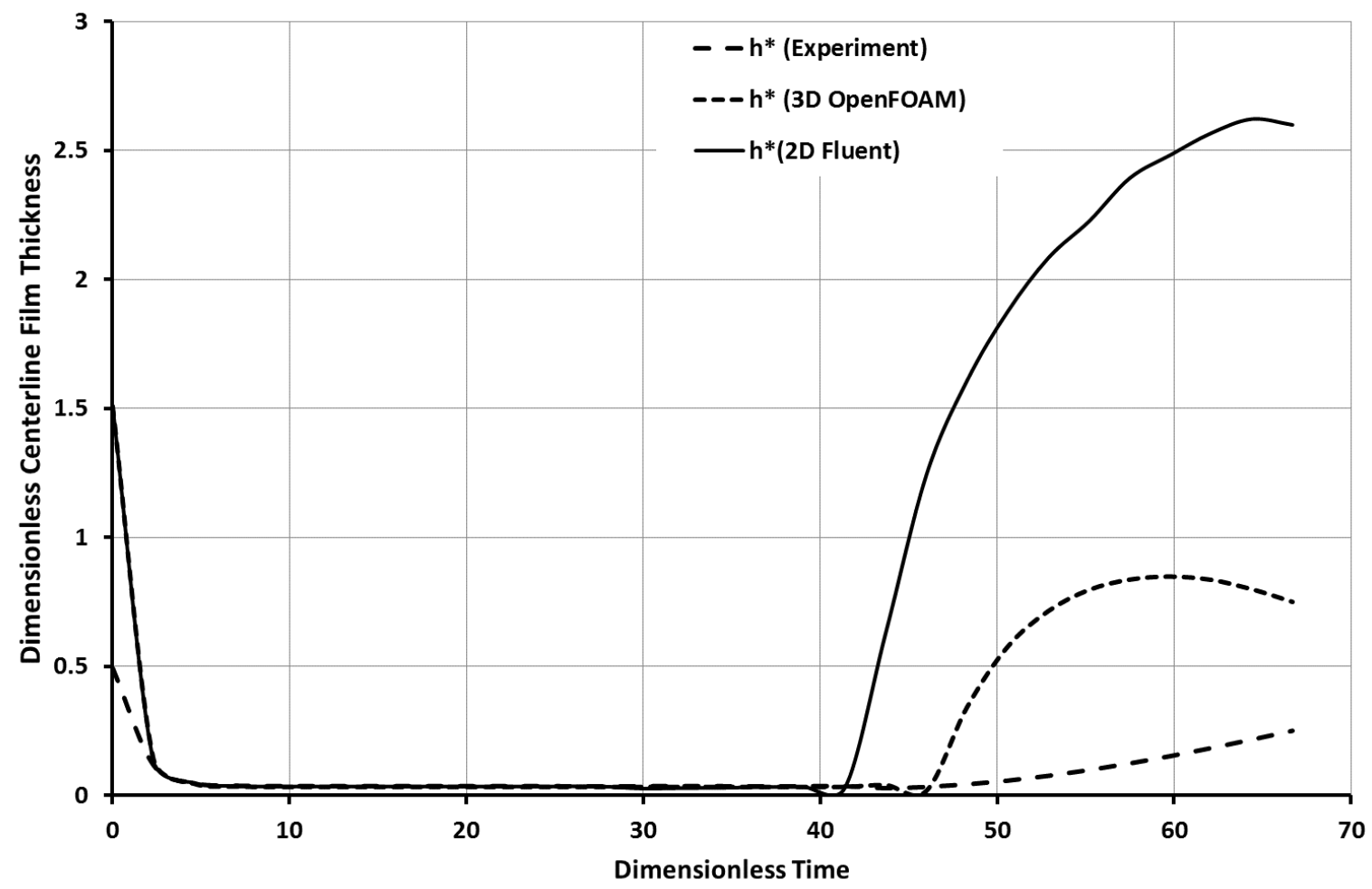

Figure 7.3-3: $h *$ vs $t *$ plot for case $3 \mathrm{~B}(\mathrm{We}=621, \mathrm{Re}=2643$, and $\mathrm{h} / \mathrm{d}=0.5)$. 
observed in this case. A fillet (See pages 36-41 for discussion of the term, "fillet", and the effects of these fillets on the computation of sub-cavity volumes.) in the profile just before the start of the Worthington jet is seen in the CFD simulations.

From the strip of images showed for case 3B (Figure 7.2-7), it can be noticed that there is no splashing in the 2D Fluent solution whereas splashing is observed in 3D OpenFOAM solution and this causes some of the liquid to escape and loss of momentum required for cavity fill-in. The experiment takes more time for the cavity to fill-in, although both the 2D Fluent simulation and the experiment start the retraction phase at the same time.

Figure 7.3-4 shows the centerline film thickness time histories of both CFD simulations and experiment for case 5A. The velocity of the impacting droplet is $4.196 \mathrm{~m} / \mathrm{sec}$. Due to the higher velocity, the time it takes for fully establishing a cavity is lower compared to the previous cases, and thus the initial curve at the formation phase is steeper than the case 3B. We observe almost a constant centerline film thickness after the cavity has been fully established. As expected the CFD solutions have predicted the thickness accurately when compared with the profile of the experiment. There are no cavity waves but a small fillet is observed right before the cavity fill-in. The size of this fillet is reduced when compared with the size of the fillet for the case 3B. This is also explained by the increased Re due to the higher velocity of the droplet. The 2D Fluent simulation predicts an earlier start of the retraction phase compared to 3D OpenFOAM and experiment. In this case, there is no splashing observed. The CFD solutions predict the formation of blobs on the crown that are formed in a similar manner as in the experiment. Both the CFD simulations predict a Worthington jet that leaves the top of the computational domain and that's where the track of centerline film thickness is lost, whereas in experiment the cavity fills in and reaches the initial height of static liquid layer quickly with some wobbling around that height. 


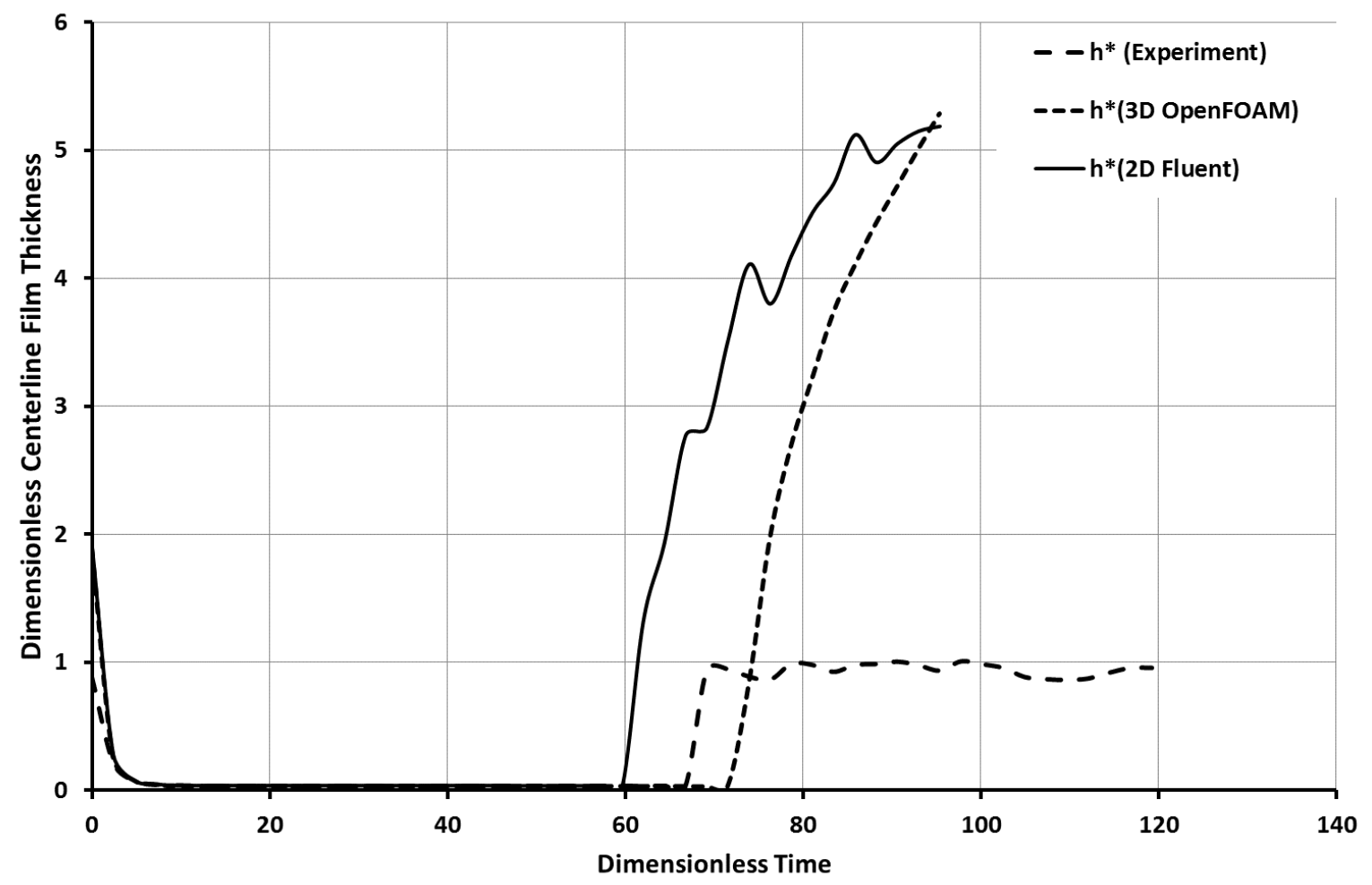

Figure 7.3-4: $h *$ vs $t *$ plot for case $5 \mathrm{~A}(\mathrm{We}=993, \operatorname{Re}=3574$, and $\mathrm{h} / \mathrm{d}=0.9)$.

Comparison of centerline film thickness time histories of CFD simulations and experiment for case 5C is showed in Figure 7.3-5. The velocity of the drop impacting the static liquid layer is 4.177 $\mathrm{m} / \mathrm{sec}$. Though the Re is less when compared to the previous case, splashing is observed in the CFD simulations. The reason behind this is fact that the static liquid layer is just $1 / 5^{\text {th }}$ the diameter of the drop. Despite the fact that the crown breaks up into drops in 3D OpenFOAM and rings in 2D Fluent simulations, CFD simulations predicted the centerline film thickness accurately after establishing the 


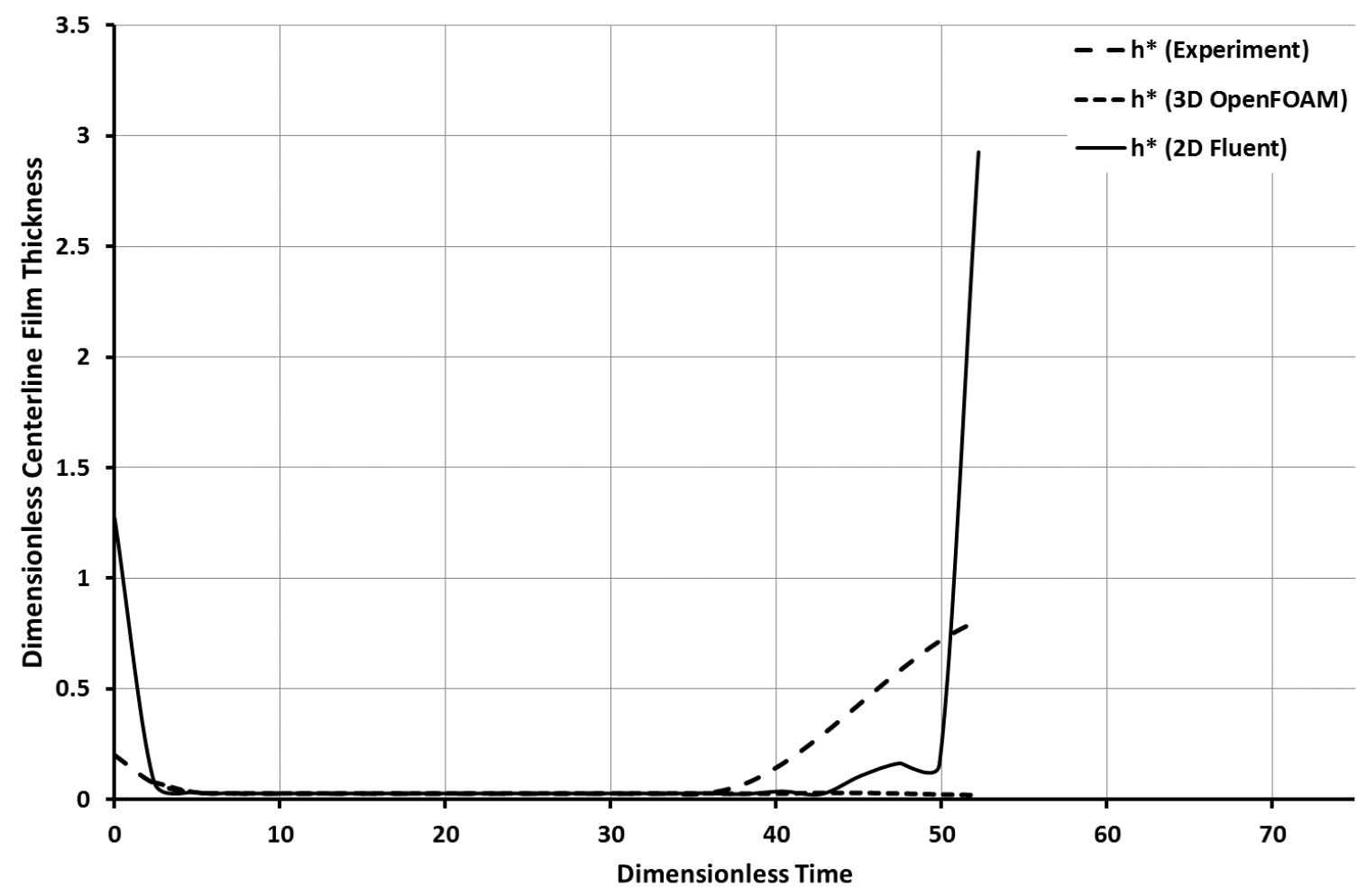

Figure 7.3-5: $h^{*}$ vs $t^{*}$ plot for case $5 \mathrm{C}(\mathrm{We}=984, \operatorname{Re}=3555$, and $\mathrm{h} / \mathrm{d}=0.2)$.

cavity fully. While the crown behaves like an elastic membrane in the experiment, in this case, there is no evidence of cavity waves. As there is lot of splashing involved in the 3D OpenFOAM simulation compared to 2D Fluent; most of the crown liquid has ejected away from the cavity in the 3D simulation and there is a loss of momentum that doesn't force the cavity to fill in as quickly as it should. Since the crown in the experiment breaks into droplets later than in either simulation, and thus acts like an elastic membrane for a longer time, all the liquid in the crown falls back into the center of the cavity, forcing it to fill in more quickly in experiment. This is the only case where the retraction phase of the experiment starts earlier than predicted by both of the CFD simulations.

\subsection{Comparison of Sub-Cavity Volumes}

The method developed for calculation of sub-cavity volumes has been discussed in Chapter 6 . Their importance in analyzing the heat transfer has also been discussed in Chapter 2. Here in this section of Chapter 7, the comparison of the normalized sub-cavity volumes as a function of non-dimensional time for both 2D axisymmetric Fluent cases and 3D OpenFOAM cases with the experimental results of Hillen (2013) is presented. The normalized sub-cavity volume is calculated by: 


$$
V^{*}=V_{\text {sub-cavity }} / V_{\text {drop }}
$$

The non-dimensional time is calculated as:

$$
t^{*}=t \frac{V e l}{d}
$$

In these comparisons, for two cases, i.e. for case $1 \mathrm{~A}$ and case $5 \mathrm{~A}$, the experimental sub-cavity volume calculations have been performed with Hillen's criterion (Hillen, 2013) applied to his experiment data of determining the sub-cavity cutoff height and calculating the sub-cavity volumes. In all five comparisons, calculations using two criteria are presented for the simulations. One is Hillen's criterion, while the other criterion, developed in the present work, has been described in Chapter 6 .

Figure 7.4-1 shows the comparison of normalized sub-cavity volumes as a function of normalized time for $2 \mathrm{D}$ and $3 \mathrm{D}$ simulations and experiment computed using different criteria for case $1 \mathrm{~A}$. This case has the thickest liquid film $\left(h^{*} \sim 1\right)$ with low We. Low We cases studied in this work do not splash. Velocity of the droplet impinging the static liquid layer is set to equate the Re obtained from sprays and its value is $1.727 \mathrm{~m} / \mathrm{sec}$. Because of this low Re in addition to the thick liquid film, most of the momentum generated due to the drop is spent in pushing the liquid away to form a cavity. The crown is barely formed. Because of the low momentum, the cavity formation is slowed down and hence the full establishment of the cavity is delayed. The cavity fill-in is also a gradual process and this can be observed with the gradual decrease in the dimensionless sub-cavity volume curve.

The rate at which the cavity expands is the same in all the simulations of this case, which agrees with the experiment in the initial stages. Maximum $\mathrm{V}^{*}$ occurs at $t^{*}=6.8$ for the experiment results presented in Hillen, 2013 and then a gradual decrease of the sub-cavity volume is observed for the rest of the cavity lifetime. When the same data is analyzed with running average criterion, then the maximum $\mathrm{V}^{*}$ occurs at $\mathrm{t}^{*}=5.7$ for the experiment. At the end of the cavity lifetime, the results obtained from Hillen, 2013 and the running average criterion follow the same trend. The experiment is performed in an environment that is not free from dust particles and temperature variations. 


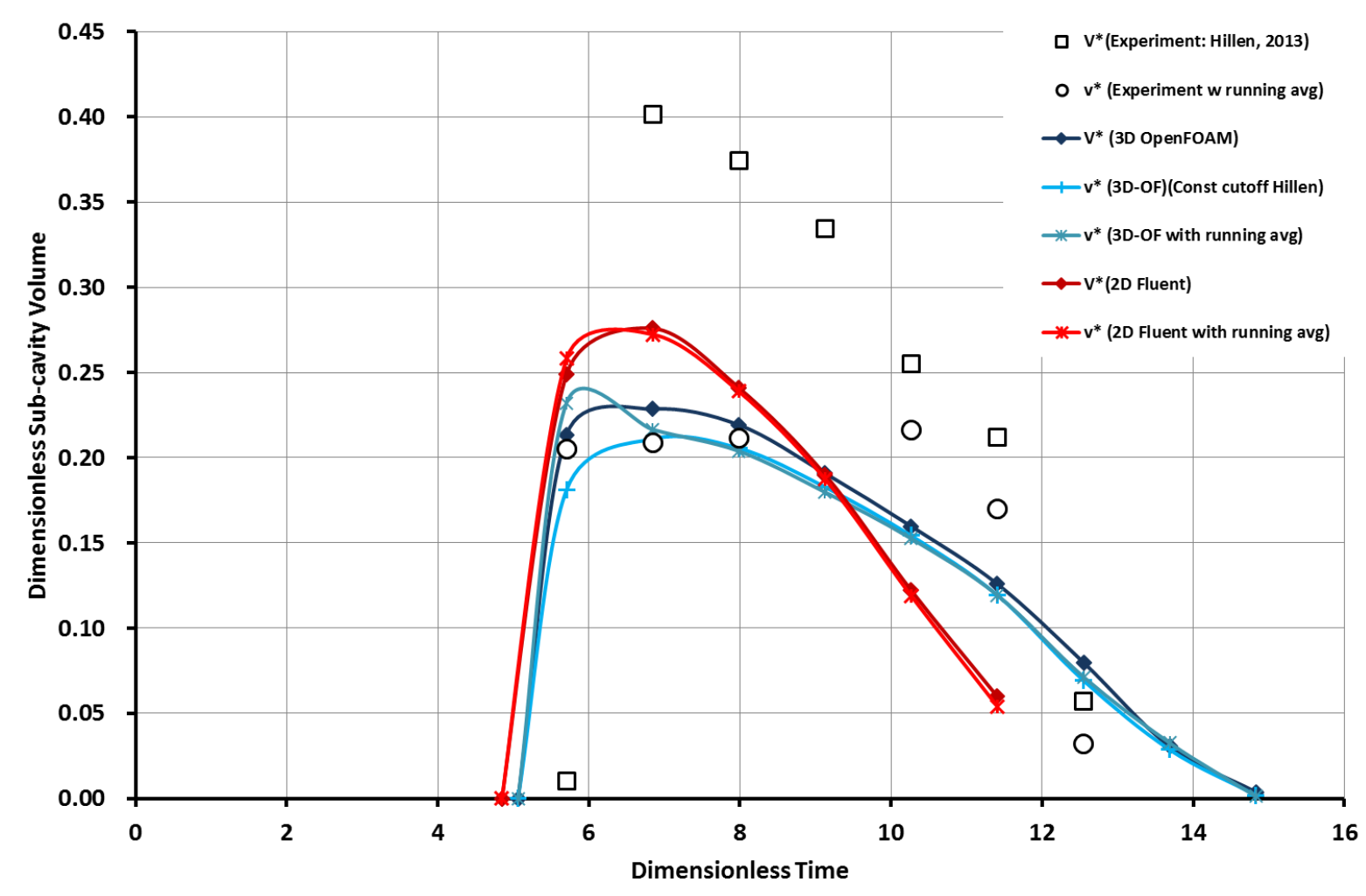

Figure 7.4-1: $V^{*}$ vs $t^{*}$ plot for case $1 \mathrm{~A}(\mathrm{We}=145, \operatorname{Re}=1189, \mathrm{~h} / \mathrm{d}=1.0)$.

Due to these reasons, the viscosity and surface tension are likely to change, whereas these values are kept constant in the CFD simulations at the values given in Table 5-1, and hence the cavity lifetimes are different. For 3D OpenFOAM simulation, the cavity lifetime is $42.7 \%$ higher than that of experiment's cavity lifetime and for 2D Fluent simulation it is $4.2 \%$ lower.

First, the sub-cavity volume comparison is made between volumes obtained from Hillen, 2013 and volumes obtained from the constant cut-off height criterion, followed by the comparison between the results obtained from the running average criterion. In the constant cut-off height criterion, the 3D OpenFOAM results, on an average, gave $27.6 \%$ less volume than the experiment whereas the 2D Fluent results gave $46.8 \%$ less volume. But when the criterion of comparison is changed to running average then the 3D OpenFOAM results gave $32.8 \%$ less volume than the experiment and 2D Fluent gave $48 \%$ less volume. For this case, the effect of which criterion is used in computing the sub-cavity volume is relatively small, but the 3D simulations are closer to the experimental results.

For 2D Fluent simulations, due to the lack of three dimensionality, the cavity lifetime is short and hence the sub-cavity volume as a function of time returns to zero too soon by about $\Delta t^{*} \sim 3-5$ for this 
case. The percentage of drop volume present in the sub-cavity volume predicted by 3D OpenFOAM is $23.2 \%$ and by $2 \mathrm{D}$ Fluent is $27.2 \%$ at maximum $\mathrm{V}^{*}$. Though the $2 \mathrm{D}$ Fluent gave a higher percentage prediction of drop volume, the cavity lifetime is shorter than that of the 3D OpenFOAM.

The normalized sub-cavity volumes calculated as a function of dimensionless time for both CFD simulations using two different criteria for the case 1C are shown in Figure 7.4-2. This is the lowest We case with the thinnest liquid film $\left(h^{*}=0.2\right)$. To perform the simulation with exactly the same Re computed for the experiment, the velocity of the droplet is set to $1.667 \mathrm{~m} / \mathrm{sec}$ at $\mathrm{t}=0$. Though the momentum of the droplet is less, it could create a cavity and form a crown. This crown collapses quickly and forces the cavity to fill-in by $t^{*} \sim 11-13$. Because of the thin liquid film, there is a steep rise in the sub-cavity volume in the initial stages of the impact and then the volume gradually decreases.

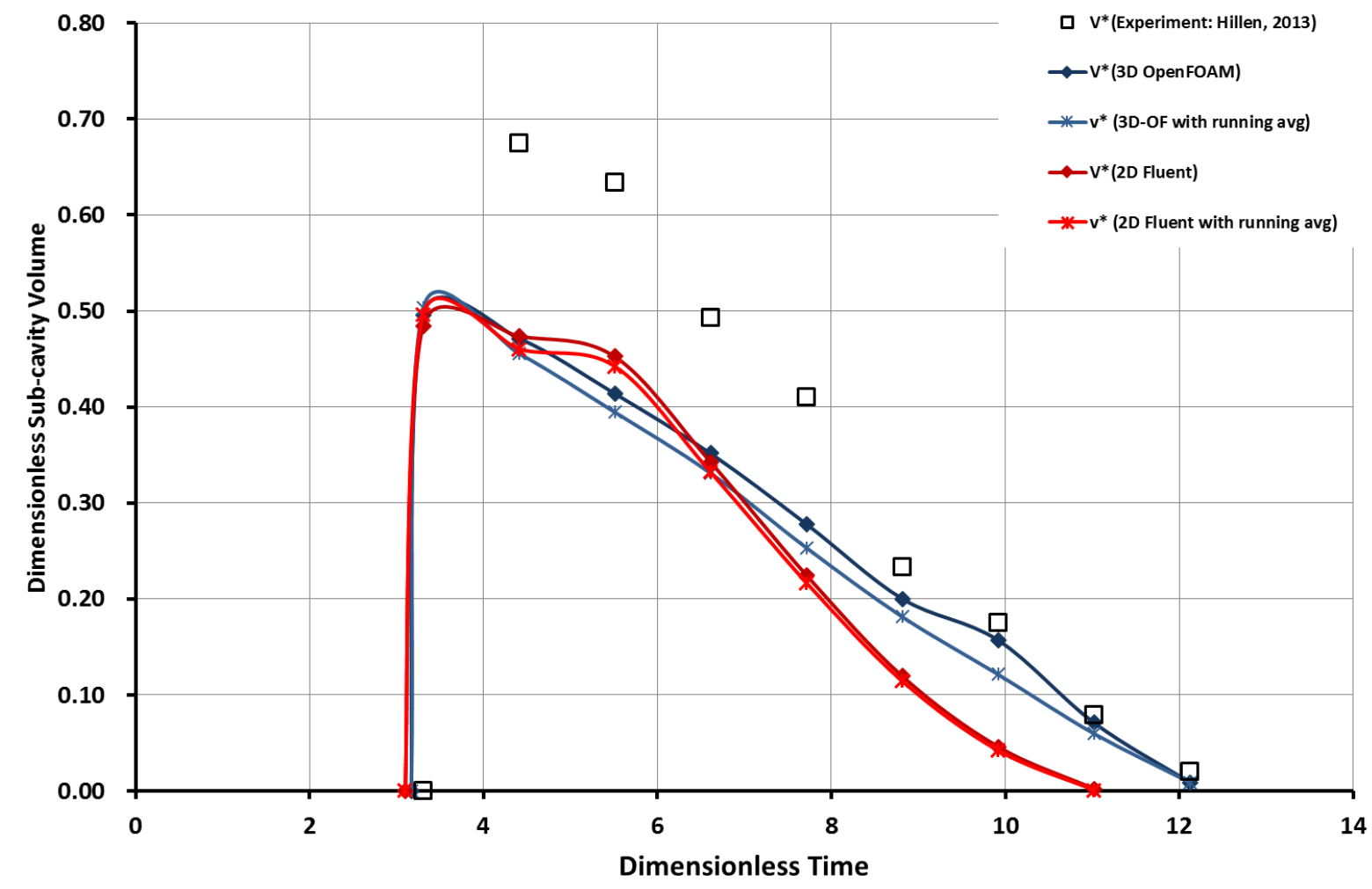

Figure 7.4-2: $V^{*}$ vs $t *$ plot for case $1 \mathrm{C}(\mathrm{We}=135, \mathrm{Re}=1176, \mathrm{~h} / \mathrm{d}=0.2)$.

The cavity formation begins early in the CFD simulations compared to the experiment but the end of the cavity is the same for 3D OpenFOAM and experiment. The 3D simulation can again predict the cavity lifetime more accurately than 2D Fluent for this case, which fills-in too quickly. The absence of 
three dimensionality in the 2D Fluent simulation, causes the cavity lifetime to be too short and the sub-cavity volume as a function of time to tend to zero sooner than 3D simulation and experiment by about $\Delta \mathrm{t}^{*} \sim 1-2$. The presence of dust particles in the experiment setup environment could cause the surface tension to decrease, offering less resistance when the cavity is expanding. This reason is speculated to be the cause of the higher sub-cavity-volume in the experiment than CFD simulations.

While the experiment shows the maximum $\mathrm{V}^{*}$ has occurred at $\mathrm{t}^{*}=4.4$, both the CFD simulations predicted a maximum $\mathrm{V}^{*}$ at $\mathrm{t}^{*}=3.3$. After the cavity starts retracting, the sub-cavity volume starts to fall almost linearly in both the experiment and 3D OpenFOAM solution. The 2D Fluent has a different curve shape and fills the cavity more quickly. The sub-cavity cavity lifetime predicted by the 2D Fluent simulation is about $10.2 \%$ less than that of the experiment's cavity lifetime whereas the 3D OpenFOAM predicted the cavity lifetime of $1.2 \%$ higher.

With the constant cutoff height criterion, the sub-cavity volume predicted by the $2 \mathrm{D}$ Fluent simulation, on an average, is $56.7 \%$ less than experiment's volume whereas the 3D OpenFOAM simulation predicted $27 \%$ less. When the sub-cavity volume is calculated using the running average criterion, the volume predicted is even less than the previous criterion. In this criterion, the 2D Fluent predicted $58.5 \%$ less volume and the 3D OpenFOAM simulation predicted $34.3 \%$ less than the experiment. All the CFD simulations predicted around 50\% of the drop volume is present in the subcavity volume, when it reaches its maximum value.

Figure 7.4-3 shows the comparison of normalized sub-cavity volumes as a function of normalized time for the 2D and 3D simulations and experiment computed using the two different criteria, discussed in previous chapters, for case 3B. The thickness of the liquid film is half of the diameter of the drop $\left(\mathrm{h}^{*} \sim 0.5\right)$ with a Weber number of 621 . This is an intermediate case between the highest We and lowest We cases discussed in this work. As seen in Figure 7.2-7, some secondary splashing is predicted in 3D OpenFOAM, which is caused by an inadequate mesh resolution in the region of the crown head. The velocity of the impacting droplet is $3.546 \mathrm{~m} / \mathrm{sec}$ at $\mathrm{t}=0$, which is considerably larger than the first two cases, and thus a larger cavity with increased cavity lifetime is observed. 


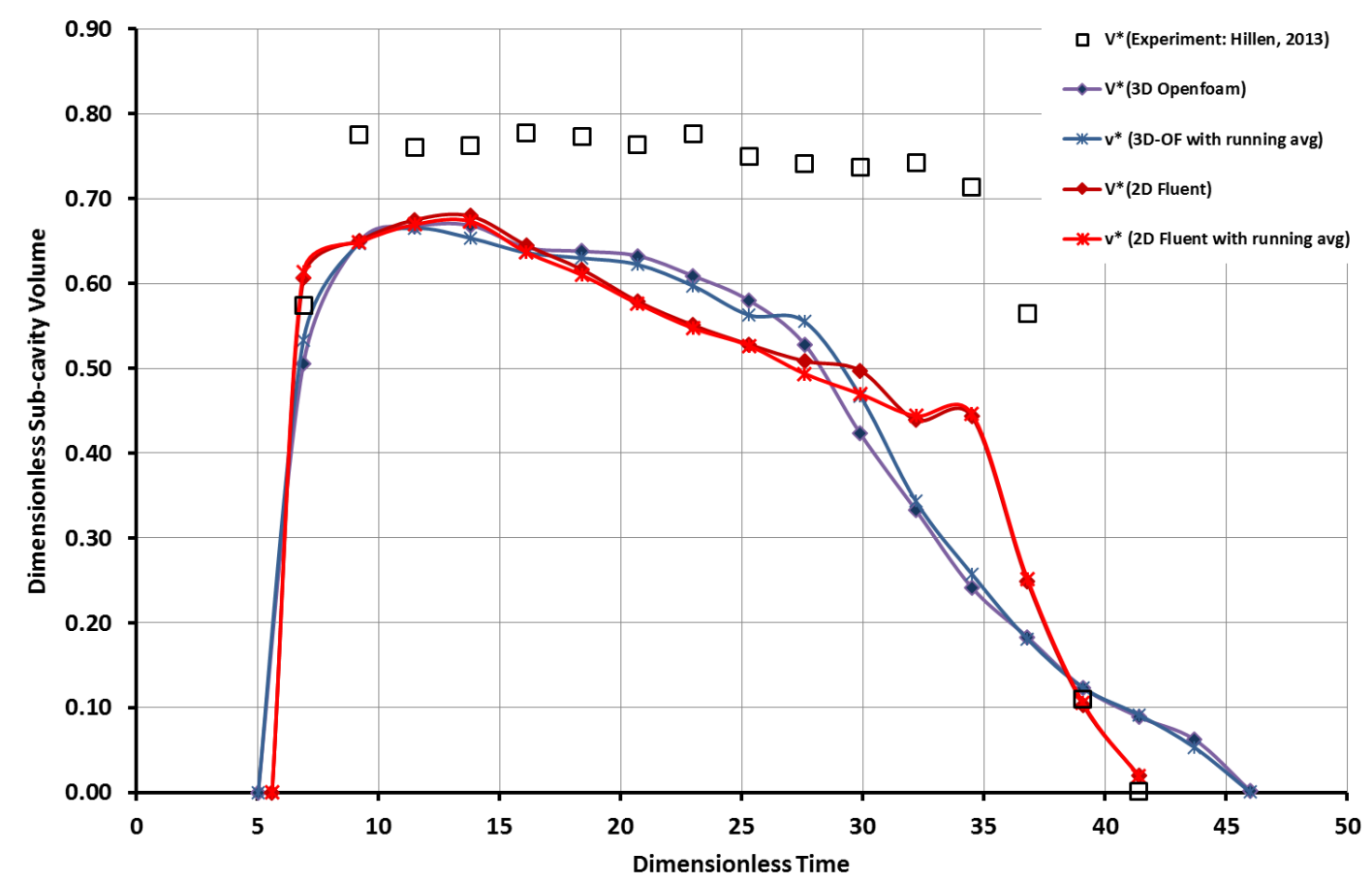

Figure 7.4-3: $V^{*}$ vs $t *$ plot for case 3B $(\mathrm{We}=621, \mathrm{Re}=2643$, and $\mathrm{h} / \mathrm{d}=0.5)$.

Despite the small delay in onset of the thin sub-cavity liquid volume predicted at the beginning of the 2D Fluent solution, all the simulations predicted the rate at which the cavity expands with reasonably good agreement to the experiment. Maximum sub-cavity volume is obtained at $t^{*}=13.7$ in all the CFD simulations, whereas in the experiment the maximum is at $t^{*}=9.2$ which is earlier than the CFD simulations. Sub-cavity volume remained nearly constant around the maximum value for the most of the cavity lifetime until it quickly fell to zero. There is a gradual decrease in the sub-cavity volume curve for 3D OpenFOAM simulation indicating that the cavity fill-in process is slow, whereas for the 2D Fluent simulation the cavity fill-in is quicker compared to 3D OpenFOAM, and the experiment has a steep descent in the curve. The difference in the cavity lifetime between 2D Fluent and 3D OpenFOAM is $\Delta \mathrm{t}^{*} \sim 3-4$. For the 3D OpenFOAM simulation, with the constant cutoff height criterion the sub-cavity volume predicted is $26.4 \%$ less than in the experiment on an average, and $25.9 \%$ less with the running average criterion when compared with the experiment. The $2 \mathrm{D}$ Fluent simulation with the constant cutoff height criterion predicted $23.8 \%$ less sub-cavity volume, while with the running average criterion it is $24.2 \%$ less than the experiment value.

After discussing the sub-cavity volume, the amount of drop liquid present in the sub-cavity is presented. The experiment showed that there is $77.7 \%$ of drop liquid present in the sub-cavity volume 
whereas the 3D OpenFOAM simulation predicted that $66.8 \%$ and 2D Fluent predicted $68 \%$ is present. The greater the percentage of drop liquid present in the sub-cavity volume, the more the local heat flux would be expected to increase, which would decrease the temperature of the heated surface.

In Figure 7.4-4, the normalized sub-cavity volumes computed for the 2D and 3D CFD simulations using two different criteria are compared with the experimental calculations for case 5A. This case has the highest Weber number and the thickest liquid film $\left(\mathrm{h}^{*} \sim 1\right)$. To match the Reynolds number of the impinging droplet with that of the Reynolds number derived from the sprays, the velocity of the droplet is $4.196 \mathrm{~m} / \mathrm{sec}$ at $\mathrm{t}=0$. Due to this velocity, the drop impacts the static liquid with large momentum and forms a cavity with even larger radius. Because of the momentum of the drop, there is also a steep rise in the $V^{*}$ curve in the initial stages of impact. This is also the primary reason for the longest cavity lifetime compared to the other cases.

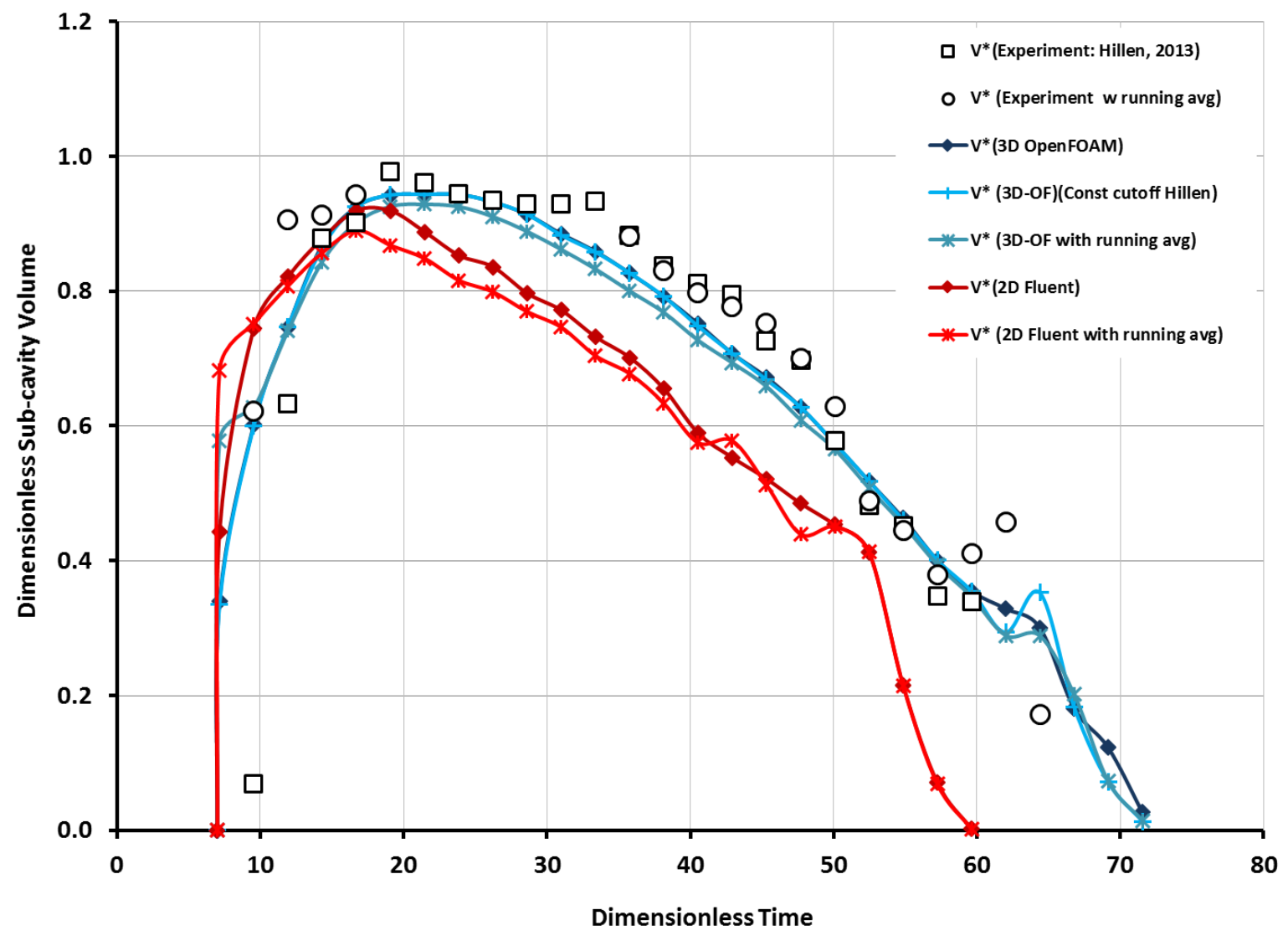

Figure 7.4-4: $V^{*}$ vs $t *$ plot for case $5 \mathrm{~A}(\mathrm{We}=993, \mathrm{Re}=3574$, and $\mathrm{h} / \mathrm{d}=0.9)$.

During the initial stages of sub-cavity volume calculations, all the simulations had good agreement of the volumes during the cavity formation phase with the experimental results. It can be observed that 
the maximum $\mathrm{V}^{*}$ occurred at $\mathrm{t}^{*}=19.1$ for the experiment and then a gradual decrease is observed towards the end of the cavity lifetime. The same trend of first sub-cavity volume calculation, reaching the maximum volume at $t^{*}=19.1$ and the decrease in volume towards the end is also seen in the subcavity volume calculations of 2D and 3D simulations. The presence of minute dust particles around the experiment setup and changes in the surrounding air temperature would cause the viscosity and surface tension to change. In the simulations, these values remain constant. This could be the cause of the observed differences in cavity lifetime. The sub-cavity lifetime in 3D OpenFOAM is $17.7 \%$ higher than the experimental cavity lifetime and in 2D Fluent it is $4.1 \%$ lower.

From the plot, it is seen that the $\mathrm{V}^{*}$ profiles obtained from the two criteria nearly overlap and gave essentially the same results. When the sub-cavity volume comparison is made between the two criteria against the experimental results, it is observed that, on average the sub-cavity volume obtained from 3D simulations are $2.7 \%$ high relative to the experimental results by running average criterion, and $6.2 \%$ high by the constant cutoff height criterion. For the 2D simulations, a decrease in the sub-cavity volume of $25.1 \%$ is obtained by the running average criterion compared to the experiment, and a $22.9 \%$ decrease is obtained using the constant cutoff height criterion.

Because of the absence of three dimensionality in the $2 \mathrm{D}$ simulations, the cavity lifetime is too short and this results in sub-cavity volume as a function of time tending to zero too soon by about $\Delta \mathrm{t}^{*} \sim 10$ 12 for this case. The volume of the sub-cavity liquid is predicted in the 3D simulations to be $92.6 \%$ drop liquid. When a cold drop impacts the heated liquid layer, due to the temperature difference between the heated liquid in the layer and the drop liquid, there is expected to be an increased heat flux at that location. Thus, the high We and Re droplet impacts can more effectively extract the heat from the surface, both due to a greater percentage of drop liquid in the sub-cavity volume, and due to the increased cavity lifetime.

Case $5 \mathrm{C}$ is different from the above cases and has high We and also high Re. Figure 7.4-5 shows the normalized sub-cavity volume curve as a function of normalized time for case $5 \mathrm{C}$. In addition to these high Re and We values, the thin liquid film is $1 / 5^{\text {th }}$ the drop diameter making it a shallow liquid film. To match the Re derived from the sprays, the velocity of the impinging droplet is set as $4.177 \mathrm{~m} / \mathrm{sec}$ at 
$\mathrm{t}=0$. Due to the high momentum imparted to the thin liquid film, there are a lot of secondary droplets ejected out of the crown. This splashing makes it very difficult for the CFD simulations to match the cavity lifetime and the sub-cavity volumes measured in the experiment.

As discussed earlier, the crown in this case acts like an elastic membrane so long as it is intact, which first expands due to the momentum of the drop, and then contracts and collapses to the center of the cavity due to surface tension. The cavity lifetime is greatly affected by this type of behavior. Note that the $2 \mathrm{D}$ and $3 \mathrm{D}$ simulations both predict that the entire crown breaks up into secondary drops much earlier than is observed in the experiments (Figure 7.2-9). Thus, the cavity lifetime is much longer for both the simulations than the experiment (Figure 7.3-5). The cavity of the experiment fills-in quickly by about $\Delta \mathrm{t}^{*}=19$ when compared with the 3D OpenFOAM simulation and by $\Delta \mathrm{t}^{*} \sim 7$ for the $2 \mathrm{D}$ Fluent simulation.

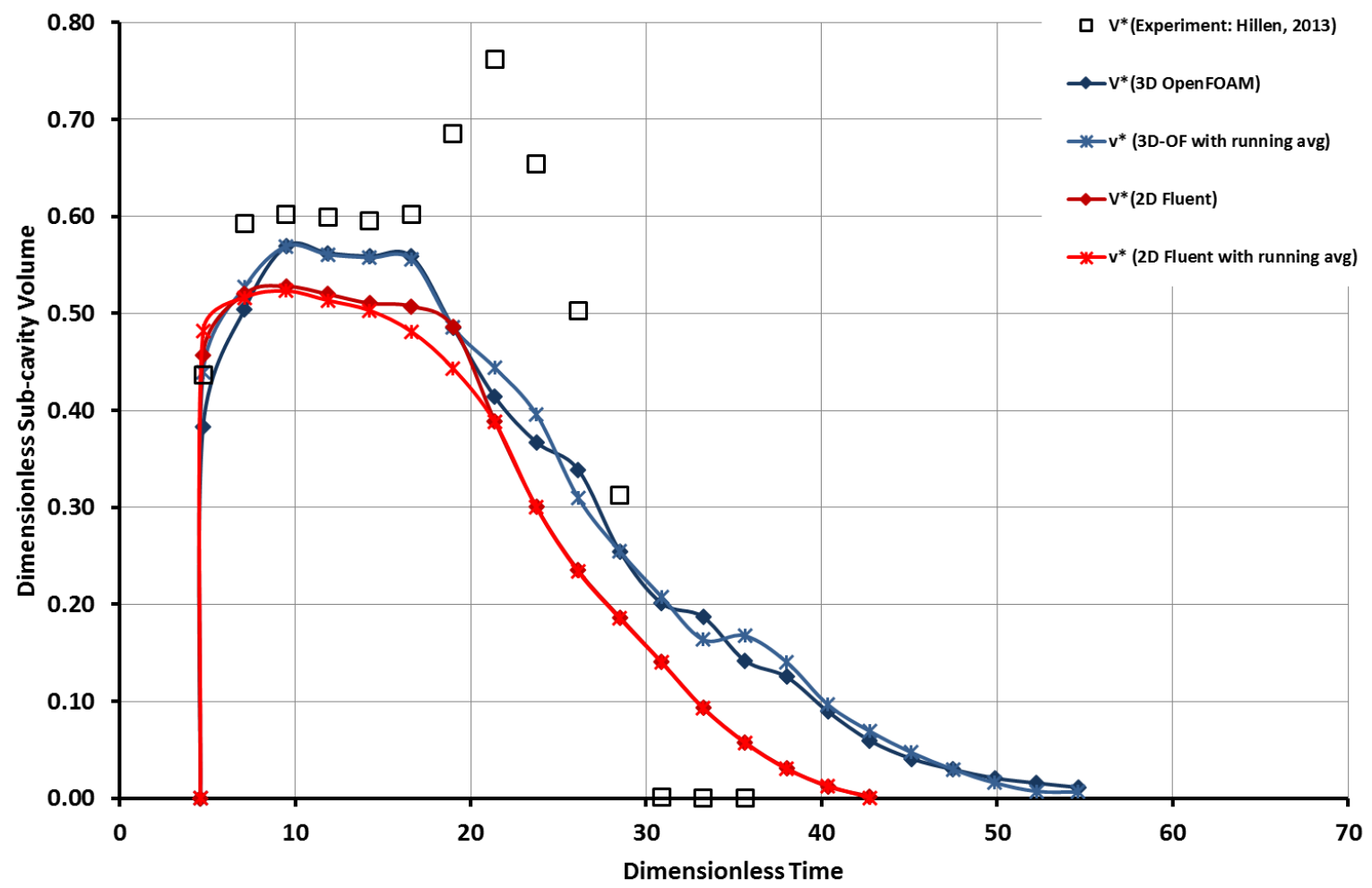

Figure 7.4-5: $v^{*}$ vs $t *$ plot for case $5 \mathrm{C}(\mathrm{We}=984, \operatorname{Re}=3555$, and $\mathrm{h} / \mathrm{d}=0.2)$.

The experiment shows that the maximum sub-cavity volume is achieved at $t^{*}=23.7$ which is near to the end of the cavity lifetime, while the CFD simulations here predicted a maximum volume at $\mathrm{t}^{*}=$ 9.5. The maximum volume obtained by the experiment at the end of the cavity is believed not to be 
realistic. This may be due to some undiagnosed limitations of the equipment that was used to capture the cavity during the crown collapse. Or, perhaps there was interaction of the capillary or cavity wave that caused this increase in the sub-cavity volume late in its lifetime, but only for this case.

The sub-cavity volume was again calculated with two different criteria. With the constant cutoff height criterion, the 2D Fluent simulation on an average has predicted the sub-cavity volume of $31 \%$ less than that of experiment, whereas the 3D OpenFOAM simulation predicted a value $22.8 \%$ less. By the running average criterion on the other hand, the 2D Fluent simulation has predicted $32.4 \%$ less than the experiment and the 3D OpenFOAM simulation predicted $22 \%$ less. In Table (7.3-7-2, there is seen to be an average of $76.2 \%$ of the drop volume present in the sub-cavity volume as observed in the experiment, $57 \%$ of the drop volume as predicted by the 3D OpenFOAM simulation, and $52.3 \%$ is predicted by the 2D Fluent simulation.

Table 7-1: Percentage deviation of sub-cavity volume for all the cases.

\begin{tabular}{|c|c|c|c|c|}
\hline Percentage Deviation & \multicolumn{2}{|c|}{ 2D Fluent and Experiment } & \multicolumn{2}{c|}{ 3D Fluent and Experiment } \\
\hline Cases/Criteria & $\begin{array}{c}\text { Constant } \\
\text { Cutoff }\end{array}$ & $\begin{array}{c}\text { Running } \\
\text { Average }\end{array}$ & $\begin{array}{c}\text { Constant } \\
\text { Cutoff }\end{array}$ & $\begin{array}{c}\text { Running } \\
\text { Average }\end{array}$ \\
\hline $\mathbf{1 A}$ & -46.8 & -48 & -27.6 & -32.8 \\
\hline $\mathbf{1 C}$ & -56.7 & -58.5 & -27 & -34.3 \\
\hline $\mathbf{3 B}$ & -23.8 & -24.2 & -26.4 & -25.9 \\
\hline $\mathbf{5 A}$ & -22.9 & -25.1 & 6.2 & 2.7 \\
\hline $\mathbf{5 C}$ & -31 & -32.4 & -22.8 & -22 \\
\hline
\end{tabular}

Table 7-2: Percentage of drop liquid volume present in the sub-cavity volume.

\begin{tabular}{|c|c|c|}
\hline Case/CFD & 2D Fluent & 3D OpenFOAM \\
\hline $\mathbf{1 A}$ & 27.2 & 23.2 \\
\hline $\mathbf{1 C}$ & 50 & 50 \\
\hline 3B & 68 & 66.8 \\
\hline $\mathbf{5 A}$ & 92.6 & 94.2 \\
\hline $\mathbf{5 C}$ & 52.3 & 57 \\
\hline
\end{tabular}




\section{Conclusions and Future Work}

\subsection{Conclusions}

After discussing the brief history and the applications of spray cooling, it was concluded to be essential to study and improve the present spray cooling methods to achieve higher heat fluxes without reaching critical heat flux. Sprays consist of millions of droplets and one drop among them was taken for studying the properties of the sub-cavity region. By varying the non-dimensional parameters (We, Re and $\mathrm{h}^{*}$ ) of the liquid, the single drop impacts studied previously through experiments (Hillen, 2013) have been modeled using two different CFD simulation methods for comparisons with the experimental results.

In this work comparison is made between the experimental results and the CFD simulations so as to know the accuracy of the predictions made by the CFD simulations. The results obtained from the CFD simulations are discussed in Chapter 7. Section 7.2 shows the side by side image comparisons of different cases obtained from the Hillen 2013 thesis and the CFD simulations. In a general perspective, the 3D OpenFOAM simulations of lower We cases gave a good agreement with the experiments. For the lower We case with the thickest liquid film, the 3D OpenFOAM results predicted a higher cavity lifetime than the $2 \mathrm{D}$ Fluent simulation but for the same low We case with thin liquid film, the 3D OpenFOAM simulation predicted the cavity lifetime accurately. Though the experiments do not show any secondary splashing for the case 3B, the crown of the 3D OpenFOAM simulation breaks. This is believed to be due to the insufficient mesh refinement near the crown. In this case, the 2D Fluent simulation predicted accurately due to the fine mesh. It is expected that, with the refined mesh, the 3D OpenFOAM simulation could also predict accurately.

Of all the cases, case 5C with high We and Re, and thin liquid film is different. As a result of the high Re combined with high We, there is a lot of splashing observed in both CFD simulations and experiment, but in the experiment the crown remains intact longer and acts like an elastic membrane because of surface tension, whereas in CFD simulations it is believed that due to a lack of mesh refinement the crown breaks into secondary splashing in 3D OpenFOAM and rings in 2D Fluent simulations. This leads to an overprediction of the cavity lifetime. 
In section 7.3 , the centerline film thickness time history comparisons of all five cases between experiments and both CFD simulations has been discussed. For all five cases the early-time simulations, and the predicted minimum film thickness values agree well with experiments. However, for all five cases, both the 2D and 3D simulations do not predict the retraction phase well. The best agreement is observed for the lowest We cases $1 \mathrm{~A}$ and $1 \mathrm{C}$, and the worst agreement is observed for the highest-We, thinnest $h^{*}$ case 5C. It is believed that adaptive meshing would be necessary in the 3D OpenFOAM simulations in order to achieve better simulation results.

In Section 7.4, the percentage of agreement achieved by the CFD simulations with the experiments in predicting the sub-cavity volumes has been discussed. Though the CFD simulations of low We cases agree well with the cavity shape and cavity lifetime of the experiments, the sub-cavity volumes do not agree well. As the We increases, the sub-cavity volumes agree reasonably well with the experiments. The best and worst agreement is seen in high We and high Re cases. The CFD simulations and experiments both agree with the percentage of drop volume present in the sub-cavity volume for case 5A. Comparing between 2D Fluent and 3D OpenFOAM simulations, 3D OpenFOAM generally gives a more accurate prediction. However, both the $2 \mathrm{D}$ and $3 \mathrm{D}$ case $5 \mathrm{C}$ simulations give a bad prediction of cavity lifetime, the behavior of the crown, and also percentage of drop volume present in the subcavity volume.

The percentage of drop volume present in the sub-cavity volume is the important factor to be studied for achieving the effective cooling of the heated surface. The higher the drop volume is present in the sub-cavity volume, the higher will be the temperature difference between the hot surface and the spray liquid. Because of this difference, there is a higher heat transfer coefficient obtained. High Re with high We and thick liquid film gives longer cavity lifetime and higher percentage of drop volume in the sub-cavity volume.

\subsection{Future Work}

The present work is helpful in predicting the type of liquid and spray conditions to be used for the future study of sprays. More accurate predictions should be made with different domains such as a cylindrical domain in 3D simulations, to eliminate the problems that rise at the diagonal corners of the 
mesh. With higher computational power, the mesh can be refined to some extent, especially by using an adaptive mesh, the simulations can be improved to predict more accurate results. Also, the present simulations should be re-analyzed in order to compute the percentages of the sub-cavity volumes that originate from the drop liquid, and from the liquid layer.

In future simulations, the energy equation should be added to simulate the temperature variations of drop liquid and film liquid, thus predicting the heat transfer rates, while also including the buoyancy effects. With a heated surface, and imposing the nucleate boiling and evaporation models into the simulation, the heat flux and the heat transfer coefficient can be studied at the center of the cavity. Combining the effects of temperature variations and heated surface, the actual prediction of heat transfer rates can then be made. This is felt to be the most critical work yet to be undertaken. 


\section{References}

ANSYS Fluent 6.3 User's Guide - 23.9.4 Including Body Forces, 2006, Web, 13 Jan. 2015. $<$ http://aerojet.engr.ucdavis.edu/fluenthelp/html/ug/node929.htm>.

ANSYS Fluent 6.3 User's Guide - 25.3.3 Evaluation of Gradients and Derivatives, 2006, Web, 14 Jan. 2015. <http://aerojet.engr.ucdavis.edu/fluenthelp/html/ug/node994.htm>.

ANSYS Fluent 6.3 User's Guide - 25.4.3 Pressure-Velocity Coupling, 2006, Web, 13 Jan. 2015. <http://aerojet.engr.ucdavis.edu/fluenthelp/html/ug/node998.htm\#eq-simple-pc>.

Berberovic, E., van Hinsberg, N. P., Jakirlic, S., Roisman, I. V., and Tropea, C., "Drop impact onto a liquid layer of finite thickness: Dynamics of the cavity evolution," Physical Review, The American Physics Society, 79, 036306-1-9, 2009.

Brackbill, J. U., Kothe, D. B., and Zemach, C., "A continuum method for modeling surface tension." Journal of Computational Physics, 100.2, 335-54, 1992.

Campbell, N. A. F., Hawley, J. G., Leathard, M. J., Horrocks, R. W., and Wong, L., "Nucleate boiling investigations and the effects of surface roughness", Warrendale, PA: SAE International, 1999.

Caraba, E., "Preconditioned conjugate gradient algorithm," Louisiana State University, 2008.

EnSight, Web, 4 Jan. 2015. <https://www.ceisoftware.com/>.

Gehring, E., Soriano, G., Lin, Y. P., Alvarado, J. L., and Trujillo, M. F., "Film dynamics relevant to spray cooling," 11th International Conference on Advanced Computational Methods and Experimental Measurements in Heat Transfer, July, 2010.

Hillen, N. L., Kuhlman, J. M., Dinc, M., and Gray, D. D., "Drop impingement on wet and dry surfaces," Paper AIAA-2012-2960, AIAA 42nd Fluid Dynamics Conference, New Orleans, LA, June 25-28, 2012.

Hillen, Nicholas L., "Droplet impact sub-cavity histories and PDPA spray experiments for spray cooling modeling", Master's thesis, West Virginia University, 2013.

Hirt, C. W., and Nichols, B. D., "Volume of fluid (VOF) method for the dynamics of free boundaries," Journal of Computational Physics, 39.1, 201-25, Web, 1981, <http://www.sciencedirect.com/science/article/pii/0021999181901455>.

Hussain, M., Peglow, M., Tsotsas, E., and Kumar, J., "Modeling of aggregation kernel using Monte Carlo simulations of spray fluidized bed agglomeration," AIChE J., 60.3, 855-68, 2014.

Issa, R.I., "Solution of the implicitly discretised fluid flow equations by operator-splitting," Journal of Computational Physics, 62.1, 40-65, 1986. 
Jaridi, M., Kuhlman, J. M., and Gray, D. D., "Spray cooling heat transfer mechanisms", Final Report for NASA EPSCoR Award No. NNX10AN04A, Oct., 2014.

Jasak, H., Jemcov, A., and Tuković, Ž., "OpenFOAM: A C++ library for complex physics simulations", International Workshop on Coupled Methods in Numerical Dynamics, 47-66, 2007.

Josserand, C., and Zaleski S., "Droplet splashing on a thin liquid film", Physics of Fluids, 15.6, 165057, 2003.

Josserand, C., Ray P., and Zaleski S., "Air entrapment and splashing treshold in drop impacts", $7^{\text {th }}$ International Conference on Multiphase Flow, International Conference on Multiphase Flow (ICMF), 55, 2010.

Kreitzer, P. J., "Spray cooling simulation implementing time scale analysis and Monte Carlo method", Ph.D. Dissertation, West Virginia University, Morgantown, WV, 2010.

Kuhlman, J. M., Kreitzer, P. J., Mehra, D., Gray, D. D., and Yerkes, K. L., "Influence of the Coulomb force on spray cooling," STAIF Conference on Thermophysics Applications in Microgravity, edited by M. El-Genk, AIP Conference Proceedings 504, New York, 2007.

Kuhlman, J. M., Gray, D. D., and Kreitzer P. J., "Progress in modeling of spray cooling," Proceedings of the 24th European Conference on Liquid Atomization and Spray Systems(ILASS), 7 pages, Estoril, Portugal, September 5-6, 2011. Poster presented at the European Conference on Liquid Atomization and Spray Systems (ILASS), Estoril, Portugal, September 5-7, 2011.

Kuhlman, J. M., Hillen, N. L., Dinc, M., and Gray, D. D., "Liquid volume measurements in the cavity formed by single droplet impacts into a thin, static liquid film", Experimental Thermal and Fluid Science, 54, 179-88, 2014.

NSA - Supercomputing Research, 2009, Web, 27 Jan. 2015. <https://www.nitrd.gov/pubs/implementation/1997/98.html>.

Sarkar, S., and Selvam. R. P., "Direct numerical simulation of heat transfer in spray cooling through 3D multiphase flow modeling using parallel computing," J. Heat Transfer, 131.12, 121007-1 to 121007-8, 2009.

Silk, Eric A., Eric L. G., and Selvam, R. P., "Spray cooling heat transfer: Technology overview and assessment of future challenges for micro-gravity application," Energy Conversion and Management, 49.3, 453-68, 2008.

Yarin, A. L., "Drop impact dynamics: Splashing, spreading, receding, bouncing...", Annual Review of Fluid Mechanics, 38.1, 159-92, 2006.

Youngs, D. L., "Time-dependent multi-material flow with large fluid distortion," Numerical methods for fluid dynamics, 24, 273-85, 1982. 